\title{
Review \\ Interdisciplinary Care Networks in Rehabilitation Care for Patients with Chronic Musculoskeletal Pain: A Systematic Review
}

\author{
Cynthia Lamper ${ }^{1, * \mathbb{C}}$, Laura Beckers ${ }^{1}$, Mariëlle Kroese ${ }^{2} \mathbb{D}$, Jeanine Verbunt ${ }^{1,3}$ and Ivan Huijnen ${ }^{1,3}$ (i) \\ 1 Department of Rehabilitation Medicine, Functioning, Participation \& Rehabilitation, Care and Public Health \\ Research Institute (CAPHRI), Faculty of Health, Medicine and Life Sciences, Maastricht University, \\ 6229 ER Maastricht, The Netherlands; laura.beckers@maastrichtuniversity.nl (L.B.); \\ jeanine.verbunt@maastrichtuniversity.nl (J.V.); ivan.huijnen@maastrichtuniversity.nl (I.H.) \\ 2 Department of Health Services Research, Care and Public Health Research Institute (CAPHRI), \\ Faculty of Health, Medicine and Life Sciences, Maastricht University, 6229 ER Maastricht, The Netherlands; \\ marielle.kroese@maastrichtuniversity.nl \\ 3 Centre of Expertise in Rehabilitation and Audiology, Adelante, 6432 CC Hoensbroek, The Netherlands \\ * Correspondence: cynthia.lamper@maastrichtuniversity.nl
}

Citation: Lamper, C.; Beckers, L.; Kroese, M.; Verbunt, J.; Huijnen, I. Interdisciplinary Care Networks in Rehabilitation Care for Patients with Chronic Musculoskeletal Pain: A Systematic Review. J. Clin. Med. 2021, 10, 2041. https://doi.org/10.3390/ jcm10092041

Academic Editor: Kelly Ickmans

Received: 29 March 2021

Accepted: 3 May 2021

Published: 10 May 2021

Publisher's Note: MDPI stays neutral with regard to jurisdictional claims in published maps and institutional affiliations.

Copyright: (C) 2021 by the authors. Licensee MDPI, Basel, Switzerland. This article is an open access article distributed under the terms and conditions of the Creative Commons Attribution (CC BY) license (https:// creativecommons.org/licenses/by/ $4.0 /)$.

\begin{abstract}
This systematic review aims to identify what rehabilitation care networks, within primary care or between primary and other health care settings, have been described for patients with chronic musculoskeletal pain, and what their impact is on the Quadruple Aim outcomes (health; health care costs; quality of care experienced by patients; work satisfaction for health care professionals). Studies published between 1 January 1994 and 11 April 2019 were identified in PubMed, CINAHL, Web of Science, and PsycInfo. Forty-nine articles represented 34 interventions: 21 within primary care; 6 between primary and secondary/tertiary care; 1 in primary care and between primary and secondary/tertiary care; 2 between primary and social care; 2 between primary, secondary/tertiary, and social care; and 2 between primary and community care. Results on impact were presented in 19 randomized trials, 12 non-randomized studies, and seven qualitative studies. In conclusion, there is a wide variety of content, collaboration, and evaluation methods of interventions. It seems that patient-centered interdisciplinary interventions are more effective than usual care. Further initiatives should be performed for interdisciplinary interventions within and across health care settings and evaluated with mixed methods on all Quadruple Aim outcomes.
\end{abstract}

Keywords: interdisciplinary care; rehabilitation care; care networks; Quadruple Aim; health; experienced quality of care; satisfaction with work; cost-effectiveness; primary care

\section{Introduction}

Chronic musculoskeletal pain (CMP) is a leading cause of disability occurring in $19-28 \%$ of the European population $[1,2]$. As many as one-third of primary care consultations concern CMP complaints such as back or knee pain [3]. Complaints often persist for more than five years and have significant impacts on patients' daily life, leading to high societal and health care costs [4-6].

Research indicates that pain needs an integrated biopsychosocial approach to decrease its impact on health. Nevertheless, this impact is expected to increase as people live longer [7,8]. Current care is organized in "silos" with a focus on only one aspect of pain (biomedical, psychological, or societal), instead of an integrated approach. There is little coordination and communication among health care professionals (HCPs), leading to fragmented care [9-11]. This results in many monodisciplinary treatments, with a wide variety of treatment approaches, restricted in available time and resources. Thus, there is a call for a different organization of care for patients with CMP [12]. As a possible 
solution for this fragmented care, the general practitioner (GP) should have a more prominent role in managing patients with chronic and complex diseases such as CMP [13], as there is a need for continuity, comprehensiveness, and coordination in CMP care [14]. Primary care should play a central role in effectively managing and integrating care with mono- and multidisciplinary treatments, with the GP as the case-manager for chronic and complex diseases [15].

Accordingly, the World Health Organization (WHO) developed a guideline for redesigning rehabilitation in health systems [16]. It indicates that rehabilitation services should be integrated within primary care, as well as between primary, secondary, and tertiary levels of health systems, with a case-management role for primary care. This is in line with Coleman et al., who state that disease management interventions that target only patients may be less effective than those that also focus on organization of care and redesign of care delivery [9]. Cieza et al. advise scaling up rehabilitation services in primary care worldwide to ensure that a life-course and integrated perspective on care is achieved [2]. CMP must be approached within a biopsychosocial framework in order to deliver the most effective treatment. Depending on the complexity of the pain problem, it must be provided by different health care disciplines in collaborative teams, either in primary care alone or combined with secondary and tertiary care $[17,18]$.

Collaborative teams of HCPs for pain management in primary care could range in scope from less extensive combinations of GPs and HCPs, focusing on physical and psychological aspects of pain, to broad teams including rehabilitation, psychology, nursing, and case management $[19,20]$. If these treatments are multimodal, meaning one therapeutic aim per discipline, and involve HCPs from different disciplines, they are termed "multidisciplinary" [21]. For patients with more complex complaints, an interdisciplinary treatment is needed, where all HCPs involved have a common therapeutic aim and a shared biopsychosocial focus. These interdisciplinary care networks can improve clinical care and service delivery, as suggested by Coleman et al. [9].

In order to optimize health system performance, including interdisciplinary care networks, an approach known as the Quadruple Aim is recommended. This comprises four dimensions: health, quality of care experienced by patients, healthcare costs, and HCP work satisfaction $[22,23]$. There is some evidence that interdisciplinary care networks designed for various diseases can improve these four Quadruple Aim pillars [24-26]. Moreover, interdisciplinary care networks for CMP, incorporating a biopsychosocial model in assessing and treating pain, can result in pain reduction, improved quality of life, and improved social functioning [20]. In some cases, return-to-work and vocational outcomes may be seen.

However, it is not known which biopsychosocial interdisciplinary care networks exist within primary care, or between primary care and other health care settings, for the rehabilitation of patients with CMP. Furthermore, the impact of such networks on the Quadruple Aim outcomes of health, health care costs, quality of care experienced by patients, and work satisfaction of HCPs, is unknown. Therefore, this study aims to address these uncertainties. The first research question is: Which interdisciplinary care networks within primary care and between primary care and other health care settings have been implemented in rehabilitation care for patients $\geq 18$ years with CMP over the last 25 years? The second question is: What is the impact of these interdisciplinary care networks on rehabilitation care for patients with CMP, in terms of the Quadruple Aim outcomes: health, health care costs, quality of care experienced by patients, and HCP work satisfaction?

\section{Materials and Methods}

We conducted a systematic literature review to synthesize studies with interdisciplinary care networks in care for patients with CMP. These interdisciplinary care networks must be implemented within primary care or between primary care and other healthcare settings (secondary or tertiary care, social care, or community-based care) (see Figure 1). As this research did not involve human subjects, we did not seek ethics clearance for 
the project. The protocol was registered in the international prospective register of systematic reviews (PROSPERO; http:/ / www.crd.york.ac.uk/PROSPERO/ (accessed date: 6 May 2021)) on 28 August 2020 (registration number CRD42020158057). The review was conducted following PRISMA guidelines $[27,28]$.

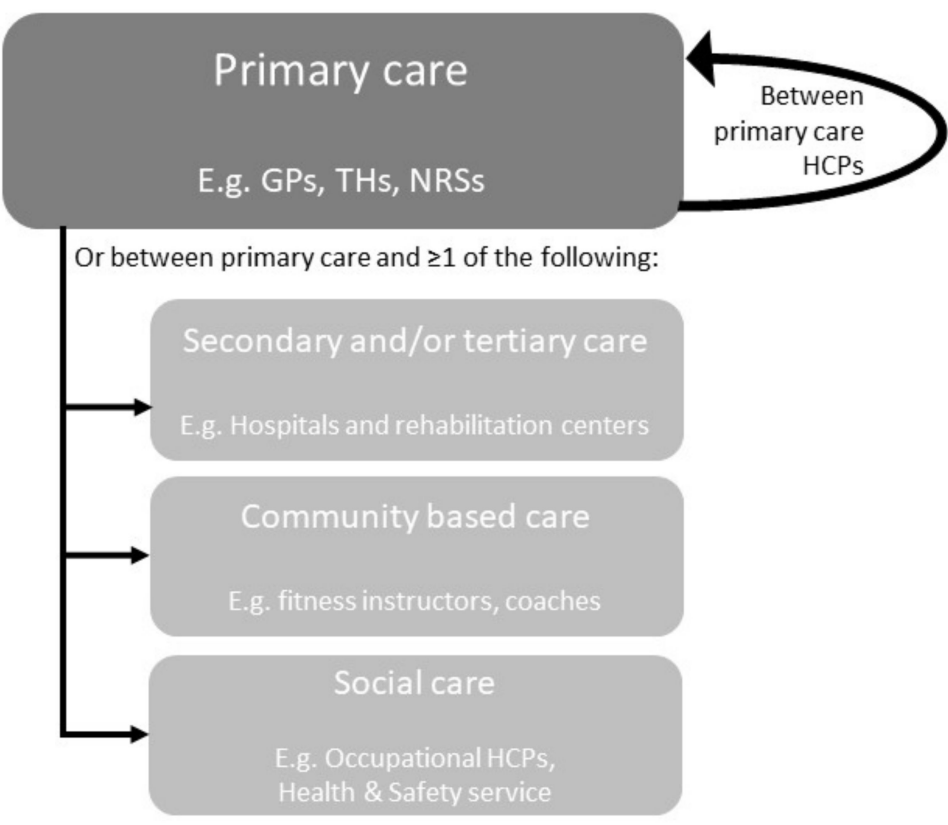

Figure 1. Overview of interdisciplinary care networks. GPs = general practitioners; THs = therapists; NRSs = nurses; $\mathrm{HCPs}=$ health care professionals.

\subsection{Databases Searched and Inclusion and Exclusion Criteria}

Studies published between the 1 January 1994 and the 14 November 2019 were identified by searching the databases PubMed, CINAHL, Web of Science, and PsycInfo, and tracing publications from the reference sections of included papers and relevant reviews. Studies were included if the main population comprised patients with CMP, the intervention was implemented in primary care, or a combination of primary care and other health care settings, with a rehabilitation aim and an interdisciplinary care network. Only original descriptions of interventions in Dutch, English, or German were included. Detailed definitions of the in- and exclusion criteria can be found in Table 1. In addition to these criteria, studies with populations comprising a mix of patients with subacute and chronic complaints or with a non-disease-specific intervention were included. From these studies, only the results for patients with CMP were taken into account. Studies investigating group interventions delivered at the same time by HCPs of different disciplines were also included in this review as it was assumed that they would have discussed treatment approaches. In all interventions, the collaboration between HCPs had to be bidirectional to be included. When other articles described the same intervention, these were also included in our review.

\subsection{Search Strategy}

Terms used were defined by scoping searches and team discussions. An information specialist finalized the search strategy and adapted keywords according to the configuration of each database. Our search strategy has been published online in detail (Appendix A). Briefly, it included variations on the following terms: 'chronic musculoskeletal pain, fibromyalgia, regional pain, arthritis, interdisciplinary, integrated, multidisciplinary, service, system, delivery, network, physical and rehabilitation medicine, Quadruple Aim, health outcome, quality of care, healthcare costs and satisfaction with work'. Three reviewers (CL (100\%), WM (75\%), and LB $(25 \%))$ independently screened title and abstract, and two reviewers (CL $(100 \%)$ and LB $(100 \%))$ screened all full texts. Disagreements were solved by an arbitrator (IH). Iden- 
tified references were downloaded and collected using EndNote bibliographic software (Clarivate Analytics, Philadelphia, PA, USA), and the article selection was performed in the review processing software, Rayyan [29].

Table 1. Inclusion and exclusion criteria.

\begin{tabular}{cc}
\hline \multicolumn{1}{c}{ Inclusion } & Exclusion \\
\hline $\begin{array}{c}\text { An intervention for patients with chronic } \\
\text { musculoskeletal pain (CMP) of the posture- } \\
\text { and locomotion apparatus. Studies were also } \\
\text { included if the study population was a mix of } \\
\text { patients with subacute and chronic complaints. }\end{array}$ & $\begin{array}{c}\text { An intervention developed for headache or } \\
\text { stomach-ache, or only for patients with } \\
\text { subacute pain (<12 weeks). }\end{array}$ \\
\hline $\begin{array}{c}\text { Rehabilitation care enabling individuals aged } \\
\text { 18 years to maintain or return to their daily } \\
\text { life activities, fulfill meaningful life roles and } \\
\text { maximize their well-being [30]. The goal of the } \\
\text { rehabilitation is on the improvement of } \\
\text { participation or functioning of the patient. }\end{array}$ & $\begin{array}{c}\text { A (rehabilitation) intervention which was } \\
\text { designed for pre-post surgery care, or if it } \\
\text { consisted of eHealth, which substitutes the } \\
\text { treatment given by an HCP, or }\end{array}$ \\
$\begin{array}{c}\text { if the intervention only focuses on medication } \\
\text { prescription or use. }\end{array}$
\end{tabular}

An interdisciplinary care network based on the

IASP definition [21]: a multimodal treatment provided by a multidisciplinary team collaborating in assessment and/or treatment using a shared biopsychosocial model and goals. The HCPs all have to work closely together with regular team meetings (face to face or online), agreement on the diagnosis, therapeutic aims and plans for treatment and review.

There was a bidirectional discussion or exchange of treatment approaches with the same goal between HCPs of different

An intervention in which HCPs of different disciplines treated a patient but without a mutual goal, bidirectional discussion, or exchange of treatment approaches.

An intervention that focuses only on the referral or triage of patients without collaboration during the treatment itself. An intervention with only extended practices roles, e.g., the physiotherapist takes over the roles of the GP.

disciplines (e.g., a GP with a physiotherapist).

Implemented within primary care or between

primary care and other healthcare settings

(secondary or tertiary care, social care, or

Interventions implemented within or between secondary or tertiary clinic(s). community-based care) (see Figure 1)

Original descriptions of (results of) an intervention, such as protocol articles, feasibility studies, process evaluations, and qualitative and quantitative (cost)-effectiveness studies.
A review or guideline. The references for these studies were checked for eligible articles.

Only full texts which were available in Dutch, English, or German.

Articles published between 1 January 1994 and 14 November 2019.

\subsection{Data Extraction and Analysis}

CL extracted data on the interdisciplinary care networks, study aims, and outcomes of the included articles. LB reviewed $25 \%$ of the data extraction. First, descriptions of the included interventions were compiled. These included: country; name of intervention; target population; health care setting; and description of the collaboration and intervention. If multiple articles were published for one intervention, these were merged to give a complete overview. As shown in Figure 1, health care settings were classified based on the type of intervention: within primary care or between primary care and secondary or tertiary care, community-based care, and/or social care. Interventions in secondary or tertiary care were combined as one category because the distinction between secondary or tertiary care was not always clear from the descriptions given. Descriptions of interventions were extracted from the studies and classified into these categories: 
- Assessment (a systematic approach to ensuring that the health service uses its resources to improve the health of the population most efficiently) [31];

- Education-basic knowledge (anatomy, biomechanics, the function of the body, and pathophysiology) [32];

- Education-knowledge of disease prevention and ergonomics (information on prevention, cause of pain, ergonomics, information on posture, information on activity, exercise) [32];

- Education-knowledge of treatment (self-management, lifestyle modification, information on coping with the problems) [32];

- Manual Therapy (passive joint mobilization and massage therapy) [33];

- Specific Exercise Therapy (active and/or active-assisted strengthening, mobilizing, and stretching exercises to restore the function of the affected region) [34];

- General Exercise Therapy (aerobic and resistance training, causing an increase in energy expenditure, to maintain health-related outcomes) [35];

- Mind-Body Exercise Therapy (to enhance the mind's capacity to positively affect bodily functions and symptoms, including pain, by combining exercises with mental focus) [36];

- Cognitive behavioral therapy (CBT) (integration of exercise therapy with daily performed activities based on cognitive-behavioral principles, time-contingent) [37-41];

- Workplace intervention (a set of comprehensive health promotion and occupational health strategies implemented in the workplace to improve work-related outcomes) [42];

- Anesthetics (local anesthetics for diagnosis and therapy, indications include functional disorders, inflammatory diseases, and acute and chronic pain) [43];

- Medication management (a systematic process of ensuring that the patient's medication regimen is optimally appropriate, effective, and safe, and that the patient is adhering to this regimen to promote health and reduce the need for health care use) [44].

Second, outcomes relevant to the Quadruple Aim were extracted for each intervention. For these, study dates, study designs, outcome measures with measurement instruments for relevant primary outcomes, and results were recorded. The results of randomized trial designs were presented in two categories: either (1) positive and significant $(+)(p<0.05)$ compared to the comparator intervention for randomized controlled trial (RCT) designs; or (2) positive and non-significant $(p>0.05)$, no difference between the intervention and comparator intervention, or alternatively negative and significant for the intervention compared to the comparator intervention $(-)(p<0.05)$. For non-randomized trial designs, results were classed as significant $(+)(p<0.05)$ or non-significant $(-)(p>0.05)$, compared to baseline. Mixed positive and negative results for subdomains are indicated by $+/-$. For the qualitative studies, opinions are summarized as all positive $(+)$, negative $(-)$, neutral $(=)$, or mixed (+/-). All outcomes from the studies relevant to the Quadruple Aim are presented. Primary outcomes of the studies were identified using the following procedure. First, the primary outcome, as described by the authors, was chosen. If this was not described, the outcome measure used in the sample size calculation was chosen. If this was also not described in the article, the outcome measure best fitting the aim of the intervention was chosen (e.g., aim: improving functioning, outcome measure: health-related quality of life or functioning; aim: return to work, outcome measure: return to work or sick leave; etc.). In the case where this was also unclear from the article, the first choice was the outcome measure for quality of life (often measured in this type of study, making it comparable). Then, costs such as sick leave or return to work were the second choice. After that, quality of care experienced by patients or $\mathrm{HCP}$ work satisfaction were the third choice. Based on the homogeneity and the chosen outcome measures, a meta-analysis was considered.

\subsection{Risk of Bias in Individual Studies}

Quality assessment tools specific to the method(s) employed were used. These tools were used to assess and compare the quality of RCT designs, non-randomized study designs and qualitative designs. For RCT designs, the risk of bias was assessed using Version 2 of the Cochrane risk-of-bias tool for randomized trials (RoB2 tool) [45]. The Risk 
of Bias in Non-randomized Studies-of Interventions (ROBINS-I) tool was used for assessing non-randomized study designs [46]. Domains not relevant for studies without a control group are marked (-) or (NA). For qualitative designs, the Joanna Briggs Institute Critical Appraisal Tools, Checklist for Qualitative Research, was used for a critical appraisal [47]. This critical appraisal tools assist in assessing the trustworthiness, relevance and results of published papers. At least seven questions (out of 10) had to be answered "yes" to receive a positive overall appraisal. Articles describing study protocols were not assessed for risk of bias. One researcher (CL) assessed the risk of bias and performed the critical appraisal for each study. One researcher (LB) randomly cross-checked $25 \%$ of the included studies. Disagreements were resolved by an arbitrator (IH). Review authors were not blinded for author names, institutions, or journals. If additional information was needed, corresponding authors would have been contacted. Results are reported through graphical representation of bias judgements grouped by design of study.

\section{Results}

The process of the literature review is shown in Figure 2. The combination of keywords yielded 15,428 potentially relevant articles in the databases on 14 November 2019. Overall, 2926 articles were excluded by deduplication, and 11 studies were added with the snowball method, resulting in 12,513 articles. After reviewing the titles and abstracts of the articles, 12,152 of these were excluded. In total, 361 full-text articles were assessed for eligibility, and 320 were excluded for not meeting one or more of the criteria. The most common reasons for exclusion were interventions without an interdisciplinary care component or interdisciplinary interventions without a role for primary care. If interventions were described in other articles as protocols or allied studies, these were also included, leading to 49 included articles describing 34 interventions.

\subsection{Overview of Included Studies}

An overview of the included interventions is presented in Table 2. Of the 34 included, 21 consisted of a collaboration of HCPs within primary care [25,48-76]. Examples of these were collaborations between therapists (TH) and nurse practitioners (NP) or more extensive collaborations between physicians / physiatrists (PH), psychologists (PSY), and various THs. These collaborations ranged from merely performing an assessment to giving a complete interdisciplinary treatment in primary care. Nearly all interventions included at least one education module. Only three had a medication management module. Moreover, most studies with collaborating THs had general or specific exercise therapy modules in the intervention. If the interventions had a PSY or Psychosocial counsellor (PSY-C) in the team, these interventions were often focused on Mind-Body Exercise Therapy or CBT. 


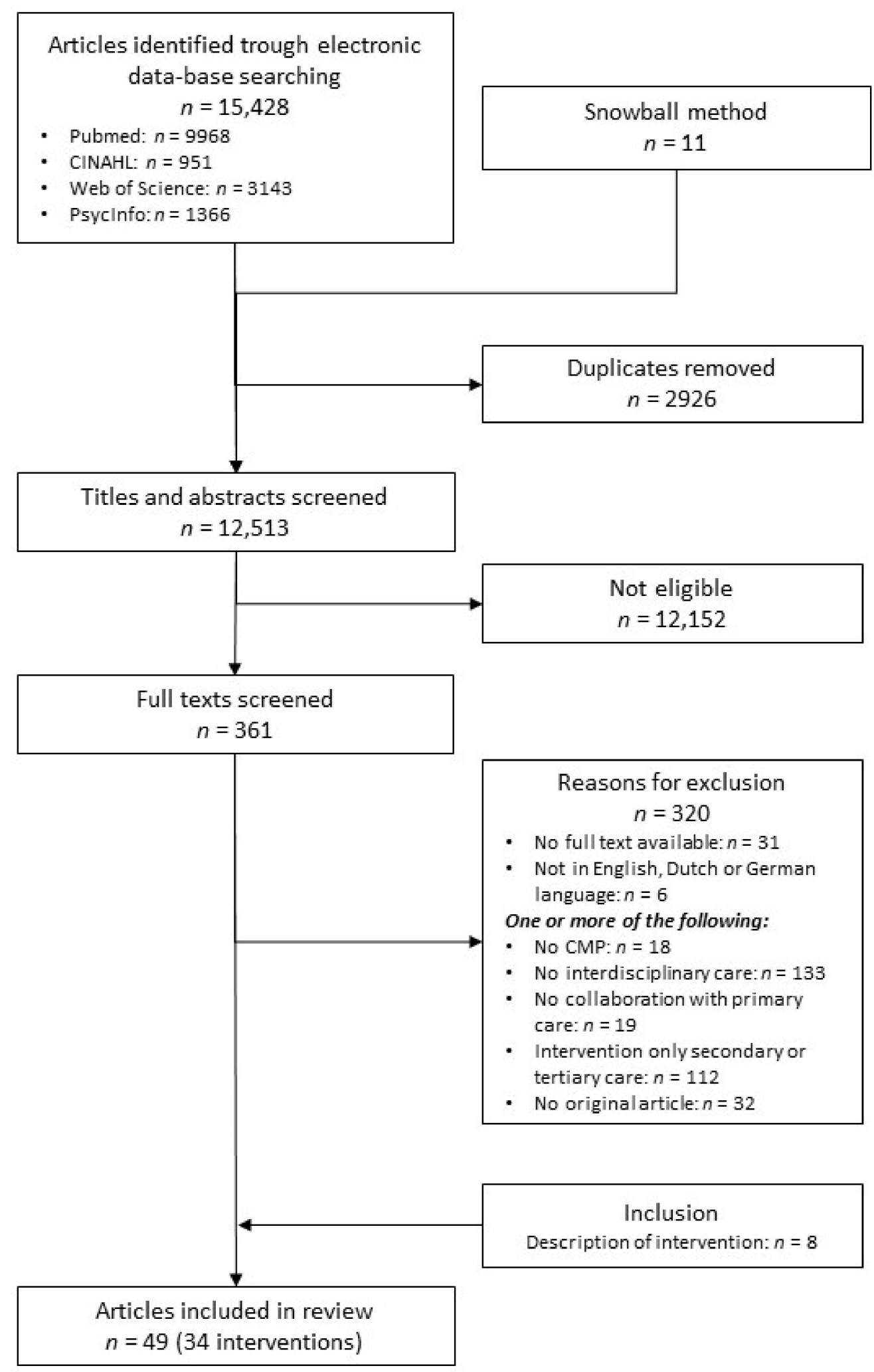

Figure 2. Process of literature selection. 
Table 2. Overview of included studies.

\begin{tabular}{|c|c|c|c|c|c|}
\hline No. & Author, Year \& Country & Intervention Name & Target Population & Collaboration & Content and Intensity Intervention \\
\hline \multicolumn{6}{|c|}{ Within primary care } \\
\hline \multicolumn{6}{|c|}{ Randomized trial designs } \\
\hline 1 & $\begin{array}{l}\text { Calner et al., (2016) [49] } \\
\text { * Intervention is linked to } \\
\text { interventions of Nordin } \\
\text { (number 8) } \\
\text { Sweden }\end{array}$ & $\begin{array}{l}\text { Multimodal pain } \\
\text { Rehabilitation (MMR) \& web } \\
\text { behaviour change program } \\
\text { for activity (Web-BCPA) }\end{array}$ & $\begin{array}{l}\text { Chronic musculoskeletal pain } \\
\text { of the back, neck, shoulders, } \\
\text { and/or a generalized pain } \\
\text { condition }\end{array}$ & $\begin{array}{c}\text { PH } \\
\text { THs } \\
\text { PSY or PSY-C } \\
\text { NRS } \\
1 \times \text { Team discussion with } \\
\text { patient about treatment plan }\end{array}$ & $\begin{array}{c}\text { MMR } \\
\geq 2 \times / \mathrm{w}, \geq 6 \mathrm{w} \\
\text { At least } 3 \text { different healthcare professionals } \\
\text { Specific Exercise Therapy } \\
\text { General Exercise Therapy } \\
\text { Manual Therapy } \\
\text { Mind-Body Exercise Therapy } \\
\text { Web-BCPA } \\
24 \mathrm{~h}, 7 \mathrm{~d}, 16 \mathrm{w} \\
\text { Self-guided by the patient } \\
\text { Education-Knowledge of disease prevention and } \\
\text { ergonomics } \\
\text { Education-Basic knowledge } \\
\text { Education-Knowledge of treatment } \\
\text { Cognitive-behavioral therapy }\end{array}$ \\
\hline 2 & $\begin{array}{l}\text { Chelimsky et al., (2013) [71] } \\
\text { US }\end{array}$ & $\begin{array}{c}\text { Primary Practice Physician } \\
\text { Program for Chronic Pain } \\
\text { (4PCP) }\end{array}$ & $\begin{array}{l}\text { Chronic Pain (back pain } \\
51.9 \% \text {, fibromyalgia } 23.1 \% \text {, } \\
\text { neck pain } 6.7 \% \text {, others) }\end{array}$ & $\begin{array}{l}\text { PH } \\
\text { PSY } \\
\text { THs }\end{array}$ & $\begin{array}{c}\text { * No separate intervention for patients } \\
\text { Collaborative training of PHs consisting of: } \\
\text { Active learning: Evidence-based active learning } \\
\text { seminars, self-directed learning } \\
\text { Clinical support: to collaborate with the } \\
\text { interdisciplinary treatment team comprising } \\
\text { pain-informed THs and PSY providing } \\
\text { cognitive-behavioral therapy }\end{array}$ \\
\hline 3 & $\begin{array}{c}\text { DeBar et al., (2018) [77] } \\
\text { USA }\end{array}$ & $\begin{array}{l}\text { Pain Program for Active } \\
\text { Coping and Training } \\
\text { (PPACT) }\end{array}$ & $\begin{array}{l}\text { Chronic pain } \\
\text { On opioid treatment }(\geq 6 \mathrm{~m}) \\
\text { On health plan }\end{array}$ & $\begin{array}{c}\text { PPACT interventionist team: } \\
\text { PSY-C } \\
\text { NCM } \\
\text { PCPs: } \\
\text { PS } \\
\text { PR } \\
\text { PPACT interventionists and } \\
\text { PCPs meetings for treatment } \\
\text { plan (before start treatment) } \\
\text { and evaluation (end of } \\
\text { treatment) }\end{array}$ & $\begin{array}{c}\text { Comprehensive intake evaluation } \\
\text { NCM or PSY-C } \\
\text { Assessment } \\
\text { Medication management } \\
1 \times \text { TS } \\
\text { Education-Knowledge of disease prevention and } \\
\text { ergonomics } \\
\text { Education-Knowledge of treatment } \\
\text { Cognitive-behavioral therapy (CBT)-based pain } \\
\text { coping skills training and adapted movement } \\
\text { practice } \\
12 \mathrm{w} \text { (group) } \\
\text { Cognitive-behavioral therapy } \\
\text { PCP consultation and patient outreach } \\
\text { By NCM and PSY-C }\end{array}$ \\
\hline
\end{tabular}


Table 2. Cont.

\begin{tabular}{|c|c|c|c|c|c|}
\hline No. & Author, Year \& Country & Intervention Name & Target Population & Collaboration & Content and Intensity Intervention \\
\hline 4 & $\begin{array}{l}\text { 1: Dobscha et al., (2008) [67] } \\
\text { 2: Dobscha et al., (2009) [72] } \\
\text { USA }\end{array}$ & $\begin{array}{c}\text { Study of the Effectiveness of } \\
\text { a Collaborative Approach to } \\
\text { Pain } \\
\text { (SEACAP) }\end{array}$ & $\begin{array}{l}\text { Musculoskeletal pain } \\
\text { Chronic } \\
\text { Exclusion: fibromyalgia }\end{array}$ & $\begin{array}{c}\text { PSY: care manager } \\
\text { IT: intervention \& workshop } \\
\text { teacher } \\
\text { TH: workshop teacher } \\
\text { Discussion between PSY and } \\
\text { IT about assessment results } \\
\text { and treatment } \\
\text { recommendations. These are } \\
\text { sent by email to clinicians. } \\
\text { Leading workshop with PSY } \\
\text { and IT or TH. }\end{array}$ & $\begin{array}{c}\text { Telephone contact } \\
\text { Written materials } \\
\text { Education-Basic knowledge } \\
\text { Assessment } \\
\text { by PSY } \\
\text { Assessment } \\
\text { Education - Knowledge of treatment } \\
\text { Recommendation treatment plan } \\
\text { Based on discussions about symptoms or } \\
\text { additional education by PSY and IT } \\
\text { Workshop } \\
90 \text { m, } 4 \times, 4 \text { months } \\
\text { By PSY, co-led by IT or TH } \\
\text { Education-Knowledge of disease prevention and } \\
\text { ergonomics } \\
\text { Education-Knowledge of treatment }\end{array}$ \\
\hline 5 & $\begin{array}{l}\text { Gustavsson et al., (2018) [69] } \\
\text { Sweden }\end{array}$ & $\begin{array}{c}\text { Activity and life-role } \\
\text { targeting rehabilitation } \\
\text { (ALAR) }\end{array}$ & $\begin{array}{c}\text { Musculoskeletal pain } \\
\text { Chronic }\end{array}$ & $\begin{array}{c}\text { TSs } \\
\text { PH } \\
\text { PSY-C } \\
\text { TSs: Participating in } \\
\text { education meetings about } \\
\text { treatment protocol and } \\
\text { behavioral medicine } \\
\text { approach, } 3 \times, 4 \mathrm{~h} \\
\text { MMR: team discussions } \\
\text { about assessment and } \\
\text { treatment plan }\end{array}$ & $\begin{array}{c}\text { Multimodal pain rehabilitation (MMR) } \\
\text { Content and intensity are patient dependent } \\
\text { Assessment } \\
\text { Cognitive-behavioral therapy } \\
\text { ALAR + MMR } \\
1 \mathrm{~h}, 10 \times, 10 \mathrm{w} \\
\text { Workbook and therapist for goal setting } \\
\text { Assessment } \\
\text { Education-Knowledge of treatment } \\
\text { Cognitive-behavioral therapy }\end{array}$ \\
\hline 6 & $\begin{array}{c}\text { Hansson et al., (2010) [75] } \\
\text { Sweden }\end{array}$ & $\begin{array}{l}\text { Patient education program } \\
\text { for osteoarthritis (PEPOA) }\end{array}$ & OA in hip, knee or hand & $\begin{array}{c}\text { THs } \\
\text { OS } \\
\text { NRS } \\
\text { DT } \\
\text { Providing PEPOA }\end{array}$ & $\begin{array}{c}\text { PEPOA }(n=8-10) \\
3 \mathrm{~h}, 5 \times, 1 \times / \mathrm{w}, 5 \mathrm{w} \\
\text { Education-Knowledge of treatment }\end{array}$ \\
\hline
\end{tabular}


Table 2. Cont

\begin{tabular}{|c|c|c|c|c|c|}
\hline No. & Author, Year \& Country & Intervention Name & Target Population & Collaboration & Content and Intensity Intervention \\
\hline 7 & $\begin{array}{l}\text { 1: Helminen et al., (2013) [78] } \\
\text { 2: Helminen et al., (2015) [73] } \\
\text { Finland }\end{array}$ & $\begin{array}{c}\text { Cognitive-behavioural }(\mathrm{CB}) \\
\text { intervention for } \mathrm{OA}\end{array}$ & $\begin{array}{l}\text { Knee pain } \\
\text { Chronic }\end{array}$ & $\begin{array}{c}\text { PSY } \\
\text { TH } \\
\text { Providing CB intervention }\end{array}$ & $\begin{array}{l}\text { Cognitive Behavioral group intervention } \\
\qquad(n=8-10): \\
1 \times / \mathrm{w}, 2 \mathrm{~h}, 6 \mathrm{w} \\
\text { Education-Basic knowledge } \\
\text { Education-Knowledge of disease prevention and } \\
\text { ergonomics } \\
\text { Education-Knowledge of treatment } \\
\text { Mind-Body Exercise Therapy }\end{array}$ \\
\hline 8 & $\begin{array}{l}\text { 1: Nordin et al., (2016) [74] } \\
\text { 2:Nordin et al., (2017) [79] } \\
\text { Sweden }\end{array}$ & $\begin{array}{l}\text { Web Behavior Change } \\
\text { Program for Activity } \\
\text { (Web-BCPA) added to } \\
\text { multimodal pain } \\
\text { rehabilitation (MMR) }\end{array}$ & $\begin{array}{c}\text { Pain in the back, neck, } \\
\text { shoulder, and/or generalized } \\
\text { pain }\end{array}$ & $\begin{array}{c}\text { NRS } \\
\text { THs } \\
\text { PH } \\
\text { PSY } \\
\text { PSY-C } \\
\text { PH for contact with the team } \\
\text { and Swedish Social Insurance } \\
\text { Agency }\end{array}$ & $\begin{array}{c}\text { MMR } \\
2-3 \times / \mathrm{w}, 6-8 \mathrm{w} \\
\text { By } \geq 3 \text { disciplines } \\
\text { Specific Exercise Therapy } \\
\text { General Exercise Therapy } \\
\text { Manual Therapy } \\
\text { Education—Knowledge of disease prevention and } \\
\text { ergonomics } \\
\text { Education-Knowledge of treatment } \\
\text { Cognitive-behavioral therapy } \\
\text { Mind-Body Exercise Therapy } \\
\text { Web-BCPA } \\
16 \mathrm{w} \\
\text { Self-guided } \\
\text { Cognitive-behavioral therapy } \\
\text { Education-Knowledge of treatment }\end{array}$ \\
\hline
\end{tabular}


Table 2. Cont.

\begin{tabular}{|c|c|c|c|c|c|}
\hline No. & Author, Year \& Country & Intervention Name & Target Population & Collaboration & Content and Intensity Intervention \\
\hline \multicolumn{6}{|c|}{ Non-randomized trial designs } \\
\hline 9 & $\begin{array}{l}\text { 1: Dunstan et al., (2007) [80] } \\
\text { 2: Dunstan et al., (2014) [81] } \\
\text { Australia }\end{array}$ & $\begin{array}{l}\text { Light multidisciplinary } \\
\text { Work-Related Activity } \\
\text { Program (WRAP) }\end{array}$ & $\begin{array}{l}\text { Musculoskeletal pain } \\
\text { Chronic }\end{array}$ & $\begin{array}{l}\text { PSY } \\
\text { TH } \\
\text { GP } \\
\text { ORP }\end{array}$ & $\begin{array}{c}\text { WRAP }(n=30,7 \text { groups }) \\
4 \mathrm{~h}, 1 \times / \mathrm{w}, 6 \mathrm{w} \\
\text { By PSY and TH providing treatment, GP as } \\
\text { medical case-manager, and ORP as a } \\
\text { return-to-work case manager } \\
\text { Mind-Body Exercise Therapy } \\
\text { Specific Exercise Therapy } \\
\text { General Exercise Therapy } \\
\text { Education-Basic knowledge } \\
\text { Education-Knowledge of disease prevention and } \\
\text { ergonomics } \\
\text { Workplace intervention }\end{array}$ \\
\hline 10 & $\begin{array}{c}\text { Gurden et al., (2012) [82] } \\
\text { UK }\end{array}$ & $\begin{array}{l}\text { North East Essex Primary } \\
\text { Care Trust manual therapy } \\
\text { service }\end{array}$ & $\begin{array}{l}\text { Back or Neck pain } \\
\text { Subacute and chronic }\end{array}$ & $\begin{array}{c}\text { GP } \\
\text { CH } \\
\text { THs } \\
\text { Prescribed treatment plan } \\
\text { Advise during referral after } \\
\text { treatment (TH to GP) }\end{array}$ & $\begin{array}{c}\text { GP consultation } \\
\text { Assessment } \\
\text { Education-Basic knowledge } \\
\text { Medication management } \\
\text { Manual therapy (within 2 weeks) } \\
\text { max. 6× } \\
\text { CH, TSs } \\
\text { Manual Therapy } \\
\text { Discharge with a report to GP }\end{array}$ \\
\hline 11 & $\begin{array}{l}\text { 1: Mårtensson et al., } \\
\quad \text { (1999) [83] } \\
\text { 2: Mårtensson et al., } \\
\text { (2004) [84] } \\
\text { 3: Mårtensson et al., } \\
\quad \text { (2006) [85] } \\
\quad \text { Sweden }\end{array}$ & $\begin{array}{l}\text { Biopsychosocial } \\
\text { rehabilitation programme, } \\
\text { Focus on Health }(\mathrm{FoH})\end{array}$ & $\begin{array}{l}\text { Pain } \\
\text { Chronic }\end{array}$ & $\begin{array}{c}\text { GP } \\
\text { NRS } \\
\text { THs } \\
\text { PSY-C } \\
\text { Teaching in FoH program }\end{array}$ & $\begin{array}{c}\text { FoH Group sessions }(n=5-9) \\
2 \times / \mathrm{w}, 6 \mathrm{w}: 6 \times 6 \mathrm{~h}+6 \times 3 \mathrm{~h} \\
\text { Education-Basic knowledge } \\
\text { Education-Knowledge of disease prevention and } \\
\text { ergonomics } \\
\text { Mind-Body Exercise Therapy } \\
\text { Education-Knowledge of treatment } \\
\text { Ergonomics } \\
\text { Individual introductory and concluding } \\
\text { conversation for activity and locomotion analysis }\end{array}$ \\
\hline
\end{tabular}


Table 2. Cont.

\begin{tabular}{|c|c|c|c|c|c|}
\hline No. & Author, Year \& Country & Intervention Name & Target Population & Collaboration & Content and Intensity Intervention \\
\hline 12 & $\begin{array}{c}\text { Schütze et al., (2014) [86] } \\
\text { Australia }\end{array}$ & $\begin{array}{c}\text { Mindfulness-Based } \\
\text { Functional Therapy (MBFT) }\end{array}$ & $\begin{array}{c}\text { LBP } \\
\text { Chronic }\end{array}$ & $\begin{array}{c}\text { PSY } \\
\text { TH } \\
\text { Co-facilitated sessions }\end{array}$ & $\begin{array}{c}\text { MBFT-group session }(n=6 \& n=10) \\
2 \mathrm{~h} / \mathrm{w}, 8 \mathrm{w} \\
\text { Education-Knowledge of treatment } \\
\text { Mind-Body Exercise Therapy } \\
\text { Cognitive-behavioral therapy }\end{array}$ \\
\hline 13 & $\begin{array}{c}\text { Stein et al., (2013) [87] } \\
\text { Sweden }\end{array}$ & $\begin{array}{l}\text { Multidisciplinary pain } \\
\text { rehabilitation (MDR) }\end{array}$ & $\begin{array}{l}\text { Musculoskeletal pain } \\
\text { Chronic }\end{array}$ & $\begin{array}{c}\text { GP } \\
\text { PSY } \\
\text { THs } \\
\text { Examination report of GP } \\
\text { visible by all } \\
\text { Team meeting about } \\
\text { biopsychosocial motivation } \\
\text { to participate } \\
\text { Providing treatment }\end{array}$ & $\begin{array}{c}\text { MDR Group sessions }(n=6-8) \text { : } \\
5 \mathrm{~h}, 3 \mathrm{~d} / \mathrm{w}, 6 \mathrm{w} \\
\text { GP }(12 \mathrm{~h}) \\
\text { Education-Basic knowledge } \\
\text { Mind-Body Exercise Therapy } \\
\text { TH }(18 \mathrm{~h}) \\
\text { Education—Knowledge of disease prevention and } \\
\text { ergonomics } \\
\text { Mind-Body Exercise Therapy } \\
\text { TH }(20 \mathrm{~h}) \\
\text { Specific Exercise Therapy } \\
\text { Cognitive-behavioral therapy } \\
\text { Mind-Body Exercise Therapy } \\
\text { PSY (28 h) } \\
\text { Education-Knowledge of disease prevention and } \\
\text { ergonomics } \\
\text { Education-Knowledge of treatment } \\
\text { Cognitive-behavioral therapy } \\
\text { Additional education (12 h), provided by Swedish } \\
\text { Insurance Agency, Swedish Employment Agency, } \\
\text { local fitness center, dietary adviser }\end{array}$ \\
\hline
\end{tabular}


Table 2. Cont.

\begin{tabular}{|c|c|c|c|c|c|}
\hline No. & Author, Year \& Country & Intervention Name & Target Population & Collaboration & Content and Intensity Intervention \\
\hline 14 & $\begin{array}{c}\text { Tyack et al., (2013) [88] } \\
\text { Australia }\end{array}$ & $\begin{array}{l}\text { Student-led interdisciplinary } \\
\text { chronic disease health service }\end{array}$ & $\begin{array}{l}\text { Back pain } \\
\text { Chronic }\end{array}$ & $\begin{array}{c}\text { NRS } \\
\text { PO } \\
\text { THs } \\
\text { Exercise PSY } \\
\text { PSY-C } \\
\text { SP } \\
\text { DT } \\
\text { PR } \\
\text { Indigenous health worker } \\
\text { Case conference and service } \\
\text { delivery }\end{array}$ & $\begin{array}{c}\text { Intake } \\
\text { by } 1 \mathrm{HCP} \text { and } 2 \text { students } \\
\text { Assessment } \\
\text { Case conference } \\
\text { By the team } \\
\text { Selection of appropriate services } \\
\text { Services } \\
\text { from one or more HCP } \\
3-6 \text { months }\end{array}$ \\
\hline 15 & $\begin{array}{l}\text { Westman et al., (2006) [89] } \\
\text { Sweden }\end{array}$ & $\begin{array}{l}\text { STAR project; multimodal } \\
\text { rehabilitation program }\end{array}$ & $\begin{array}{c}\text { Musculoskeletal pain } \\
\text { Chronic } \\
\text { Sick listed }\end{array}$ & $\begin{array}{c}\text { PSY } \\
\text { PH } \\
\text { TH } \\
\text { A representative from the } \\
\text { National Insurance Company } \\
\text { Team discussions about } \\
\text { treatment plan }\end{array}$ & $\begin{array}{c}\text { STAR project group based }(n=8-10) \\
3.5 \mathrm{~h} / \mathrm{d}, 5 \mathrm{~d}, 8 \mathrm{w} \\
\text { General Exercise Therapy } \\
\text { Mind-Body Exercise Therapy } \\
\text { Creative activities } \\
\text { Education-Basic knowledge } \\
\text { Education-Knowledge of disease prevention and } \\
\text { ergonomics } \\
\text { Education-Knowledge of treatment } \\
\text { Individual (when necessary): } \\
\text { Physiotherapy or psychotherapy or orthopedic } \\
\text { consultation }\end{array}$ \\
\hline
\end{tabular}


Table 2. Cont.

\begin{tabular}{|c|c|c|c|c|c|}
\hline No. & Author, Year \& Country & Intervention Name & Target Population & Collaboration & Content and Intensity Intervention \\
\hline 16 & $\begin{array}{c}\text { Westman et al., (2010) [90] } \\
\text { Sweden }\end{array}$ & $\begin{array}{l}\text { Multidisciplinary } \\
\text { rehabilitation program }\end{array}$ & $\begin{array}{c}\text { Musculoskeletal pain } \\
\text { Chronic } \\
\text { Sick listed }\end{array}$ & $\begin{array}{c}\text { GP } \\
\text { TH } \\
\text { PSY or PSY-C } \\
\text { Team discussions about } \\
\text { diagnosis and treatment plan }\end{array}$ & $\begin{array}{c}\text { Assessment and deciding treatment program } \\
1 \times / \mathrm{w} \\
\text { By the team } \\
\text { Assessment } \\
\text { And one or more of the following interventions: } \\
\text { Multimodal Group }(n=6-8) \\
4 \mathrm{~h} / \mathrm{d}, 4 \mathrm{~d}, 6 \mathrm{w} \\
\text { General Exercise Therapy } \\
\text { Mind-Body Exercise Therapy } \\
\text { Creative activities } \\
\text { Education-Basic knowledge } \\
\text { Education-Knowledge of disease prevention and } \\
\text { ergonomics } \\
\text { Education-Knowledge of treatment } \\
\text { Three-way communication } \\
\text { Patient, GP/PSY or PSY-C } \\
\text { Adjustments of treatment plan } \\
\text { Individual } \\
\text { TH or PSY or orthopedic consultation } \\
\text { Workplace-based intervention } \\
\text { Workplace intervention }\end{array}$ \\
\hline
\end{tabular}


Table 2. Cont.

\begin{tabular}{|c|c|c|c|c|c|}
\hline No. & Author, Year \& Country & Intervention Name & Target Population & Collaboration & Content and Intensity Intervention \\
\hline \multicolumn{6}{|c|}{ Qualitative designs } \\
\hline 17 & $\begin{array}{c}\text { 1:Dorflinger et al., (2014) [91] } \\
\text { 2: Purcell et al., (2018) [25] } \\
\text { USA }\end{array}$ & Integrated Pain Team (ITP) & $\begin{array}{l}\text { Pain } \\
\text { Chronic }\end{array}$ & $\begin{array}{c}\text { PH and/or NP } \\
\text { PSY } \\
\text { PR } \\
\text { Team discussions about } \\
\text { diagnosis and treatment plan } \\
\text { Providing treatment } \\
\text { Keeping track of treatments } \\
\text { (inside and outside ITP) }\end{array}$ & $\begin{array}{c}\text { ITP existing of: } \\
3 \times, 2-3 \mathrm{~m}, n=15-20 / \mathrm{m} \\
\text { Interdisciplinary Assessment } \\
1 \text { h by complete team and patient } \\
\text { Assessment } \\
\text { Education-Basic knowledge } \\
\text { Medication management } \\
\text { During complete follow-up by ITP } \\
\text { Medication management } \\
\text { Additional } \\
\text { Education-Knowledge of disease prevention and } \\
\text { ergonomics } \\
\text { Education-knowledge of treatment } \\
\text { Cognitive-behavioral therapy }\end{array}$ \\
\hline 18 & $\begin{array}{l}\text { 1: Bath et al., (2016) [65] } \\
\text { 2: Lovo et al., (2019) [92] } \\
\text { Canada }\end{array}$ & $\begin{array}{c}\text { Secure video } \\
\text { conferencing/telehealth }\end{array}$ & $\begin{array}{c}\text { LBP } \\
\text { Chronic }\end{array}$ & $\begin{array}{c}\text { TH (urban-based) } \\
\text { NP (local rural) } \\
1 \times \text { Digital assessment }\end{array}$ & $\begin{array}{c}\text { Digital assessment } \\
1 \times \\
\text { NP at patient side performing a physical } \\
\text { examination } \\
\text { Assessment } \\
\text { Education-Knowledge of treatment }\end{array}$ \\
\hline 19 & $\begin{array}{c}\text { Pietilä Holmner et al., (2018) } \\
\text { [93] } \\
\text { Sweden }\end{array}$ & $\begin{array}{l}\text { Multimodal rehabilitation } \\
\text { (MMR) }\end{array}$ & $\begin{array}{c}\text { Pain } \\
\text { Chronic } \\
\text { Sick listed (or at risk) }\end{array}$ & $\begin{array}{c}\text { THs } \\
\text { PH } \\
\text { PSY } \\
\text { Team discussions about } \\
\text { assessment and treatment }\end{array}$ & $\begin{array}{c}\text { MMR } \\
\text { Individual and/or group intervention } \\
\text { General Exercise Therapy } \\
\text { Mind-Body Exercise Therapy } \\
\text { Education-Knowledge of treatment }\end{array}$ \\
\hline 20 & $\begin{array}{c}\text { Stenberg et al., (2016) [94] } \\
\text { Sweden }\end{array}$ & $\begin{array}{l}\text { Multimodal rehabilitation } \\
\text { (MMR) }\end{array}$ & $\begin{array}{c}\text { Pain } \\
\text { Chronic } \\
\text { Sick listed (or at risk) }\end{array}$ & $\begin{array}{c}\text { THs } \\
\text { PSY-C } \\
\text { PSY } \\
\text { GP } \\
\text { DT } \\
\text { NRS } \\
\text { THs deliver treatment. Other } \\
\text { members deliver treatment or } \\
\text { have a consultation function }\end{array}$ & $\begin{array}{l}\text { MMR } \\
\text { By THs and optionally } \geq 1 \text { of the other HCPs } \\
\text { Group, individually, or combination } \\
\text { Cognitive-behavioral therapy }\end{array}$ \\
\hline
\end{tabular}


Table 2. Cont.

\begin{tabular}{|c|c|c|c|c|c|}
\hline No. & Author, Year \& Country & Intervention Name & Target Population & Collaboration & Content and Intensity Intervention \\
\hline 21 & $\begin{array}{l}\text { 1: Sundberg et al., (2007) [76] } \\
\text { 2: Sundberg et al., (2009) [95] } \\
\text { Sweden }\end{array}$ & $\begin{array}{l}\text { Integrative medicine (IM) } \\
\text { management }\end{array}$ & $\begin{array}{c}\text { Back or Neck pain } \\
\text { Mixed population subacute } \\
\text { and chronic }\end{array}$ & $\begin{array}{c}\text { GP } \\
\text { Senior CT providers } \\
\text { Team discussions about } \\
\text { treatment plan }\end{array}$ & $\begin{array}{c}\text { IM } \\
\text { Conventional therapies, advise by GP } \\
\text { Education-Knowledge of disease prevention and } \\
\text { ergonomics } \\
\text { Anaesthetics } \\
\text { General Exercise Therapy } \\
\text { Complementary therapies by CT providers } \\
\text { Manual Therapy }\end{array}$ \\
\hline \multicolumn{6}{|c|}{ Between primary care and secondary or tertiary care } \\
\hline \multicolumn{6}{|c|}{ Randomized trial designs } \\
\hline 22 & $\begin{array}{c}\text { Haldorsen et al., (1998) [50] } \\
\text { Norway }\end{array}$ & $\begin{array}{l}\text { Multimodal Cognitive } \\
\text { Behavioral Treatment } \\
\text { (MMCBT) }\end{array}$ & $\begin{array}{l}\text { Back, neck, shoulder pain, } \\
\text { generalized muscle pain, } \\
\text { more localized } \\
\text { musculoskeletal disorders } \\
\text { Subacute and chronic } \\
\text { Sick listed }\end{array}$ & $\begin{array}{c}\text { NEU } \\
\text { GP } \\
\text { PSY } \\
\text { Registered NRS } \\
\text { TH } \\
\text { Team discussions on } \\
\text { diagnosis and treatment plan } \\
\text { Providing treatment } \\
\text { (e.g., Education) }\end{array}$ & $\begin{array}{c}\text { Multidisciplinary rehabilitation } \\
6 \mathrm{~h}, 5 \times / \mathrm{w}, 4 \mathrm{w} \\
\text { Combination of group and individual treatment } \\
\text { Assessment } \\
\text { Specific Exercise Therapy } \\
\text { General Exercise Therapy } \\
\text { Individual by TH } \\
\text { Cognitive-behavioral therapy }(8 \times) \\
\text { Education-Basic knowledge } \\
\text { Education-Knowledge of disease prevention and } \\
\text { ergonomics } \\
\text { Education-Knowledge of treatment } \\
2 \times \text { lectures and discussions by all healthcare } \\
\text { professionals } \\
\text { Workplace interventions } \\
\text { By physician, human resource officer, } \\
\text { occupational counsellor, representative of a } \\
\text { governmental social insurance authority. }\end{array}$ \\
\hline
\end{tabular}


Table 2. Cont.

\begin{tabular}{|c|c|c|c|c|c|}
\hline No. & Author, Year \& Country & Intervention Name & Target Population & Collaboration & Content and Intensity Intervention \\
\hline 23 & $\begin{array}{l}\text { Rothman et al., (2013) [52] } \\
\text { Sweden }\end{array}$ & $\begin{array}{l}\text { Multidisciplinary, } \\
\text { multimodal (MM), } \\
\text { multi-professional } \\
\text { assessment }\end{array}$ & $\begin{array}{l}\text { CMP } \\
\text { Chronic }\end{array}$ & $\begin{array}{c}\text { GP } \\
\text { And } \geq 3 \text { : } \\
\text { NRS } \\
\text { PSY } \\
\text { TH } \\
\text { PSY-C } \\
\text { OS } \\
\text { when necessary: } \\
\text { liaison PH at the } \\
\text { Psychosomatic Medicine } \\
\text { Clinic (PMC) } \\
\text { Interdisciplinary team } \\
\text { meeting about assessment }\end{array}$ & $\begin{array}{l}\text { Assessment in the MM Group } \\
\text { Each discipline had } 1 \text { meeting with patient (mean } \\
7 \text { sessions) } \\
\text { Conference meeting to give treatment advice: } \\
\text { - Multidisciplinary group pain management at } \\
\text { the PMC, } \\
\text { Multidisciplinary individual pain manage- } \\
\text { ment at the PMC, } \\
\text { - Multidisciplinary individual pain manage- } \\
\text { ment at GP and associated team or at a multi- } \\
\text { disciplinary clinic. } \\
\text { Assessment }\end{array}$ \\
\hline 24 & $\begin{array}{c}\text { Taylor-Gjevre et al., (2017) } \\
\text { [53] } \\
\text { Canada }\end{array}$ & Video-conferencing & RA & $\begin{array}{c}\text { Urban-based RT } \\
\text { On-site TH } \\
\text { Performing assessment and } \\
\text { follow-up care }\end{array}$ & $\begin{array}{l}\text { Video-conferencing treatment } \\
4 \times \\
\text { TH is at patient side for physical examination and } \\
\text { set up conferencing with the rheumatologist who } \\
\text { is performing the assessment and follow-up care } \\
\text { Assessment }\end{array}$ \\
\hline
\end{tabular}


Table 2. Cont.

\begin{tabular}{|c|c|c|c|c|c|}
\hline No. & Author, Year \& Country & Intervention Name & Target Population & Collaboration & Content and Intensity Intervention \\
\hline \multicolumn{6}{|c|}{ Non-randomized trial designs } \\
\hline 25 & $\begin{array}{c}\text { Burnham et al., (2010) [48] } \\
\text { Canada }\end{array}$ & $\begin{array}{l}\text { Central Alberta Pain and } \\
\text { Rehabilitation Institute } \\
\text { (CAPRI) program }\end{array}$ & $\begin{array}{l}\text { Pain } \\
\text { Chronic }\end{array}$ & $\begin{array}{c}\text { PH } \\
\text { TH } \\
\text { GP } \\
\text { PSY } \\
\text { NRS } \\
\text { DT } \\
\text { KN } \\
\text { Team discussions about } \\
\text { treatment plan } \\
\text { Executing of treatment (full } \\
\text { multidisciplinary } \\
\text { management) }\end{array}$ & $\begin{array}{c}\text { Referral documentation review } \\
\text { GP } \\
\text { Initial assessment } \\
\text { 1: spine care assessment: } 1,5 \mathrm{~h}, \text { by } \mathrm{PH} \text { and } \mathrm{TH} \\
\text { 2: medical care assessment (optional): } 2 \mathrm{~h} \text {, by GP } \\
\text { Assessment } \\
\text { Treatment (1 of the options) } \\
\text { I-1: Consultation only: education, activity } \\
\text { modification, and a customized home exercise } \\
\text { program } \\
\text { Education } \\
\text { General exercise therapy } \\
\text { by PH } \\
\text { Anaesthetics } \\
\text { I-3: Supervised medication management: by GP } \\
\text { Medication management } \\
\text { I-4: Full multidisciplinary management }(n=4-6) \text { : } \\
5 \mathrm{~h}, 1 \times / \mathrm{w}, 2-3 \text { months, the whole team } \\
\text { Group discussions about education and treatment } \\
\text { plan } \\
\text { Psychotherapy } \\
\text { Education-Basic knowledge } \\
\text { Education-Knowledge of treatment }\end{array}$ \\
\hline 26 & $\begin{array}{c}\text { Claassen et al., (2018) [49] } \\
\text { The Netherlands }\end{array}$ & Osteoarthritis (OA) education & OA in hip or knee & $\begin{array}{c}\text { GP } \\
\text { TH } \\
\text { OS or NP } \\
\text { Public health advisor (when } \\
\text { available) } \\
\text { Teaching in OA educational } \\
\text { program }\end{array}$ & $\begin{array}{c}\text { OA educational program }(n=10-12) \\
1,5 \mathrm{~h}, 2 \times \\
\text { Education-Knowledge of disease prevention and } \\
\text { ergonomics } \\
\text { Education-Basic knowledge } \\
\text { Education - Knowledge of treatment } \\
\text { Booklet } \\
\text { Information, monitoring forms, course handout, } \\
20 \text { FAQs, a pedometer, and a list of websites and } \\
\text { contact information }\end{array}$ \\
\hline
\end{tabular}


Table 2. Cont.

\begin{tabular}{|c|c|c|c|c|c|}
\hline No. & Author, Year \& Country & Intervention Name & Target Population & Collaboration & Content and Intensity Intervention \\
\hline 27 & $\begin{array}{c}\text { Plagge et al., (2013) [51] } \\
\text { USA }\end{array}$ & $\begin{array}{l}\text { Integrated Management of } \\
\text { Pain and PTSD in Returning } \\
\text { OEF/OIF/ONDVEterans } \\
\text { (IMPPROVE) }\end{array}$ & $\begin{array}{c}\text { Pain } \\
\text { Chronic } \\
\text { Posttraumatic stress disorder } \\
\text { Veterans }\end{array}$ & $\begin{array}{c}\text { PSY } \\
\text { PH } \\
\text { Discussions about assessment } \\
\text { and weekly telephone } \\
\text { meetings about treatment }\end{array}$ & $\begin{array}{c}\text { Biopsychosocial evaluation } \\
90 \text { min by PSY } \\
\text { Assessment } \\
\text { Care management } \\
1 \times / \mathrm{w} \text { by PSY and PH } \\
\text { Reviewing recommendations with veterans, } \\
\text { assessing interest and willingness to engage in } \\
\text { recommended treatments, discussing concerns or } \\
\text { questions, coordination of care between services, } \\
\text { facilitating communication between the veteran } \\
\text { and providers, helping veterans navigate the VA } \\
\text { system, monitoring treatment plans } \\
\text { Behavioral Activation Psychotherapy } \\
8 \times, 75-90 \text { min } \\
\text { Individual by PSY } \\
\text { Cognitive-behavioral therapy }\end{array}$ \\
\hline
\end{tabular}


Table 2. Cont

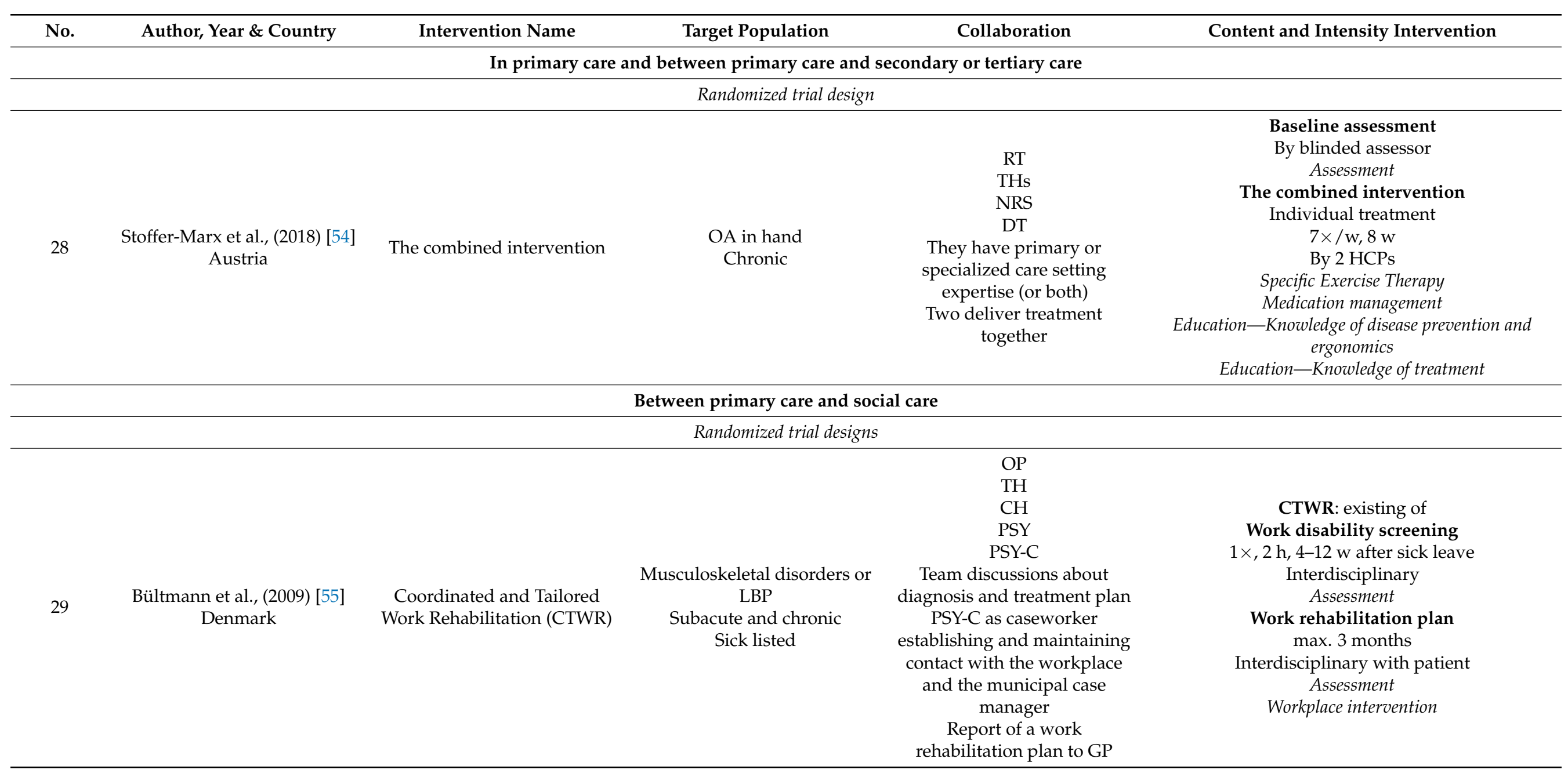


Table 2. Cont.

\begin{tabular}{|c|c|c|c|c|c|}
\hline No. & Author, Year \& Country & Intervention Name & Target Population & Collaboration & Content and Intensity Intervention \\
\hline \multicolumn{6}{|c|}{ Non-randomized trial design } \\
\hline 30 & $\begin{array}{l}\text { Heijbel et al., (2013) [56] } \\
\text { Sweden }\end{array}$ & $\begin{array}{l}\text { Occupational Health Service } \\
\text { (OHS) }\end{array}$ & $\begin{array}{c}\text { Mixed group } \\
\text { Musculoskeletal problems } \\
\text { Subacute and chronic } \\
\text { Sick listed }\end{array}$ & $\begin{array}{c}\text { PH } \\
\text { TH } \\
\text { PSY } \\
\text { NRS } \\
\text { Team assessment }\end{array}$ & $\begin{array}{c}\text { Team assessment } \\
\text { With team } \\
\text { Assessment } \\
\text { Rehabilitation meeting and plan of measures } \\
\text { With patient, supervisor, several OHS team } \\
\text { members, local insurance office, trade union } \\
\text { (optional) } \\
\text { Treatment } \\
\text { Education } \\
\text { Cognitive-behavioral therapy } \\
4 \mathrm{w}, \text { FU } 6 \mathrm{~m} \text { or } 12 \mathrm{~m} \\
\text { Workplace intervention } \\
\text { Follow-up meeting after rehabilitation }\end{array}$ \\
\hline \multicolumn{6}{|c|}{ Between primary care and secondary or tertiary care and social care } \\
\hline \multicolumn{6}{|c|}{ Randomized trial designs } \\
\hline
\end{tabular}


Table 2. Cont.

\begin{tabular}{|c|c|c|c|c|c|}
\hline No. & Author, Year \& Country & Intervention Name & Target Population & Collaboration & Content and Intensity Intervention \\
\hline 32 & $\begin{array}{l}\text { 1: Steenstra et al., (2003) [61] } \\
\text { 2: Anema et al., (2007) [57] } \\
\text { *Interventions are identical to } \\
\text { the interventions of Lambeek. } \\
\quad \text { (number 31) } \\
\text { The Netherlands }\end{array}$ & $\begin{array}{l}\text { Workplace intervention and } \\
\text { Graded Activity }\end{array}$ & $\begin{array}{c}\text { Non-specific LBP } \\
\text { Subacute and chronic } \\
\text { Sick listed }\end{array}$ & $\begin{array}{c}1: \\
\text { OP } \\
\text { GP } \\
\text { Contact about referral } \\
\text { OP } \\
\text { GP } \\
\text { THs } \\
\text { Workplace intervention with } \\
\text { worker, employer, OP, GP }\end{array}$ & $\begin{array}{c}\text { Combined intervention (CI): existing of } \\
\text { Workplace intervention (WI) } \\
\text { direct after inclusion (2-6 weeks after sick leave) } \\
24 \mathrm{~d} \\
\text { Assessment } \\
\text { Workplace intervention } \\
\text { Graded Activity Program (optional) } \\
0.5 \mathrm{~h}, 2 \times / \mathrm{w} \text {, max. } 26 \times \\
\text { After } 8 \text { weeks of sick leave } \\
\text { By TH } \\
\text { Cognitive-behavioral therapy }\end{array}$ \\
\hline \multicolumn{6}{|c|}{ Between primary care and community-based care } \\
\hline \multicolumn{6}{|c|}{ Randomized trial designs and qualitative designs } \\
\hline 33 & $\begin{array}{l}\text { 1: McBeth et al., (2012) [66] } \\
\text { 2: Bee et al., (2016) [62] } \\
\text { England }\end{array}$ & $\begin{array}{l}\text { Combined Cognitive } \\
\text { Behavioral Therapy (T-CBT) } \\
\text { and prescribed exercise (PE) }\end{array}$ & $\begin{array}{l}\text { Fibromyalgia } \\
\text { Chronic }\end{array}$ & $\begin{array}{c}\text { TH } \\
\text { FI } \\
\text { Two-way information } \\
\text { exchange between TH and FI }\end{array}$ & $\begin{array}{c}\text { T-CBT } \\
1 \mathrm{~h} \text { telephone assessment } \\
30-45 \mathrm{~min}, 1 \times / \mathrm{w}, 7 \mathrm{w}, \mathrm{FU} 3 \text { and } 6 \mathrm{~m} \\
\text { By TH } \\
\text { Education }- \text { Knowledge of treatment } \\
\text { Cognitive-behavioral therapy } \\
\text { PE } \\
20-60 \mathrm{~h}-2 \times / \mathrm{w} \\
\text { By FI } \\
\text { General exercise therapy }\end{array}$ \\
\hline
\end{tabular}


Table 2. Cont.

\begin{tabular}{|c|c|c|c|c|c|}
\hline No. & Author, Year \& Country & Intervention Name & Target Population & Collaboration & Content and Intensity Intervention \\
\hline 34 & $\begin{array}{l}\text { 1: Bennell et al., (2012) [64] } \\
\text { 2: Hinman et al., (2015) [65] } \\
\text { 3: Bennell et al., (2017) [63] } \\
\text { Australia }\end{array}$ & $\begin{array}{l}\text { Physiotherapy plus telephone } \\
\text { coaching }\end{array}$ & $\begin{array}{l}\text { Patients with knee OA } \\
\text { Subacute and chronic }\end{array}$ & $\begin{array}{c}\text { TH } \\
\text { TC } \\
\text { Written information exchange } \\
\text { between the TC and TH } \\
\text { occurred after each session. }\end{array}$ & $\begin{array}{c}\text { Physical therapy program } \\
30 \mathrm{~m}, 5 \times, 6 \text { months } \\
\text { Specific exercise therapy } \\
\text { General exercise therapy } \\
\text { Information booklet } \\
\text { Education-Knowledge of disease prevention and } \\
\text { ergonomics } \\
\text { Telephone coaching } \\
6-12 \times, 6 \text { months } \\
\text { Cognitive-behavioral therapy }\end{array}$ \\
\hline
\end{tabular}

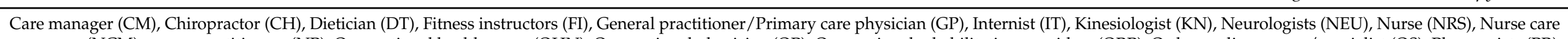

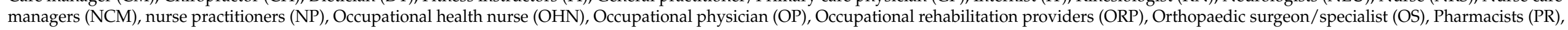

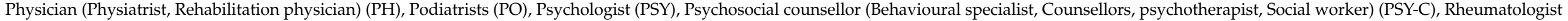

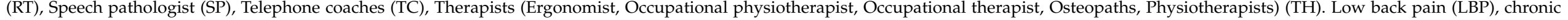
musculoskeletal pain (CMP), osteoarthritis (OA), rheumatoid arthritis (RA). 
Furthermore, six interventions existed of a collaboration between primary care and secondary or tertiary care [48-53]. Two of these interventions were between a rehabilitation department and primary care $[48,51]$. Examples of these collaborations were a GP in primary care with a $\mathrm{TH}$, orthopedic surgeon/specialist (OS), or NP in secondary or tertiary care. Collaborations between GPs and extensive rehabilitation teams, consisting of a nurse (NRS), PSY, TH, or PSY-C, and OS. Four interventions existed of an interdisciplinary assessment. In one study, assessment and follow-up were performed by an HCP in secondary care, a TH and a patient via video-conferencing (due to long-distance) [53]. Rothman et al. evaluated a collaboration in assessment and giving advice between a GP and at least three HCPs in secondary or tertiary care [52]. Two of these interventions consisted of an interdisciplinary assessment followed by treatment $[48,51]$. The other two interventions consisted of an interdisciplinary treatment without an interdisciplinary assessment $[49,50]$.

Additionally, one intervention was applied in an interdisciplinary pain clinic in primary care with collaboration in primary care, as well as between primary care and secondary and tertiary care [54]. THs who usually work in both primary care and secondary/tertiary care settings delivered the treatment in this interdisciplinary pain clinic in primary care, consisting of specific exercise therapy, medication management, and education.

Two interventions were a collaboration between primary care and social care $[55,56]$. Here, the teams consisted of several THs, a PH, and a case manager. Together, they performed a team assessment and, during the treatment and follow-up meetings, combinations of the HCPs involved delivered the treatment. In both treatments, workplace interventions were included, aimed at a return to work.

In addition, two interventions consisted of a collaboration between primary care, secondary/tertiary care, and social care [57,61]. These extensive interventions also involved the patients' medical specialists during workplace interventions, in addition to THs, GPs, and occupational physicians (OPs). While both interventions had many similarities, the recruitment of the study populations differed. In the studies of Steenstra et al. and Anema et al., the participants were recruited by the OP, while in the studies of Lambeek et al., the recruitment was by the PHs of the outpatient clinics of participating hospitals [57,59-61].

Finally, two interventions existed of a collaboration between primary care and communitybased initiatives [62,66]. In the intervention of McBeth et al., \& Bee et al., the TH delivered the $\mathrm{CBT}$, and the fitness instructor (FI) from a community-based initiative gave the general exercise therapy $[62,66]$. The intervention of Bennell et al., and Hinman et al. comprised a physical therapy program delivered by the TH in primary care, CBT by the telephone coach (TC), and an information booklet for education about the disease $[64,65]$.

\subsection{Quadruple Aim Outcomes}

An overview of the Quadruple Aim outcomes for each intervention is presented in Table 3. After data extraction from the included studies, it became evident that the interventions, outcome measures, and study designs were too heterogeneous to justify meta-analysis in the included studies. Therefore, narrative analyses were conducted. 
Table 3. Overview of study designs, study outcomes, and results based on the Quadruple Aim.

Author \& Year

Study Date
Study Outcomes

In primary care

Randomized trial design

Calner et al.

$2011-2014$

RCT

* Pain intensity (100-mm Visual Analogue Scale)

* Pain-related disability (Pain Disability Index)

$n=\mathrm{I}: 60, \mathrm{C}: 49$

Health-related quality of life (36-item Short-Form Health Survey)

Costs

* Work-related aspects and behavior § (Work Ability Index (7-49)) * Working percentage $\S$

$2 \quad$ Chelimsky et

al., (2013) [71]

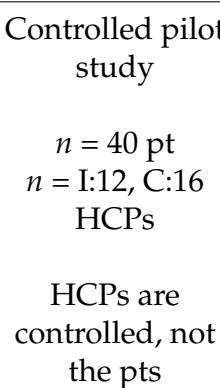

Results

$$
\text { Health }
$$

* Pain intensity (0-10 Numeric Rating Scale)

* Pain qualities (Short-Form McGill Pain Questionnaire)

* Physical functioning; measured with:

Multidimensional Pain Inventory Interference Scale - Brief Pain Inventory

- Multidimensional Health Locus of Control Scale

* Emotional functioning; measured with

$$
\text { - Back Depression Inventory }
$$$$
\text { - Profile of Mood States }
$$

Experienced quality of care by patients

* Participant ratings of global improvement and satisfaction with treatment; measured with:

- Patient Global Impression of Change

- Treatment helpfulness questionnaire

- Facilitation of patient involvement in care

Satisfaction with work by HCPs

* Experiences with work $¥$ (24-item physician perspectives questionnaire)

$$
\text { - Knowledge }
$$

Diagnosis/Managemen

- Treatment Comfort

- Treatment Satisfaction

$$
\text { - Use of Referrals }
$$

* Interview regarding: MD functional approach, Patient functional approach,

Enabling self-management, Assessing patient mood, Assessing patient sleep,

Comfort with use of medication 
Table 3. Cont.

Author \& Year

Study Design \& N

(2018) [77]

Study Date

Protocol

Randomized

pragmatic tria

Intended $n=85$

pt in clusters
Study Outcomes

Health

* Pain, Enjoyment, General Activity (PEG) § (3-item measure based on Short Form of the Brief Pain Inventory)

* Pain-related disability (Roland Morris Disability Questionnaire)

$$
\text { Costs }
$$

* Healthcare utilization (opioids dispensed, both aggregated and disaggregated

primary care contact, use of specialty pain services, inpatient services related to pain, and overall outpatient utilization

Experienced quality of care by patients

* Patients' satisfaction with their primary care services (one question)

* Satisfaction with overall pain-related services provided by the health plan (one question 
Table 3. Cont

Author \& Year

al. (2008) [67

2: Dobscha et
al., (2009) [72]

2006-2007 findings)

I: $187 \mathrm{pt}$,

$20 \mathrm{HCP}$

C: $214 \mathrm{pt}$,

$22 \mathrm{HCl}$

2: RCT $n=\mathrm{I}: 187, \mathrm{C}: 214$
Quality of life (EuroQoL-5D)

* Pain-related function/disability § (Roland Morris Disability Questionnaire)

* Pain severity (Chronic Pain Grade Severity subscale)

* Depression severity (Patient Health Questionnaire)

*Comorbidity (Chronic Disease Score (RxRisk-V [pharmacy data])

* Readiness for change (modeled after Epler)

* Global Impression of Change

$$
\text { Costs }
$$

* Opioid prescriptions (number, type, doses, duration)

* Use of adjuvant pain medications

* Concurrent use of multiple short-acting opioids

* Utilization and costs (primary care, pain specialty, mental health/SUD specialty,

emergency, other ambulatory treatment visits, contact, inpatient days)

Experienced quality of care

* Global Care Satisfaction

* Survey of Health Experiences of Veterans (pain care, 1-item)

Work satisfaction by HCPS

* Pain management attitudes/behaviors items * Job satisfaction

* Provider helpfulness of intervention

$$
\text { 2: Health }
$$

* Quality of life (EQ-5D)

* Pain-related function/disability § (Roland Morris Disability Questionnaire)

* Pain intensity (Chronic Pain Grade Severity subscale)

* Depression severity (Patient Health Questionnaire)

$$
\text { * Global Impression of Change }
$$

$$
\text { Costs }
$$

* Opioid prescriptions (number, type, doses, duration)

* Use of adjuvant pain medications, use of multiple short-acting opioids

* Utilization and costs (Primary care, pain specialty, mental health/SUD specialty, emergency, other ambulatory treatment visit, and contacts; inpatient days)

Experienced quality of care by patients

$$
\text { * Global Care Satisfaction }
$$

0-1 y: -

$0-1$ y: +

$0-1 \mathrm{y}:+$

$0-1 \mathrm{y}:+$

0-1 y: +

0-1 y: +

$0-1 \mathrm{y}:-$

0-1 y: +/-

0-1 y: - 
Table 3. Cont.

Author \& Year

Gustavsson et

al., (2018) [69]

Study Date

2011-2013

Study Design \& N

Feasibility study

Pragmatic RCT

$n=\mathrm{I}: 15, \mathrm{C}: 17 \mathrm{pt}$

$n=7 \mathrm{HCPs}$

Study Outcomes

Health

* Health-related quality of life (EuroQoL-5D)

* Disability

* Pain intensity

* Pain catastrophizing

* Pain-related fear-avoidance

* Depression

* Anxiety

Costs

* Sickness absence

* Costs-utility

Experienced quality of care by patients

* Patients' satisfaction with treatment (Self-assessment questionnaire)

Satisfaction with work by HCPS

* Perceived usability of the program $¥$ (interview)

* Proficiency in applying the techniques and delivering of the intervention components $¥$ (interview)

6 Hansson et al.

(2010) [75]

RCT

$n=\mathrm{I}: 61, \mathrm{C}: 53$

Health

* Self-perceived health $₫$ (EuroQol-5D)

* Function lower extremities (one-leg rising from sitting to standing)

* Balance performance; measured with:

- standing one leg eyes open

- standing one leg eyes closed
Results

n.a. (only baseline results)
$1 \mathrm{y}:-$

$9 \mathrm{w}:+/-1 \mathrm{y}:+$

$9 \mathrm{w}:-1 \mathrm{y}:-$

$+$

$6 \mathrm{~m}:$ Index:-VAS: + $6 \mathrm{~m}$ : -

$6 \mathrm{~m}:-$

$6 \mathrm{~m}:+$ 
Table 3. Cont.

Intended $n=$

I:54, C:54

$=\mathrm{I}: 55, \mathrm{C}: 56$

Study Outcomes

Results

* Self-reported pain § (pain subscale of the Western Ontario and McMaster Universities Osteoarthritis Index)

* Physical functioning and stiffness (corresponding subscales of the Western Ontario and McMaster Universities Osteoarthritis Index)

* Pain intensity (0-10 Numeric Rating Scale)

* Health-related quality of life (RAND-36 item Health Survey and 15-dimensional Health-related Quality of Life)

* Life satisfaction (4-item Life Satisfaction)

* Kinesiophobia (Tampa Scale for Kinesiophobia)

* Catastrophizing (Pain Catastrophizing Scale)

* Depressive symptoms (Beck Depression Inventory)

* Global assessment of change

$$
\text { Costs }
$$

* Use of analgesics, topical pain medication (patient reports)

* Number of intra-articular injections

* Use of health services

* Number of sick-leave days

* Cost-effectiveness (QALY) 2: Health

* Self-reported pain § (pain subscale of the Finnish version of the Western Ontario and McMaster Universities Osteoarthritis Index)

* Physical functioning and stiffness (corresponding subscales of the Western Ontario and McMaster Universities Osteoarthritis Index)

* Pain intensity (0-10 Numeric Rating Scale)

${ }^{*}$ Health related quality of life (RAND-36 item Health Survey and 15-dimensional Health-related Quality of Life)

* Life satisfaction (4-item Life Satisfaction)

* Kinesiophobia (Tampa Scale for Kinesiophobia)

* Catastrophizing (Pain Catastrophizing Scale)

* Depressive symptoms (Beck Depression Inventory)

* Global assessment of change

* BMI (weight/length2)

Costs

* Pain medication

* Use of health services

* Number of sick-leave days
$3 \mathrm{~m}:-$

$3 \mathrm{~m}:-$

$3 \mathrm{~m}:-$

$3 \mathrm{~m}:-$

$3 \mathrm{~m}:-$

$3 \mathrm{~m}:-$

$3 \mathrm{~m}:-$

$3 \mathrm{~m}:-$

$3 \mathrm{~m}:-$

$3 \mathrm{~m}:-$ 
Table 3. Cont.

Author \& Year

1: Nordin et

al., (2016) [74]

Study Date

2011-2015

Study Design \& $\mathbf{N}$

1: RCT

$n=\mathrm{I}: 55, \mathrm{C}: 43$

2: Nordin et

al., (2017) [79]

2011-2015
Study Outcomes

1: Health

* Pain intensity II (100-mm Visual Analogue Scale)

Experienced quality of care by patients

* Patients' satisfaction with the intervention (2-items)

Costs

* Intervention characteristics

* Health care consumption

$$
\text { * Sick leave }
$$

2: Experienced quality of care by patients 9

* Experiences of patient participation in the rehabilitation and intervention

Theme: It's about me

- Take part in a flexible framework of own priority

- Acquire knowledge and insights

- Ways toward change

- Personal and environmental conditions influencing participation

Non-randomized trial designs

1: Dunstan et

al., (2007) [80

1: Pilot study

Uncontrolled

measures design

$n=30$

2: Dunstan et

al., (2014) [81]

2: Qualitative

design

$n=33$

\section{1: Health}

* Pain severity (0-10 Numeric Rating Scale)

* Mood (Depression, Anxiety, Stress Scales)

* Disability (Modified Roland Morris Disability Questionnaire)

* Catastrophizing (Pain Catastrophizing Scale)

* Fear-avoidance (Tampa Scale for Kinesiophobia)

$$
\text { Costs }
$$

* Paid work participation $¥$ (for any number of hours)

\section{2: Experienced quality of care by patients $\mathbb{I}$}

* How much the program helped them to manage their pain, become more active, and get back to work (5-point Likert-type scales)

* The helpfulness of each component of the program (5-point Likert-type scales)

* The quality of the psychologist's and physiotherapists' input (5-point Likert-type scales)

* Program improvements
Results

4 m:-1 y: -

$4 \mathrm{~m} \& 1 \mathrm{y}$ : intervention: +

$4 \mathrm{~m} \& 1 \mathrm{y}:$ own effort: -

no significance calculated 
Table 3. Cont

Author \& Year

Gurden et al.,

(2012) [82]

Study Date

2009-2010

Unconign \& $\mathbf{N}$

Uncontrolled

pilot study

$n=696$

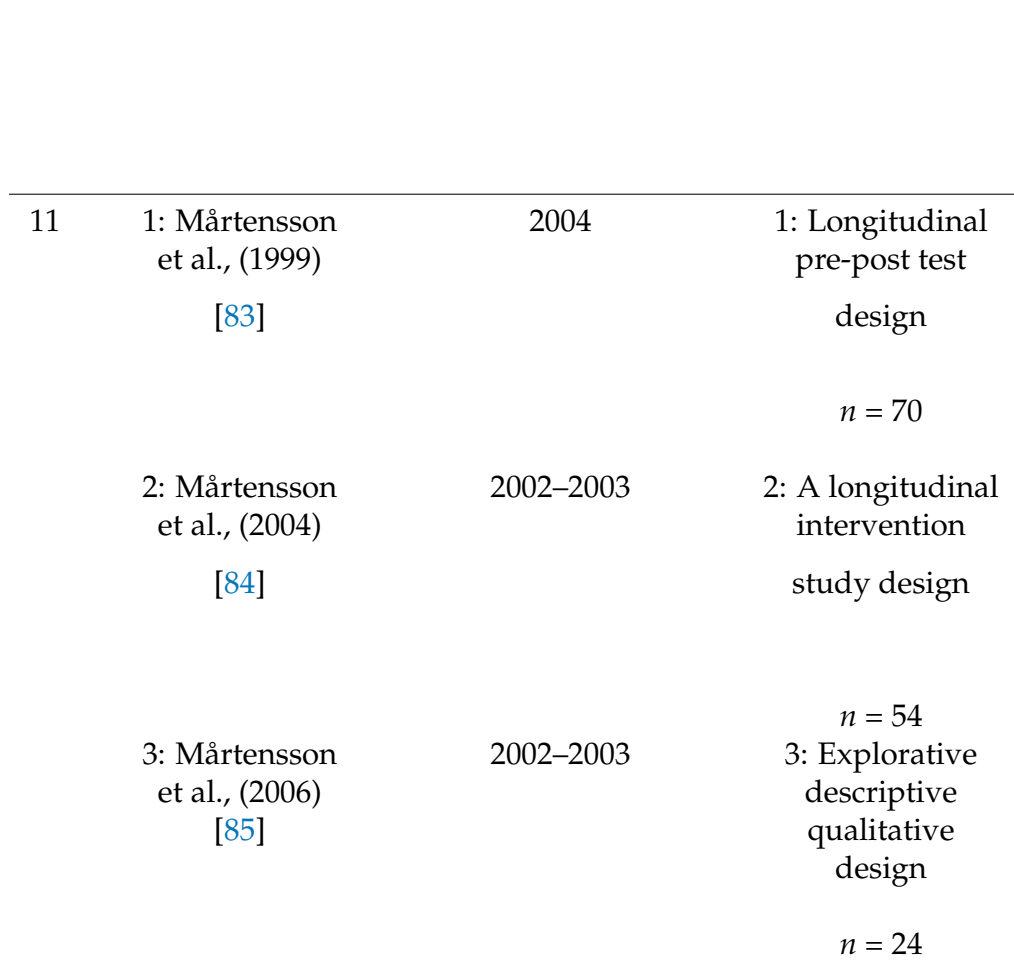

\section{Study Outcomes}

Health

* Back and neck pain $¥$ (Bournemouth Questionnaire)

Costs

* Medication usage

* Other healthcare utilization

* Work status

Experienced quality of care by patients

* a patient satisfaction with treatment scale (5-point scale)

* General well-being $¥$ (100-mm Visual Analogue Scale)

* Pain management ability (100-mm Visual Analogue Scale)

* Perceived complaints (100-mm Visual Analogue Scale)

* Influence of the intervention and perceived change due to treatment (Personality-

$$
\text { Physical-Cognitive) }
$$

$$
\text { 2: Costs }
$$

* Sick leave days II (Statistics register at the social insurance office)

* Doctor visits (Statistics register at the county council in question)

* Level of absenteeism due to occupational disability (Statistics register at the social

$$
\text { insurance office) }
$$

3: Experienced quality of care by patients $\S$

* Content, format, the group's role, the leader's role, and the participant's role$$
\text { - A place to which you belong }
$$

- Expectations of being regarded as a sick person

- The value of one's own contribution

$$
\text { - Reacting but not acting }
$$

Results

Baseline and discharge:

$+$

$+$

no results described $+$

$0 \mathrm{~m}-2 \mathrm{~m}:+0 \mathrm{~m}-2 \mathrm{y}:+$

$0 \mathrm{~m}-2 \mathrm{~m}:-0 \mathrm{~m}-2 \mathrm{y}:+2 \mathrm{~m}-2 \mathrm{y}$ $+$

$0 \mathrm{~m}-2 \mathrm{~m}:+0 \mathrm{~m}-2 \mathrm{y}:+$ Body awareness: + Other: -

$0 \mathrm{~m}-1 \mathrm{y}:-0 \mathrm{~m}-2 \mathrm{y}:+1 \mathrm{y}-2 \mathrm{y}:+$ $0 \mathrm{~m}-1 \mathrm{y}:+0 \mathrm{~m}-2 \mathrm{y}:+1 \mathrm{y}-2 \mathrm{y}:$ $+$

0 m-post: $+0 \mathrm{~m}-1 \mathrm{y}:+0 \mathrm{~m}-2$ y: +

+
+
-
+
- 
Table 3. Cont.

Author \& Yea

Schütze et al.,

(2014) [86]
Study Date

Study Design \& $\mathbf{N}$

Pilot study

Repeated

measures design

$n=12$
Study Outcomes

Health

* Risk of future disability (Örebro Musculoskeletal Pain Questionnaire)

* Low-back related functional disability (Oswestry Disability Questionnaire)

* Emotional functioning (Short form of the Depression, Anxiety, Stress Scales)

$$
\text { - Depression }
$$$$
\text { - Anxiety }
$$

* Present-moment awareness of actions, interpersonal communication, thought emotions, and physical state (Mindful Attention Awareness Scale)

$$
\text { * Catastrophizing (Pain Catastrophizing Scale) }
$$

${ }^{*}$ Health status and health-related quality of life II (36-item Short-Form Health

$$
\text { Survey) }
$$

Experienced quality of care by patients

* Patient satisfaction (Client Satisfaction Questionnaire)

Health

* Pain intensity (0-10 Numeric Rating Scale)

* Anxiety and depression (Hospital and Anxiety Depression Scale)

$$
\text { - Anxiety }
$$

$$
\text { - Depression }
$$

* Pain severity (Multidimensional Pain Inventory)

* Health-related quality of life; measured with:

-36-item Short-Form Health Survey, social function

- EuroQoL-5D, physical function Costs

* Sick-leave (Software-system "Swedestar")

* Consumption of opioids § (Software-system "Swedestar")
Results

$0 \mathrm{~m}-3 \mathrm{~m}:-0 \mathrm{~m}-6 \mathrm{~m}:-$

m-3 $m:+0 m-6$

0 m-3 m:-0 m-6 m: + $0 \mathrm{~m}-3 \mathrm{~m}:-0 \mathrm{~m}-6 \mathrm{~m}:-$

$0 \mathrm{~m}-3 \mathrm{~m}:+0 \mathrm{~m}-6 \mathrm{~m}:+$

0 m-3 m:-0 m-6 m: +

$0 \mathrm{~m}-3 \mathrm{~m}:+0 \mathrm{~m}-6 \mathrm{~m}:+$ All: $0 \mathrm{~m}-3 \mathrm{~m}:+0 \mathrm{~m}-6 \mathrm{~m}:$ 
Table 3. Cont.

Author \& Year

Tyack et al.,

(2013) [88]
Study Date

Study Design \& N

Longitudinal

cohort study

Intended $n=130$

\section{Study Outcomes}

Results

Health § (36-item Short-Form Health Survey)

* BMI (weight/length2)

* Waist circumference

Psychological distress (6-item Kessler)

Disease burden (self-report comorbidity measure)

* Comorbid conditions (self-report comorbidity measure)

* Perceived functional and structural social support (Medical Outcomes Study Social Support Survey)

Self-reported health perception (one question)

Costs

* Hospital utilization (number of days spent in hospital, and number of hospita admissions)

* Healthcare costs (Medicare and hospital utilization records)

15 Westman et

al., (2006) [89]
1994-1996

Cohort

$n=72$

Health

* Quality of life-life satisfaction $¥$ (10-items self-constructed questionnaire) * Intensity of pain and frequency (100-mm Visual Analogue Scale)

* Function (Disability Rating Index)

* Anxiety and depression (Hospital and Anxiety Depression Scale)

* Health profile assessment

Experienced quality of life by patients

* Patient satisfaction (three questions)

$$
\text { Costs }
$$

* Sick leave/Return to work (self-reported data)

* Job strain (11-items self-constructed questionnaire)
$0 \mathrm{~m}-1 \mathrm{y}:-0 \mathrm{~m}-5 \mathrm{y}:+$ $0 \mathrm{~m}-1 \mathrm{y}:+0 \mathrm{~m}-5 \mathrm{y}:+$ $0 \mathrm{~m}-1 \mathrm{y}:-0 \mathrm{~m}-5 \mathrm{y}:+$

$0 \mathrm{~m}-1 \mathrm{y}:-0 \mathrm{~m}-5 \mathrm{y}:+$ $0 \mathrm{~m}-1 \mathrm{y}:+0 \mathrm{~m}-5 \mathrm{y}:+/-$ $+1$ $0 \mathrm{~m}-1 \mathrm{y}:+0 \mathrm{~m}-5 \mathrm{y}:-$ 
Table 3. Cont.

Author \& Yea

Westman et

al., (2010) [90]

Study Date

1998-2000

Study Design \& N

Trial with

control group

$n=\mathrm{I}: 59, \mathrm{C}: 52$
Study Outcomes

Health

* Health-related quality of life $¥$ (36-item Short-Form Health Survey)

* Coping (Coping strategies questionnaire)

* Catastrophizing (Pain Catastrophizing Scale)

* Fear of movement (Tampa Scale for Kinesiophobia)

* Psychosomatic symptoms

Costs

* Work capacity/sick leave (reported by patients)

* Job strain (11-items, self-constructed questionnaire)

Health care utilization (how many visits ( 0 to 10 ) during the past 12 months)

Physiotherapist

Naprapath or chiropractor

Drug consumption (one question)

Qualitative designs

1: Description of

1: n.a.

1: Dorflinge

[91]

2: Purcell et

al., (2018) [25

2015-2016

intervention

2: Mixed

methods trial

2: Work satisfaction by HCPs

* Perspectives on the perceived effectiveness of the chronic pain care provided to

$$
\text { their patients } ¥
$$

Interview: $n=6$

Questionnaire:

$n=65$

their patients $*$

Confic

(questionnaire)

* Burnout measure (1 item, based on Maslach Burnout Inventory) 
Table 3. Cont.

Author \& Year

(2016) [65]

2: Lovo et al.

2014-2015

(2019) [92]
Study Date

Intended $n=$

I:20 C:20

$\begin{array}{cc}\text { 2: Lovo et al., } & \text { 2: Qualitative } \\ \text { (2019) [92] } & \text { design } \\ & \text { Questionnaire: } \\ & n=19 \mathrm{pt} \\ \text { Interview: } & n=2 \mathrm{HCP}, \\ n=6 \mathrm{pt}\end{array}$

*Self-perceived function § (Modified Oswestry Disability Questionnaire)

$$
\text { * Pain intensity (0-10 Numeric Rating Scale) }
$$

${ }^{*}$ Quality of life/general health status (EuroQoL-5D)

$$
\text { Costs }
$$

* Costs (self-report diaries: intervention/treatment costs, work status, absenteeism

and disability days related to back pain, health service use, other pain-related costs, and costs from participation in the study Experienced quality of care by patients

* Patient satisfaction (a modified version of the Visit Specific Satisfaction Instrument)

$$
\text { Work satisfaction by HCP }
$$

* Satisfaction of HCPs with intervention (semi structured interview)

2: Experienced quality of care by patients

* Patient satisfaction (a modified version of the Visit Specific Satisfaction Instrument a space for comment, and semi-structured interviews)

$$
\text { Work satisfaction by HCPs }
$$

* Satisfaction of HCPs with PT-delivered telehealth assessments $\S$

$$
\text { (semi structured interview) }
$$

$$
\text { - Access to care }
$$

- Effective interprofessional practice

- Enhanced clinical care

$$
\text { - Technology }
$$

\begin{tabular}{ccc}
\hline $19 \quad$ Pietilä & - & Qualitative \\
interviews & \\
Holmner et al., & &
\end{tabular}

Experienced quality of care by patients

* Experiences of MMR § (interview) - from discredited towards obtaining redress

- from uncertainty towards knowledge

- from loneliness towards togetherness 
Table 3. Cont

Author \& Year

\section{Stenberg et al.}

(2016) [94
Study Date

Study Design \& $\mathbf{N}$

design

$n=14$ $\begin{array}{cc}\text { Study Outcomes } & \text { Results }\end{array}$

$\begin{array}{ccc}\text { Study Outcomes } & \text { Results }\end{array}$

* Benefits and drawbacks of including patients in MMR

* Types of patients in MMR

* Factors that facilitate or impede conduct of MMR

* Professional views on MMR

* Teamwork experiences

Select patients for success

- Multilevel challenge

Ethical dilemmas

-Considering what is a good result

21 1: Sundberg et 2003-2006 1: Qualitative

al., (2007) [76]

study design

1: Barriers and facilitators: Notes of research group meetings $(n=40)$ and

field notes from seminars and lectures about results from the development

$$
\text { and implementation phases }
$$

2: Sundberg et

2: Feasibility

study

Pragmatic RCT

${ }^{*}$ Health-related quality of care $§$ (36-item Short-Form Health Survey)

* Disability (0-10 Numeric Rating Scale)

* Stress (0-10 Numeric Rating Scale)

$n=\mathrm{I}: 36, \mathrm{C}: 27$

* Well-being (0-10 Numeric Rating Scale)

* Days in pain (0-10 Numeric Rating Scale) Costs

* Use of analgesics (0-10 Numeric Rating Scale)

* Use of health care (0-10 Numeric Rating Scale)

$16 \mathrm{w}:$ All subscales:

$16 \mathrm{w}:-$

$16 \mathrm{w}:-$

$16 \mathrm{w}:-$

16 w: -

$16 \mathrm{w}:-$

$16 \mathrm{w}:-$ 
Table 3. Cont.

Between primary care and secondary or tertiary care

\begin{tabular}{lllll} 
& \multicolumn{3}{c}{ Randomized trial designs } \\
\hline 22 & Haldorsen et & - & RCT & Health
\end{tabular}

$n=\mathrm{I}: 312 ; \mathrm{C}: 157$ al., (1998) [50]

* Quality of life (6-items, self-constructed questionnaire)

$1 \mathrm{y}:+/$ n.d.

* Pain intensity (drawing test and 100-mm Visual Analogue Scale)

* Amount of pain caused by daily activities (Activity Discomfort Scale)

* Subjective health (Ursin's Health Inventory)

* Anxiety (Spielberger State Trait anxiety Scale)

* Psychological distress (brief version of the Hopkins Symptom Check List)

* Health locus of control (Multidimensional Health Locus of Control Scale)

* Physiotherapy examination (functional ability, movement, relaxation ability, pain aerobic capacity test, practical skills)

$$
\text { Costs }
$$

* Return to work after 12 months $\S$

* Subjective work ability (Graded Reduced Work Ability scale)

$1 \mathrm{y}:+$

23 Rothman et
al., (2013) [52

2001-2004

RCT

$n=\mathrm{I}: 91, \mathrm{C}: 91$

* Pain intensity \& (100-mm Visual Analogue Scale)

* Depressive symptoms (Zung Self-Rating Depression Scale)

* Stress-related symptoms (Stress and Crisis Inventory)

* Quality of life (36-item Short-Form Health Survey)

* Pain related disability (Oswestry Disability Index)

$$
\text { Costs }
$$

* Work ability (Swedish government insurance company)

$1 \mathrm{y}:+/-$

$1 \mathrm{y}:+$

$1 \mathrm{y}:-$

$1 \mathrm{y}:+$

$1 \mathrm{y}:+$

$1 \mathrm{y}:+/-$

$1 \mathrm{y}:-$

$15 \mathrm{~m}:-$

$15 \mathrm{~m}:-$

$15 \mathrm{~m}:+/$

$15 \mathrm{~m}:-$

$15 \mathrm{~m}:+$

Experienced quality of care by patient

* Patient satisfaction with assessment (study-specific questionnaire)

$15 \mathrm{~m}:+$ Health

$\mathrm{RCT}$

* Disease activity § (Disease Activity Score-28)

[53]

$n=\mathrm{I}: 31, \mathrm{C}: 23$
* Quality of Life (EuroQoL-5D)

* Patient's global function score (100-mm Visual Analogue Scale, global function)

Experienced quality of care by patients

* Satisfaction (9-item visit-specific satisfaction questionnaire)
$9 \mathrm{~m}:-$

$9 \mathrm{~m}:-$

$9 \mathrm{~m}:-$

$9 \mathrm{~m}:-$ 
Table 3. Cont

\section{Author \& Year}

Study Date

Study Design \& N

2006-2007

al., (2010) [48]

Prospective

cohort

$n=29$

26 Claassen et al.,

(2018) [49]

2015-2016

Observational

pilot study

$n=107$
Study Outcomes

Results

Non-randomized trial desions

Health

* Pain intensity (0-10 Numeric Rating Scale)

* Pain interference $¥$ (Pain Interference Questionnaire)

I-4: 0 m-discharge: +

I-4: 0 m-discharge: +

\begin{tabular}{|c|c|c|c|c|c|}
\hline & (2018) [49] & & $\begin{array}{l}\text { pilot study } \\
\qquad n=107\end{array}$ & $\begin{array}{c}\text { * BMI (weight/length2) } \\
\text { * Pain and limitations in functional activities (Western Ontario and McMaster } \\
\text { Universities Osteoarthritis Index, pain and physical functioning subscales) } \\
\text { * Illness perceptions (Brief illness Perception Questionnaire) } \\
\text { * Physical activity (Short Questionnaire to Assess Physical Activity) } \\
\text { Costs } \\
\text { * Healthcare Utilization } ¥ \text { (self-constructed questionnaire and patient diary pain } \\
\text { medication, total number of contacts) } \\
\text { Experienced quality of care by patients } \\
\text { * Patient satisfaction (1-item, with satisfaction with course) }\end{array}$ & $\begin{array}{l}0 \text { m-3 m: - } \\
0 \text { m-3 m: - } \\
0 \text { m-3 m: + } \\
0 \text { m-3 m: - } \\
0 \text { m-3 m: + }\end{array}$ \\
\hline 27 & $\begin{array}{l}\text { Plagge et al., } \\
\text { (2013) [51] }\end{array}$ & - & $\begin{array}{l}\text { Retrospective } \\
\text { study } \\
\quad n=30\end{array}$ & $\begin{array}{c}\text { Health } \\
\text { * Pain severity and interference (Chronic Pain Grade) } \\
\text { * Pain catastrophizing (Pain Catastrophizing Scale) } \\
\text { * Fear avoidance (Tampa Scale for Kinesiophobia) } \\
\text { * Depressive symptoms (Patient Health Questionnaire) } \\
\text { * Quality of life II (Center for Disease Control Health-Related Quality of Life } \\
\text { Measure) } \\
\text { * Satisfaction with life (Satisfaction with Life Scale) }\end{array}$ & $\begin{array}{c}0 \text { m-post: All: + } \\
0 \text { m-post: + } \\
0 \text { m-post: + } \\
0 \text { m-post: }+ \\
0 \text { m-post: All: + } \\
0 \text { m-post: + }\end{array}$ \\
\hline \multicolumn{6}{|c|}{ In primary care and between primary care and secondary or tertiary care } \\
\hline & & & & $\begin{array}{l}\text { Experienced quality of care by patients } \\
\text { * Satisfaction of patients with their health care }\end{array}$ & $2 \mathrm{~m}:+$ \\
\hline
\end{tabular}


Table 3. Cont.

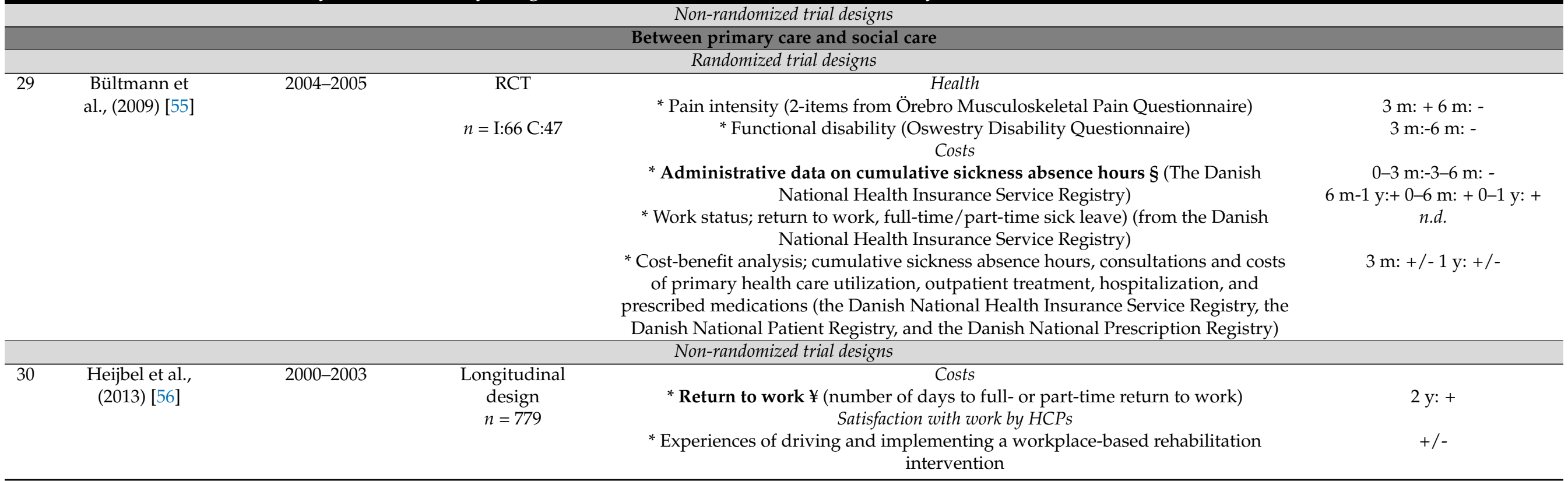


Table 3. Cont

Author \& Year

Study Date

Study Design \& N

Study Outcomes

Results

Between primary care and secondary or tertiary care and social care

al., (2007) [58

$2005-2009$

1: Protocol RCT

Intended $n=$

$\mathrm{I} \cdot 65, \mathrm{C} \cdot 65$

* Pain intensity (10-point Visual Analogue Scale)

* Functional status (Roland Morris Disability Questionnaire)

Quality of life (EuroQoL-5D)

Costs

* Return To Work § (sick leave in calendar days during study until full return

to work in own or other work, for at least 4 weeks without recurrence)

$$
\text { * Total duration of sick leave }
$$

* Direct (non)-medical costs (diaries)

Experienced quality of care by patients

* Patient satisfaction (Patient Satisfaction with Occupational Health Services

2: Lambeek et

2005-2009

2: RCT

al., (2010) [59]

$n=\mathrm{I}: 66, \mathrm{C}: 68$

3: RCT

3: Lambeek et

2005-2009

al., (2010) [60]

$$
\text { * Total duration of sick leave }
$$

$$
\begin{gathered}
\text { Questionnaire } \\
\text { 2: Health }
\end{gathered}
$$

Pain intensity (10-point Visual Analogue Scale)

* Functional status (Roland Morris Disability Questionnaire)

Costs

* Return To Work $§$ (sick leave in calendar days during study until full return to work in own or other work, for at least 4 weeks without recurrence)$$
\text { 3: Costs }
$$

* Duration until sustainable Return To Work § (sick leave in calendar days during

study until full return to work in own or other work, for at least 4 weeks without

$$
\text { recurrence) }
$$

* Direct (non)-medical costs (diaries)

- Total costs and indirect costs

$$
\begin{aligned}
& \text { - Total direct costs } \\
& \text { Cost-effectiveness } \\
& \text { - Cost-benefit }
\end{aligned}
$$

$3 \mathrm{~m}:-6 \mathrm{~m}:-1 \mathrm{y}:-$

$3 \mathrm{~m}:-6 \mathrm{m:-1} \mathrm{y}:+$

$1 \mathrm{y}:+$

$1 \mathrm{y}:+$

$1 \mathrm{y}:+$

$1 \mathrm{y}:+$

$1 \mathrm{y}:-$

$1 \mathrm{y}:+/-$

$1 \mathrm{y}:+$ 
Table 3. Cont.

Author \& Year

\section{1: Steenstra et}

al., (2003) [61]

2: Anema et

al., (2007) [57]
Study Date

Study Design \& $\mathbf{N}$

Study Date 2002

1: Protocol RCT

Intended $n=$

I:100, C:100

2000-2002
2: Pragmatic
RCT
$n=\mathrm{I}: 27, \mathrm{C}: 85$

Study Outcomes

Results

* Functional status (Roland Morris Disability Questionnaire)

* Pain intensity (10-point Visual Analogue Scale)

* Kinesiophobia (Tampa Scale for Kinesiophobia)

* Fear of movement (Fear Avoidance Beliefs Questionnaire)

$$
\text { * Coping (Pain Coping Inventory Scale) }
$$$$
\text { Costs }
$$

* Return to work in the year after the first day of sick leave $\S$

* Workers use of pain medication and use of medical and alternative medical

resources and general health status (EuroQoL-5D)

Experienced quality of care by patients

* Patient satisfaction (short version Patient Satisfaction with Occupational Health Services Questionnaire)

$$
\text { 2: Health }
$$

* Functional status (Roland Morris Disability Questionnaire)

* Pain (10-point Visual Analogue Scale)

$1 \mathrm{y}:-$

$1 \mathrm{y}:-$

Costs 
Table 3. Cont.

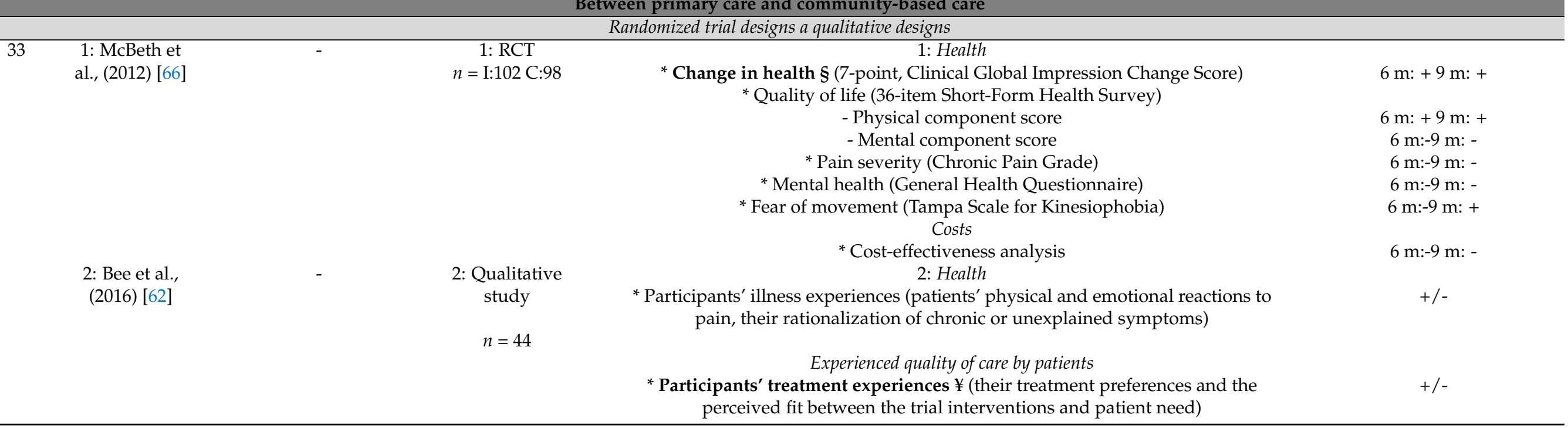


Table 3. Cont.

Author \& Year

Study Date

Study Design \& N

Study Outcomes

Results
Pragmatic RCT

Intended $n=$ I:67, C:67

2: Process

2: Hinman et

al., (2016) [65]

3: Bennell et

al., (2017) [63] evaluatio

$n=6 \mathrm{pt}$

$n=14 \mathrm{HCP}$

2012-2015

3: Pragmatic

RCT

$n=\mathrm{I}: 84 \mathrm{C}: 84$
* Average pain in the past week § (11-point Numeric Rating Scale)

* Physical function in past $48 \mathrm{~h}$ (Western Ontario and McMaster Universities

Osteoarthritis Index, physical function subscale)

* Global rating of change

Change in pain (7-point ordinal scale)

* Change in physical functioning (7-point ordinal scale)

* Physical activity (Physical Activity Scale for the Elderly, Active Australia Survey, stepping duration and steps per day over 7 consecutive days)

* Health-related quality of life (Assessment of Quality of Life Instrument version 2)

* Mood (Arthritis Impact Measurement Scale Version 2)

* Emotional state (Depression, Anxiety and Stress Scale)

* Fear of injury (Brief Fear of Movement Scale)

* Symptom severity (Patient Health Questionnaire)

* Coping (Coping Strategies Questionnaire)

* Catastrophizing (Pain Catastrophizing Scale)

2: Experienced quality of care by patients

Satisfaction with care by HCPs

- Theme 1: genuine interest and collaboration

- Theme 2: information and accountability

- Theme 3: program structure

- Theme 4: roles and communication in teamwork

$$
\text { 3: Health }
$$

* Knee pain intensity $\S(11$-point Numeric Rating Scale)

* Physical function in the previous $48 \mathrm{~h} \S$ (Western Ontario and McMaster Universities Osteoarthritis Index)

* Pain on walking in the past week (11-point Numeric Rating Scale)

* Pain (Western Ontario and McMaster Universities Osteoarthritis Index)

${ }^{*}$ Health related quality of life (Assessment of Quality of Life Instrument version 2)

* Physical activity (stepping duration and steps per day over 7 consecutive days) Costs

* Number of physiotherapy visits
$+$

$+1-$

$6 \mathrm{~m}:-1 \mathrm{y}:-18 \mathrm{m:}-$ $6 \mathrm{~m}:-1 \mathrm{y}:-18 \mathrm{~m}:-$

$6 \mathrm{m:-1}$ y:-18 m:$6 \mathrm{~m}:-1 \mathrm{y}:-18 \mathrm{~m}:-$ $6 \mathrm{~m}:-1 \mathrm{y:}-18 \mathrm{~m}:-$ $6 \mathrm{~m}:+1 \mathrm{y}:+18 \mathrm{~m}:+$

No comparison between groups

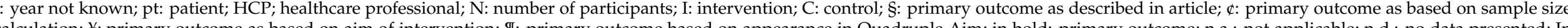

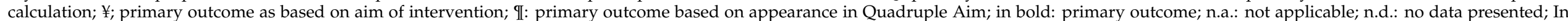

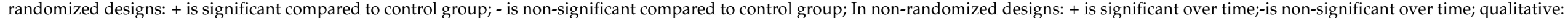

+ only positive opinions mentioned; - only negative opinions mentioned; +/- positive as well as negative opinions mentioned. Articles without results are described in grey. 
Among the 49 articles, 19 randomized trials, 12 non-randomized studies, 7 qualitative studies, 7 study protocols, 1 description of an intervention, 2 studies with a population with mixed diagnoses, and 1 study regarding barriers and facilitators, were found. Thirty-nine articles had at least one of the Quadruple Aim outcomes as the primary outcome: 18 articles described health outcome measures, 12 described cost outcome measures, 4 described quality of care experienced by patients, and 5 articles describe work satisfaction for HCPs. Hinman et al. described quality of care experienced by patients and HCP work satisfaction as the combined primary outcome [65]. Most studies measured more than one Quadruple Aim outcome, but only two interventions intended to assess all Quadruple Aim outcomes. Dobscha et al. measured all Quadruple Aim outcomes but presented only the baseline results [67]. Bath et al. described all Quadruple Aim outcomes in the protocol article, but not all results are published yet [68].

The outcomes of Dobscha et al., and Gustavsson et al., were only described as baseline measurements for an RCT $[67,69]$. From the remaining articles, comprising study protocols, description of the intervention, studies with mixed diagnoses, and the study regarding barriers and facilitators, no outcomes could be extracted [25,48,51-54,58,60,71,72,83-85,96]. These studies were used for descriptions of interventions in Table 2.

\subsubsection{Within Primary Care-Randomized Trial Designs}

The most frequently presented outcome measure among randomized trial designs was pain intensity (five studies [70-74]). For Helminen et al., the maximum follow-up time was three months, resulting in a non-significant difference between intervention and control groups [73]. Four studies reported outcomes at one-year follow-up: two reported a significant improvement (50\%) [71,72], while two reported no significant improvement (50\%) [70,74]. Pain intensity scores were measured with the 100-mm Visual Analogue Scale (VAS), the Chronic Pain Grade Severity subscale, or the 0-10 numerical pain rating scale (NPRS). Health-related quality of life (HRQoL) was measured in five studies. The improvement on the VAS score at six months of Hansson et al. (2010) was significantly different between intervention and control groups (20\%) [75]. In the other four studies $(80 \%)$, HRQoL was not different between the groups at three, four, or twelve months, as measured with the RAND-36, Short Form-36, and EQ-5D questionnaires [70,72,73,76].

Outcomes regarding sick leave/working days or medication prescription and use were most often measured for the Quadruple Aim of health care costs (five studies). At four-month follow-up, Calner et al. found positive and negative changes at the different levels of working percentages for the number of working participants in the intervention group compared to the control group [70]. Regarding sickness absence, Gustavsson et al. found no significant change in absence at one-year follow-up [69]. Additionally, Helminen et al. found no significant difference in the number of sick-leave days at three-month followup between the intervention and control groups $[73,76]$. They also found no difference in medication use at three-month follow-up between the control and intervention group, just like Sundberg et al. at four-month follow-up [73,76]. Dobscha et al. found a significant difference in opioid prescriptions at one-year follow-up but non-significant differences in the use of adjuvant pain medications between intervention and control groups [72].

Quality of care experienced by patients was measured in two studies. On a selfassessment questionnaire measuring the quality of care completed by patients, Chelimsky et al. found a significant result at one year for the facilitation of patient involvement in care, though no differences at nine weeks and one year were seen by Gustavsson et al. [69,71].

Both of these studies also examined HCP satisfaction with the care they delivered. At one-year follow-up, HCPs providing the interventions rated their work (significantly) more positively than did HCPs in the control conditions.

\subsubsection{Within Primary Care-Non-Randomized Study Designs}

All included studies had a longitudinal design (follow-up after intervention), but only two studies included a control condition. All seven interventions evaluated health out- 
comes. Pain intensity was only reported in two studies, with mixed results at one year and significant pain decreases at five years in both studies [87,89]. In four studies, including one protocol, HRQoL was the primary outcome, and in one study, it was the secondary outcome. At three months, six months, one year, and five years, significant changes were found. HRQoL was measured with the Short Form-36 or a self-constructed questionnaire $[86,87,90]$. Three studies reported no change in quality of life at one- and three-year follow-ups, as measured with the Short Form-36, EQ-5D, or self-constructed questionnaire $[87,89,90]$.

Costs were evaluated in six interventions, with sick leave or paid work participation as the most frequently reported measurement. In Dunstan et al. and Westman et al., the changes in paid work participation and sick leave were non-significant ( $40 \%$ of the studies) at six-months and three-year follow-up, respectively [80,90]. Stein et al. and Mårtensson et al. found at one- and two-year follow-ups significant changes, compared with baseline assessment $(40 \%$ of the studies) [84,87]. Westman et al. reported mixed results $(20 \%$ of the studies) for both sick leave and return to work [89]. Stein et al. and Westman et al. found no significant changes at one- and three-year follow-ups for opioids and drug consumption $[87,90]$. On the other hand, Gurden et al. found a significant decrease in medication usage after discharge [82].

Three studies found significant positive (100\%) results for quality of care experienced by patients $[82,86,89]$.

None of these interventions evaluated HCPs' satisfaction with the care they delivered.

\subsubsection{Within Primary Care-Qualitative Designs}

None of the six qualitative designs within the primary care interventions evaluated changes in health or costs.

Quality of care experienced by patients was assessed on different items by three studies, with mixed results $[79,81,93]$. For example, Dunstan et al. found that patients made both positive and negative points about the usefulness of the program for managing their pain, helping them to become more active and to get back to work, but expressed only positive views about the quality of treatment by HCPs. [81]. Additionally, one study found clear positive results (atmosphere, environment, value of one's contribution) and negative results (expectations of a sick person, reacting but not acting, awareness and integration) on various items [85]. Lovo et al. reported positive results for the quality of care, measured with both qualitative questionnaires and interviews [92].

Lovo et al. evaluated HCP work satisfaction, reporting overall positive results regarding access to care, effective inter-professional practice, and enhanced clinical care [92]. Only technology (telehealth) was scored less positively.

\subsubsection{Between Primary Care and Secondary or Tertiary Care-Randomized Trial Designs}

All three randomized trial designs of the interventions combining primary care and secondary or tertiary care measured health outcomes [50,52,53]. Rothman et al. reported a non-significant difference between the intervention and control groups at 15-month followup for the primary outcome of pain intensity [52]. In the study of Taylor-Gjevre et al., the difference between intervention and control groups in disease activity at a nine-month follow-up was also non-significant [53]. All three studies measured HRQoL but different measurement instruments were used (EQ-5D, Short Form-36, and a self-constructed questionnaire). In two studies, significant results were found on some questionnaires' subscales, with non-significant results on other subscales, at one-year and 15-month follow-ups, while one study found non-significant results at a nine-month follow-up.

Cost outcomes were measured by Haldersen et al., and Rothman et al. [50,52]. Changes in return to work after 12 months did not differ between groups, while changes in ability to work did at a 15-month follow-up.

Rothman et al., and Taylor-Gjevre et al. measured experienced quality of care by patients with questionnaires [52,53]. Although no differences were found at nine months, at the 15-month follow-up, the intervention group rated quality of care experienced higher 
than did the control group. HCP work satisfaction was not measured in any of these three studies.

\subsubsection{Between Primary Care and Secondary or Tertiary Care-Non-Randomized Trial Designs}

Health outcomes were assessed with less widely used outcome measures by all three non-randomized trial designs $[48,49,51]$. Burnham et al. had pain interference as the primary outcome, which was significant after treatment discharge in the cohort study [48]. Plagge et al. found significant changes in all domains of HRQoL post-intervention [51]. Claassen et al. found significant improvement only in illness perceptions after three months, whereas non-significant differences were found for BMI, pain, and limitations in functional activities, and physical activity after three months [49].

The study of Claassen et al. was the only one measuring the health care costs and quality of care experienced by patients, goals of the Quadruple Aim; both had significant results at the three-month follow-up [49].

None of the three included studies measured HCP work satisfaction.

\subsubsection{In Primary Care and between Primary Care and Secondary or Tertiary Care-Randomized Trial Design}

Only one study was included which described a collaboration within primary care as well as between primary care and secondary or tertiary care [54]. The study had grip strength as the primary outcome, which was significantly different in the intervention group at the two-month follow-up. The other health outcomes, pain and health status, showed non-significant differences at that time point.

This study also found significant results regarding quality of care experienced by patients at the two-month follow-up.

Costs and HCP work satisfaction were not measured.

\subsubsection{Between Primary Care and Social Care-Randomized Trial Design}

Bültmann et al. was the only included study evaluating collaboration between primary and social care [55]. For health outcomes, no significant changes in pain intensity and functional disability between intervention and control groups were reported at any time point.

Cumulative sickness absence hours was the primary outcome of this study. Results showed a significant decrease in sick leave at six and 12 months, compared to the control group.

Bültmann et al. did not measure outcomes on quality of care experienced by patients or HCP work satisfaction [55].

\subsubsection{Between Primary Care and Social Care-Non-Randomized Study Design}

Heijbel et al. evaluated the collaboration between primary and social care, but, in this study, health outcomes and quality of care experienced by patients were not measured [56].

For return to work, the primary outcome of this study, a significant improvement was found after two years.

Mixed results were reported regarding $\mathrm{HCP}$ work satisfaction, measuring the experiences of executing and implementing a workplace-based rehabilitation intervention.

\subsubsection{Between Primary Care and Secondary or Tertiary Care and Social Care-Randomized Trial Designs}

Two interventions in this group were evaluated, one intervention by two studies and the other by one $[57,59,60]$. Two studies measured health outcomes: pain intensity with the VAS and functional status with the Roland-Morris Disability Questionnaire $[57,60]$. Only functional status was positively changed at 12 months, whereas the other associations at 3 , 6 , and 12 months were all non-significant between the groups. 
In all three studies, the primary outcome was the duration of sick leave to a full return to work. In both studies of Lambeek, significant differences were found at the 12-month follow-up, favoring the intervention over the control group $[59,60]$. However, in the study of Anema et al., non-significant differences between interventions were found within the same timeframe [57].

No measurements were performed for the quality of care experienced by patients or $\mathrm{HCP}$ work satisfaction.

\subsubsection{Between Primary Care and Community-Based Care-Randomized Trial Designs}

Two interventions, with three randomized trial designs, of which one was a protocol, were found reporting such collaboration between primary care and community-based care. Two studies measured health outcomes $[63,66]$. McBeth et al. had change in health as the primary outcome, which was significantly more improved at six and nine months than in the control group [66]. Significant differences in changes were also found between both conditions for kinesiophobia at nine months and for the physical component of HRQoL at both time points, measured with the Short Form-36. For the mental component score, general health and chronic pain grade, no significant results were found. Bennell et al. found non-significant differences in measured health outcomes, of which knee pain intensity and physical functioning in the previous $48 \mathrm{~h}$ were the primary outcomes [63].

Costs were assessed in the study of McBeth et al. with a cost-effectiveness analysis [66]. Non-significant differences between intervention and control group at the six- and ninemonth follow-ups were found.

No measurements were performed for the quality of care experienced by patients or for HCP work satisfaction.

\subsubsection{Between Primary Care and Community-Based Care-Qualitative Designs}

Bee et al., and Hinman et al. performed qualitative evaluations for Quadruple Aim goals $[62,65]$. Mixed results were found regarding participants' illness experiences, which was a health outcome [62]. No cost outcomes were assessed qualitatively.

Quality of care experienced by patients had mixed results in the study of Bee et al. [62]. Positive as well as negative results were found regarding treatment preferences and the perceived fit with the interventions and their patients' needs. Hinman et al. measured the satisfaction of HCPs in combination with experiences of patients [65]: their only positive results regarded HCPs' interest in patients during the treatment and in collaboration. Mixed results were found regarding information and accountability, program structure, and roles and communication in teamwork.

\subsection{Risk of Bias}

The results of the risk of bias (RoB) per domain for the randomized trial designs $(n=19)$ are presented in Figure 3 and for the non-randomized study designs $(n=12)$ in Figure 4 . The results of the critical appraisal of the qualitative designs $(n=7)$ can be found in Figure 5. 


\section{Author}

1. Calner et al. (2016) [49]

2. Chemelinsky et al. (2013) [71]

4.1. Dobscha et al. (2008) [67]

4.2. Dobscha et al. (2009) [72]

5. Gustavsson et al. (2018) [69]

6. Hansson et al. (2017) [75]

7.2. Helminen et al. (2015) [73]

8.1 Nordin et al. (2016) [74]

21.2. Sundberg et al. (2009) [89]

22. Haldersen et al. (1998) [50]

23. Rothman et al. (2013) [52]
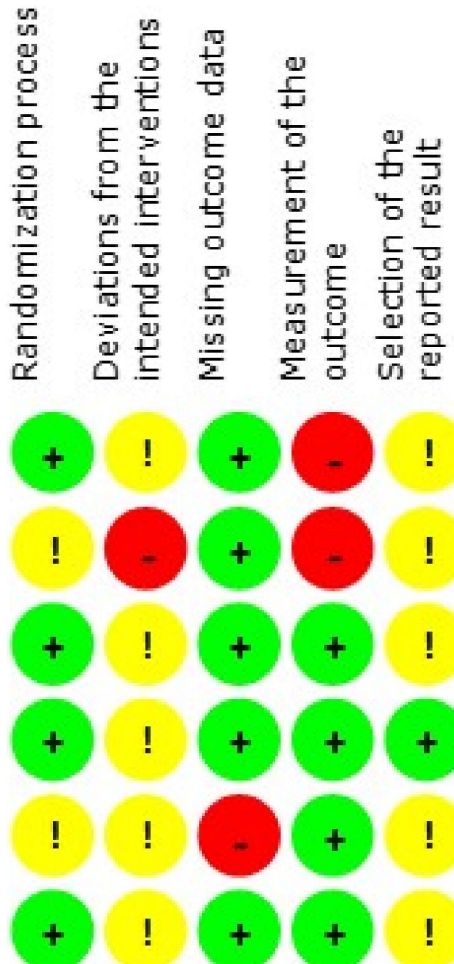

(1)
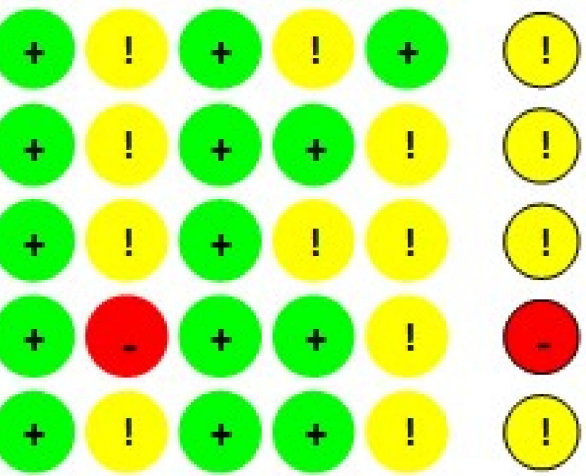

24. Taylor-Gjevre et al. (2017) [53]
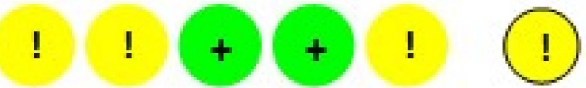

28. Stoffer-Marx et al. (2018) [54]
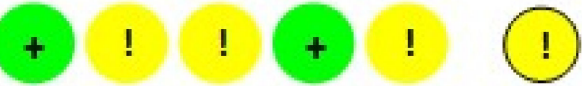

29. Bültmann et al. (2009) [55]

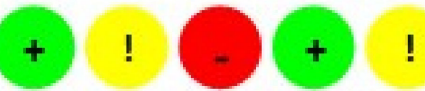

31.2. Lambeek et al. (2010) [59]
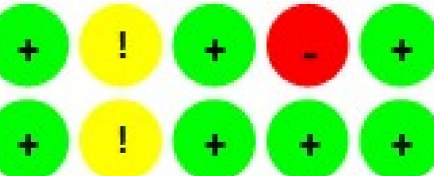

31.3 Lambeek et al. (2010) [60]
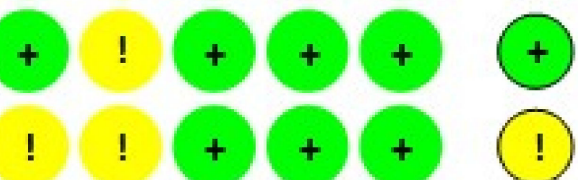

32.2 Anema et al. (2007) [57]

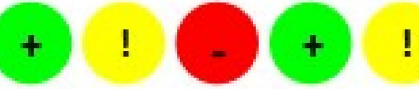

$33.1 \quad$ McBeth et al. (2012) [66]

34.3 Bennell et al. (2017) [63]

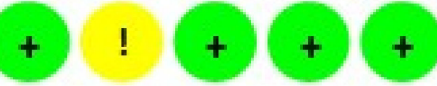

Low risk

Some concerns

High risk

Figure 3. Risk of bias of randomized trial designs. 


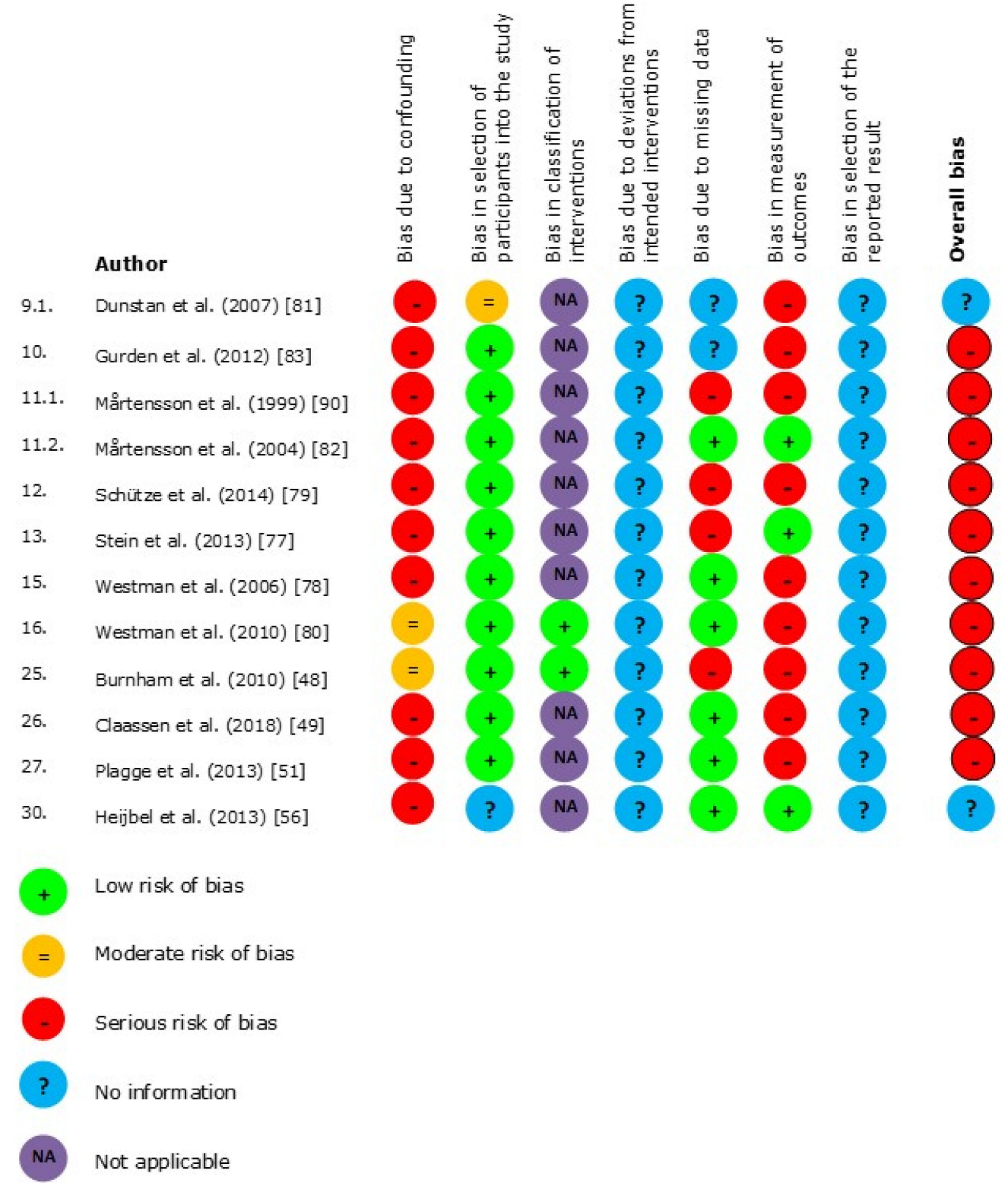

Figure 4. Risk of bias of non-randomized study designs.

\section{Author}

8.2. Nordin et al. (2017) [85]

9.2. Dunstan et al. (2014) [84]

11.3. Mårtensson et al. (2006) [87]

18.2. Lovo et al. (2019) [88]

19. Pietilä Holmner et al. (2018) [86]

33.2. Bee et al. (2016) [62]

34.2. Hinman et al. (2016) [65]

\section{Overall}

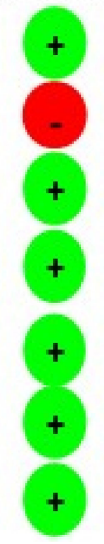

Positive methodological quality

Negative methodological quality

Figure 5. Critical appraisal qualitative designs. 


\subsection{Randomized Trial Designs}

Overall, the studies of Dobscha et al., and Lambeek et al., were found to have a low RoB $[60,72]$. Ten studies raised some concerns in the RoB, and seven studies had a high RoB. The randomization process had a low RoB for 15 studies, while four studies had some concerns $[53,57,69,71]$. Deviations from the intended interventions most often led to there being some concerns for RoB, in most cases because participants, patients and HCPs, were unblinded. Chemelinsky et al. and Halderson et al. were judged to have some concerns for this RoB domain [50,71]. Completeness of outcome data and measurements of outcomes most often had a low RoB, though there some concerns for three studies $[54,73,95]$. Six studies had a high RoB on these domains [55,60,66,69-71]. There were some concerns about possible selection of reported results with fourteen studies because most study protocols were not published, so planned outcomes could not be matched with published outcomes [49,50,52-55,66,67,69-71,74-76].

\subsection{Non-Randomized Study Designs}

The studies of Dunstan et al., and Heijbel et al. did not report enough information to assess the RoB [56,80]. All other studies were assessed as at high RoB [48-51,56,80,82-84,86,90]. The main reason was the lack of a control group in these studies, which indicated a serious RoB in the domain of confounding. The domain classification of the interventions was not applicable for studies without a control group. Moreover, this domain was found to have a serious RoB because outcome measures could be influenced by the outcome assessors and/or unblinded patients in most studies. The lack of publication of a protocol in any of these studies led to an absence of information about any bias in the selection of the reported results.

\subsection{Qualitative Designs}

Six studies scored positively in the critical appraisal of study methods $[62,65,79,85,92,93]$. The study of Dunstan et al. was described only briefly, hindering assessment, and so this study scored negatively [81]. In most studies, the philosophical perspective was not described and a statement locating the researcher culturally or theoretically was not made.

\section{Discussion}

As far as we know, this systematic review is the first to identify which interdisciplinary rehabilitation interventions have been described within primary care, and between primary care and other health care settings, delivering rehabilitation care to patients with CMP. In addition, we describe the impact of these interdisciplinary interventions in rehabilitation care for patients with CMP, in terms of the Quadruple Aim goals: health, quality of care experienced by patients, health care costs, and HCP work satisfaction. The review was based on 49 articles (34 separate interventions), including 19 randomized trials, 12 non-randomized studies, 7 qualitative studies, and 11 articles with a description of the intervention but without a description of relevant outcomes and/or results.

In summary, of the studies that examined interventions situated in primary care $(n=19)$, most did not find significantly improved health outcomes compared to care as usual. In the non-randomized designs, in general, health outcomes seemed to improve over time. However, cost outcomes and quality of care experienced by patients in intervention groups showed a mixture of significant improvements and non-improvements in both randomized and non-randomized trial designs. The differences in satisfaction levels may relate to the fact that new interventions can change the usual care pathways, which could be challenging for the patient to follow and thus lead to lower satisfaction. Alternatively, patients who were already very satisfied with the current regular health care received might experience little or even no improvements in satisfaction level after receiving the new intervention (which has also been seen in other studies on substitution of care [96-98]). However, it may be concluded that an intervention provides added value even if not all Quadruple Aim goals have shown improvement. For example, if costs decrease, patients' health and 
HCP work satisfaction increase, but the quality of care experienced by patients remains unchanged, the intervention is of added value. It is essential that all Quadruple Aim goals be assessed and that a balanced conclusion for follow-up be drawn based on these outcomes $[99,100]$. For included interventions, HCP work satisfaction was found to be improved in the intervention groups, compared with care as usual and with baseline.

For interventions between primary care and secondary or tertiary care $(n=6)$, in the randomized trial designs, no significant differences between intervention and careas-usual groups were found for most health outcomes. Over time, no improvements were seen in a restricted program containing a three-hour educational intervention [49], whereas improvements were seen in two, more extensive, interventions. These latter comprised more treatment hours, involved more health care disciplines, and consisted of an assessment and, depending on patients' needs, psychological and exercise treatments. Both of the interventions showing improvement included collaboration with a rehabilitation setting [48,51]. Unfortunately, both also displayed a serious risk of bias and, therefore, more research is warranted before drawing definite conclusions. Regarding cost outcomes, ability to work significantly improved while the return to work after 12 months did not improve in these groups $[49,50,52]$. Grant et al. found that facilitators such as managing pain, managing work, and making workplace adjustments appear to be key factors for successful return to work [101]. It could be that patients perceived their ability to work sufficiently improved for return to work, but that, for example, workplace adjustments were not yet adequate to allow this. Mixed results were reported regarding quality of care experienced by patients in two different interventions containing an interdisciplinary assessment [52,53], while this was perceived positively in a three-hour educational intervention [49].

The combined intervention in primary care and between primary care and secondary or tertiary care $(n=1)$, described by Stoffer-Marx et al., had some concerns in the risk of bias [54]. The study only showed improvements on the primary health outcome of grip strength, while no difference between groups was seen for pain and health status after two months. Patients receiving the intervention perceived the quality of care as improved after a two-month follow-up, compared to patients receiving care as usual. As this was the only intervention with an extensive interdisciplinary collaboration, no comparisons could be made. However, as future care aims to shift to clinical networks based on collaborations [30], it is important to further explore implementation in clinical practice and research with such collaborations.

An assessment and workplace intervention between primary care and social care $(n=2)$ did not show a significantly greater improvement in health outcomes for the intervention compared with care as usual [55]. Cost outcomes showed a mixture of improved and unchanged results between the intervention and care as usual at different measurement points in a randomized trial design [55], while return to work improved over time after two years in a longitudinal study [56].

In interventions between primary care and secondary or tertiary care and social care $(n=2)$, most health outcomes did not differ between patients who received a workplace intervention combined with graded activity and those who received usual care. For cost outcomes, the duration of sick leave differed between groups in two studies [59,60], but not in another [57]. This could probably be explained by the fact that, in addition to the study of Anema et al. [57], in the studies of Lambeek et al. [59,60], the intervention was extended, involving HCPs of different disciplines (such as a case manager and the patients' pre-existing specialists), potentially leading to a better effect on duration of sick leave. Due to this interdisciplinary collaboration, such interventions may have a more patient-centered focus and biopsychosocial approach, which could explain the reported results. Currently developed eHealth technologies could make it easier to work interdisciplinarily [102]. However, little research has been performed for both types of workplace interventions. This was also found in the systematic review of Skamagki et al. [103]. In contradiction to our review, they found some consistency in health outcomes for (integrated) workplace 
interventions. In contrast with our included studies, in the review of Skamagki et al., not all included studies comprised integrated, interdisciplinary interventions [103].

For interventions between primary care and community-based care $(n=2)$, most health outcomes did not differ more in the integrated care condition than with usual care $[62,64,66]$. No differences in cost outcomes were found between the groups of an intervention existing of combined cognitive behavioral therapy and prescribed exercise compared to treatment as usual (high risk of bias). Quality of care experienced by patients and HCP work satisfaction were found to have both positive and negative results in the interventions. In contrast to our results, other studies found community-based interventions to lower health care costs and improve health outcomes [80,104]. They found that patients often visit HCPs for other complaints than their actual pain or during periods of stress. Easily accessible community-based interventions could take over these kinds of health care visits to both lower costs and increase health.

\subsection{Strengths and Limitations}

This review is the first with an overview of interdisciplinary rehabilitation interventions within primary care and between primary care and other healthcare settings for patients with CMP. The interventions identified cover a broad spectrum of interdisciplinary care interventions with a wide variety of content, duration, and HCP disciplines involved. Moreover, this review is the first focusing on all Quadruple Aim outcomes for integrated interdisciplinary care interventions. Such an overview is valuable given the recommendation of the WHO that rehabilitation services should be integrated within primary care, as well as between primary, secondary, and tertiary levels of health systems, with a case-management role for primary care $[16,105]$. Another strength of our study is the classification of interventions into subgroups, facilitating comparisons of studies. This classification is based on classifications used earlier and on definitions of intervention types. Moreover, a strength of our study process was the involvement of an information specialist to ensure the quality of the search strategy. Additionally, the PRISMA guidelines for reporting reviews were used. However, a meta-analysis could not be performed as the intervention types and outcome measures were too heterogeneous.

That the selection of articles was limited to those in English, Dutch, or German may have resulted in the exclusion of valid interventions reported in other languages. Unfortunately, interventions were often not described in full detail and/or the health care settings left unclear, potentially resulting in erroneous exclusions of studies. In some studies, it was not clear in which health care setting an $\mathrm{HCP}$, for example, a physician, worked. Furthermore, it was also not always clear how a health care setting should be classified, due to differences between countries and/or the lack of appropriate descriptions. Due to time constraints, it was not possible to contact the authors for additional information. Therefore, potentially relevant interventions (reported with incomplete descriptions of content) may have been excluded in error. In this review, an interdisciplinary care network is defined based on the IASP definition [21]. Thus, all studies with a multimodal treatment provided by a multidisciplinary team (with at least one participating primary care $\mathrm{HCP}$ collaborating in assessment and/or treatment using a shared biopsychosocial model and goals) were taken into account. An alternative definition of interdisciplinary care might have led to a different selection of articles. For the randomized trial designs, positive but non-significant results (compared to the control intervention), results with no difference, and results in favor of the control intervention, were all grouped into one category (nonsignificant (-)). We chose this grouping because not all articles described the results in much detail. A more precise categorization of significant or non-significant results would have given a broader overview.

\subsection{Implications for Future Innovations and Studies}

As future health care shifts to the implementation of clinical networks, more interdisciplinary collaborations will have to be developed and evaluated in the field of rehabilitation 
for patients with CMP. As it is important that these interventions have a good fit with, and are implemented in, daily health care, we recommend applying co-creation research together with the HCP disciplines involved, patients with CMP, and other stakeholders. In the ideal situation, an evaluation of all Quadruple Aim outcomes needs to be performed with mixed methods to give a full overview of a new interdisciplinary care intervention's impact.

In order to develop, implement, and evaluate interdisciplinary care interventions across different health care settings, it is recommended that an adjusted version of the IASP definition of interdisciplinary care be used, the current one having been developed for treatments in secondary or tertiary care settings. Moreover, the Quadruple Aim was used to classify the various outcomes in four outcome domains to identify the effect of interventions in these domains. However, in our review, it was difficult to compare the effect of the various interventions on these outcomes, as a wide range of outcome measures and assessment methods were used. Due to the large variation found, a meta-analysis could not be executed. Therefore, to improve uniformity, we propose to develop a core outcome set with measurement instruments and assessment methods with standardized measurement moments for each Quadruple Aim goal (health, quality of care experienced by patients, health care costs, and HCP work satisfaction). This will facilitate future research comparing the effect of interventions. Moreover, not all study designs (e.g., mixed methods or qualitative methods) incorporated Quadruple Aim outcomes so our overview could not be complete. Therefore, it is recommended that interventions developed in the future be evaluated with mixed methods study designs. In this review, a large number of full papers had to be screened before making a decision because most abstracts, and some full papers, did not clearly describe the content of their intervention and the degree of collaboration between HCPs. We recommend that articles use reporting guidelines for abstracts and intervention details, such as the Template for Intervention Description and Replication (TIDieR) checklist [106].

\section{Conclusions}

There is a wide variety in content, collaboration, and evaluation methods of interdisciplinary rehabilitation interventions within primary care, and between primary care and other health care settings, delivering rehabilitation care for patients with CMP. Most interdisciplinary interventions are evaluated in primary care, while fewer interventions are implemented between primary care and other health care settings. It seems that interventions with the involvement of different HCP disciplines, and more patient-centered interventions, with a broader content and duration of treatment, are more effective than care as usual. Therefore, further initiatives and research have to be performed for interdisciplinary care interventions within and across health care settings for patients with CMP. These interventions have to be evaluated with mixed methods on all Quadruple Aim outcomes.

Author Contributions: C.L. was involved in data curation, formal analysis, investigation, methodology, project administration, supervision, validation, visualization, and writing-original draft. L.B. was involved in formal analysis, investigation, methodology, supervision, validation, and writingreview and editing. I.H. was involved in supervision, validation, and writing - review and editing. M.K. and J.V. were involved in supervision and writing-review and editing. All authors have read and agreed to the published version of the manuscript.

Funding: This research received no external funding.

Acknowledgments: We would like to thank Wieneke Meijer (W.M.), for her contribution to the development of the search strategy and her contribution to article selection. Furthermore, we would like to thank Gregor Franssen for his role as information specialist in this review. Scientific proofreading and English editing was done by Les Hearn (les_hearn@yahoo.co.uk).

Conflicts of Interest: The authors declare no conflict of interest. 


\section{Appendix A Literature Searches}

Search Pubmed:

(((Pain[MeSH:NoExp] OR Pain*[tiab] OR Ache*[tiab]) AND (chronic*[tiab] OR back[tiab] OR musculoskeletal ${ }^{*}\left[\right.$ tiab] OR Neck[tiab] OR cervical ${ }^{*}[$ tiab] OR lumb*[tiab] OR ankle*[tiab] OR knee $^{*}[$ tiab] OR wrist*[tiab] OR elbow*[tiab] OR shoulder*[tiab] OR hip[tiab] OR pelvic girdle[tiab]

Patient OR Physical Suffering*[tiab])) OR Arthralgia[MESH] OR arthralgia*[tiab] OR polyarthralgia*[tiab] OR Muscular Rheumatism[tiab] OR Lumbago[tiab] OR Fibromyalgi*[tiab] OR Neckache*[tiab] OR complex regional pain syndrome[tiab] OR regional pain[tiab] OR Arthritis[MeSH] OR Arthriti*[tiab] OR osteoarthr*[tiab])

AND

(((interdisciplin*[tiab] OR integrat*[tiab] OR intersect*[tiab] OR transmural[tiab] OR multidisciplinar*[tiab] OR chain*[tiab] OR Comprehensive[tiab] OR deliver*[tiab] OR network*[tiab] OR coordinat ${ }^{*}[$ tiab] OR collaboration*[tiab] OR level*[tiab] OR appropriate[tiab] OR outpatient[tiab]

OR ambulatory[tiab] OR Patient focused[tiab] OR transition*[tiab]) AND (healthcare[tiab] OR

Intervention care[tiab] OR health care[tiab] OR service*[tiab] OR system*[tiab])) OR Patient Care Management

[MeSH:NoExp] OR patient care management [tiab] OR comprehensive health care[MeSH] OR

Delivery of Health Care[MeSH] OR pain management[MeSH] OR Pain Management*[tiab] OR integrated delivery system*[tiab] OR managed clinical network*[tiab] OR Intersectoral Collaboration*[tiab] OR managed care[tiab] OR shared care[tiab])

AND

((Physical and Rehabilitation Medicine[MESH] OR physical and rehabilitation medicine[tiab] OR rehabilitation [MESH] OR rehabilitation[tiab] OR Physical Therapy Specialty[MeSH] OR physical

Comparison therap*[tiab] OR Physical Therapy Modalities[MeSH] OR Physical Therapy Modalit*[tiab] OR occupational therapy[MeSH] OR Occupational Therap*[tiab] OR Physiatry[tiab] OR Habilitation[tiab] OR physiotherap*[tiab] OR biopsychosocial[tiab] OR exercise therapy[tiab]))

AND

(Quadruple Aim[tiab] OR Triple Aim[tiab] OR health outcome*[tiab] OR quality of health care[MeSH] OR quality of healthcare[tiab] OR Population health[MeSH] OR population health[tiab]

OR Quality of Life[MeSH] OR quality of life[tiab] OR treatment Outcome[MeSH] OR treatment outcome $^{*}\left[\right.$ tiab] OR Clinical Effect ${ }^{*}[$ tiab] OR Rehabilitation Outcome*[tiab] OR Treatment

Efficacy[tiab] OR Experienced health[tiab] OR HRQOL[tiab] OR health related quality of life[tiab] OR life quality[tiab] OR patient satisfaction[MeSH] OR patient satisfaction*[tiab] OR Consumer

Outcome Satisfaction[tiab] OR patient experience*[tiab] OR meaning in work[tiab] OR meaningful work[tiab] OR workforce engagement[tiab] OR work pressure[tiab] OR job satisfaction[MeSH] OR job satisfaction*[tiab] OR work satisfaction[MeSH] OR work satisfaction*[tiab] OR workforce satisfaction[tiab] OR Health care costs[MeSH] OR Health Care Cost*[tiab] OR Health Expenditures[MeSH] OR Health Expenditure*[tiab] OR Cost-Benefit Analysis[MeSH] OR Cost-Benefit Analys*[tiab] OR Costs and Cost Analysis[MeSH] OR Costs and cost analys*[tiab] OR cost effect*[tiab] OR Costs[tiab]) 


\begin{tabular}{|c|c|}
\hline AND & \\
\hline Time & (“1994/11/1”[Date-Publication]: “3000”[Date-Publication]) \\
\hline Final & 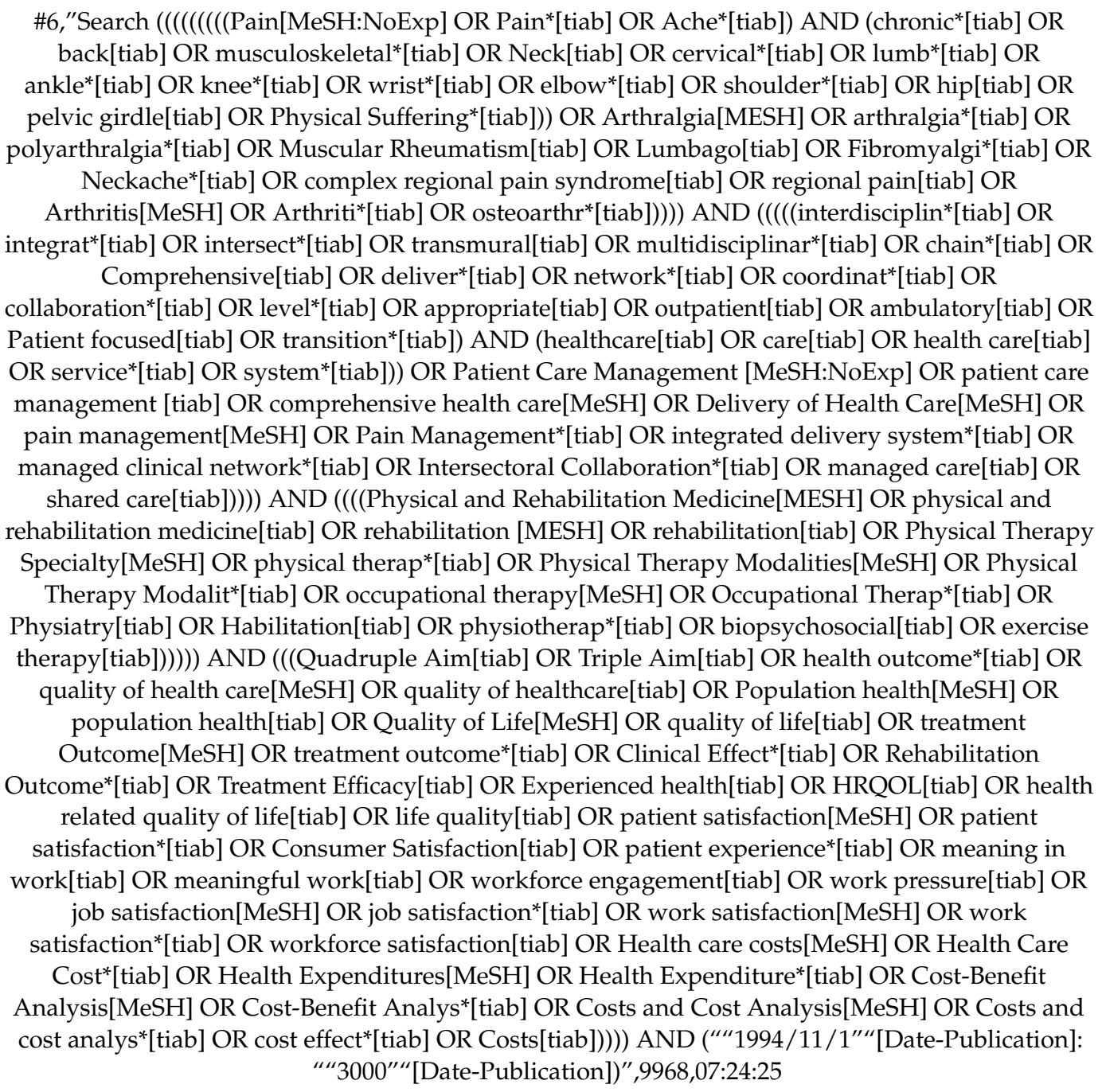 \\
\hline
\end{tabular}

Search CINAHL:

(((TI Pain OR AB Pain) OR (TI Ache OR AB ache)) AND ((TI chronic OR AB chronic) OR (TI back OR AB back) OR (TI musculoskeletal OR AB musculoskeletal) OR (TI Neck OR AB neck) OR (TI cervical OR AB cervical) OR (TI lumb OR AB lumb) OR (TI ankle OR AB ankle) OR (TI knee OR AB knee) OR (TI wrist OR AB wrist) OR (AB elbow OR TI elbow) OR (TI shoulder OR AB shoulder) OR (TI hip OR AB hip) OR (TI pelvic girdle OR AB pelvic girdle) OR (TI Physical Suffering OR AB physical

Patient suffering))) OR TI Arthralgia OR AB arthralgia OR TI polyarthralgia OR AB polyarthralgia OR TI

Muscular Rheumatism OR AB muscular rheumatism OR TI Lumbago OR AB lumbago OR TI Fibromyalgia OR AB fibromyalgia OR TI Neckache OR AB neckache OR TI complex regional pain syndrome OR AB complex regional pain syndrome OR TI regional pain OR AB regional pain OR TI Arthritis OR AB arthritis OR TI osteoarthritis OR AB osteoarthritis 


\section{AND}

((TI interdisciplinary OR AB interdisciplinary OR TI integrated OR AB integrated OR TI intersection OR AB intersection OR TI transmural OR AB transmural OR TI multidisciplinary OR AB multidisciplinary OR TI chain OR AB chain OR TI Comprehensive OR AB Comprehensive OR TI delivery OR AB delivery OR TI network OR AB network OR TI coordination OR AB coordination OR TI collaboration OR AB collaboration OR TI level OR AB level OR TI levels OR AB levels OR TI appropriate $\mathrm{OR} A B$ appropriate $\mathrm{OR}$ TI outpatient $\mathrm{OR} A B$ outpatient OR TI ambulatory OR $\mathrm{AB}$ ambulatory OR TI Patient focused OR AB patient focused OR TI transition OR AB transition) AND

Intervention

Comparison
(TI Physical and Rehabilitation Medicine AB physical and rehabilitation medicine OR TI rehabilitation OR AB rehabilitation OR TI Physical Therapy Specialty OR AB physical therapy specialty OR TI Physical Therapy Modalities OR AB Physical Therapy Modalities OR TI occupational therapy OR AB occupational therapy OR TI Physiatry OR AB physiatry OR TI Habilitation OR AB habilitation OR TI physiotherapy OR AB physiotherapy OR TI biopsychosocial OR AB biopsychosocial OR TI exercise therapy OR AB exercise therapy)

TI Quadruple Aim OR AB quadruple aim OR TI Triple Aim OR AB triple aim OR TI health outcome OR AB health outcome OR TI quality of health care OR AB quality of healthcare OR TI Population health OR AB population health OR TI Quality of Life OR AB quality of life OR TI treatment Outcome OR AB treatment outcome OR TI Clinical Effect OR AB clinical effect OR TI Rehabilitation Outcome OR AB rehabilitation outcome OR TI Treatment Efficacy OR AB treatment efficacy OR TI Experienced health OR AB experienced health OR TI HRQOL OR AB HRQOL OR TI health related quality of life OR AB health related quality of life OR TI life quality OR AB life quality OR TI patient satisfaction OR AB patient satisfaction OR TI Consumer Satisfaction OR AB consumer satisfaction

Outcome OR TI patient experience OR AB patient experience OR AB meaning in work OR TI meaning in work OR TI meaningful work OR AB meaningful work OR TI workforce engagement OR AB workforce engagement OR TI work pressure OR AB work pressure OR TI job satisfaction OR AB job satisfaction OR TI work satisfaction OR AB work satisfaction OR TI workforce satisfaction OR AB workforce satisfaction OR TI Health care costs OR AB Health Care Costs OR TI Health Expenditures OR AB Health Expenditures OR TI Cost-Benefit Analysis OR AB Cost-Benefit Analysis OR TI Costs and Cost Analysis OR AB Costs and cost analysis OR TI cost effectivity OR AB cost effectivity OR TI Costs OR 
AND

Time

DT 1994-2019

(((TI Pain OR AB Pain) OR (TI Ache OR AB ache)) AND ((TI chronic OR AB chronic) OR (TI back OR AB back) OR (TI musculoskeletal OR AB musculoskeletal) OR (TI Neck OR AB neck) OR (TI cervical OR AB cervical) OR (TI lumb OR AB lumb) OR (TI ankle OR AB ankle) OR (TI knee OR AB knee) OR (TI wrist OR AB wrist) OR (AB elbow OR TI elbow) OR (TI shoulder OR AB shoulder) OR (TI hip OR AB hip) OR (TI pelvic girdle OR AB pelvic girdle) OR (TI Physical Suffering OR AB physical suffering))) OR TI Arthralgia OR AB arthralgia OR TI polyarthralgia OR AB polyarthralgia OR TI Muscular Rheumatism OR AB muscular rheumatism OR TI Lumbago OR AB lumbago OR TI Fibromyalgia OR AB fibromyalgia OR TI Neckache OR AB neckache OR TI complex regional pain syndrome OR AB complex regional pain syndrome OR TI regional pain OR AB regional pain OR TI Arthritis OR AB arthritis OR TI osteoarthritis OR AB osteoarthritis) AND (((TI interdisciplinary OR $A B$ interdisciplinary OR TI integrated OR AB integrated OR TI intersection OR AB intersection OR TI transmural OR AB transmural OR TI multidisciplinary OR AB multidisciplinary OR TI chain OR AB chain OR TI Comprehensive OR AB Comprehensive OR TI delivery OR AB delivery OR TI network OR AB network OR TI coordination OR AB coordination OR TI collaboration OR AB collaboration OR TI level OR AB level OR TI levels OR AB levels OR TI appropriate OR AB appropriate OR TI outpatient OR AB outpatient OR TI ambulatory OR AB ambulatory OR TI Patient focused OR AB patient focused OR TI transition OR AB transition) AND (TI healthcare OR AB healthcare OR TI care OR AB care OR TI health care OR AB health care OR TI service OR AB service OR TI system OR AB

system)) OR TI Patient Care Management OR AB patient care management OR TI patient care

management OR AB patient care management OR TI comprehensive health care OR AB comprehensive health care OR TI Delivery of Health Care OR AB delivery of health care OR TI pain management OR AB pain management OR TI integrated delivery system OR AB integrated delivery

system OR TI managed clinical network OR AB managed clinical network OR TI Intersectoral Collaboration OR AB intersectoral collaboration OR TI managed care OR AB managed care OR TI shared care OR AB shared care) AND ((TI Physical and Rehabilitation Medicine AB physical and rehabilitation medicine OR TI rehabilitation OR AB rehabilitation OR TI Physical Therapy Specialty

OR AB physical therapy specialty OR TI Physical Therapy Modalities OR AB Physical Therapy

Modalities OR TI occupational therapy OR AB occupational therapy OR TI Physiatry OR AB physiatry OR TI Habilitation OR AB habilitation OR TI physiotherapy OR AB physiotherapy OR TI biopsychosocial OR AB biopsychosocial OR TI exercise therapy OR AB exercise therapy)) AND (TI Quadruple Aim OR AB quadruple aim OR TI Triple Aim OR AB triple aim OR TI health outcome OR $A B$ health outcome OR TI quality of health care OR AB quality of healthcare OR TI Population health OR AB population health OR TI Quality of Life OR AB quality of life OR TI treatment Outcome OR AB treatment outcome OR TI Clinical Effect OR AB clinical effect OR TI Rehabilitation Outcome OR AB rehabilitation outcome OR TI Treatment Efficacy OR AB treatment efficacy OR TI Experienced health OR AB experienced health OR TI HRQOL OR AB HRQOL OR TI health related quality of life OR AB health related quality of life OR TI life quality OR AB life quality OR TI patient satisfaction OR AB patient satisfaction OR TI Consumer Satisfaction OR AB consumer satisfaction OR TI patient experience $\mathrm{OR} A B$ patient experience OR AB meaning in work OR TI meaning in work OR TI meaningful work OR AB meaningful work OR TI workforce engagement OR AB workforce engagement OR TI work pressure OR AB work pressure OR TI job satisfaction OR AB job satisfaction OR TI work satisfaction OR AB work satisfaction OR TI workforce satisfaction OR AB workforce satisfaction OR TI Health care costs OR AB Health Care Costs OR TI Health Expenditures OR AB Health Expenditures OR TI Cost-Benefit Analysis OR AB Cost-Benefit Analysis OR TI Costs and Cost Analysis OR AB Costs and cost analysis OR TI cost effectivity OR AB cost effectivity OR TI Costs OR $\mathrm{AB}$ costs)

Limiters-Published Date: 19941101-20191131

Expanders-Apply related words; Apply equivalent subjects Search modes-Boolean/Phrase

Search web of science:

(((Pain* OR Ache*) AND (chronic ${ }^{*}$ OR musculoskeletal* OR Neck OR cervical* OR lumb* OR ankle*

Patient OR knee* OR wrist* OR elbow* OR shoulder* OR hip* OR pelvic girdle OR Physical Suffering*)) OR arthralgia* OR polyarthralgia* OR "Muscular Rheumatism*" OR Lumbago OR Fibromyalgi* OR Neckache* OR "complex regional pain syndrome" OR "regional pain" OR Arthriti* OR osteoarthritis) 
AND

(((interdisciplinary OR integrated OR intersectoral OR transmural OR multidisciplinary OR chain*

OR Comprehensive OR deliver* OR network* OR coordination OR collaboration* OR level* OR care OR health care OR service* OR system*)) OR "Patient Care Management" OR "comprehensive health care" OR "Delivery of Health Care" OR "pain management" OR "integrated delivery system*" OR "managed clinical network" OR "Intersectoral Collaboration*" OR "managed care" OR "shared care")

AND

Comparison

("Physical and Rehabilitation Medicine" OR rehabilitation OR "Physical Therapy Specialty" OR "physical therap*" OR "Physical Therapy Modalit*" OR "occupational therap*" OR Physiatr* OR Habilitation OR physiotherap* OR biopsychosocial treatment OR "exercise therapy")

AND

(“Quadruple Aim" OR “Triple Aim" OR health outcome* OR "quality of health care" OR "quality of healthcare" OR "Population health" OR “Quality of Life" OR "treatment Outcome" OR "Clinical

Effect*" OR "Rehabilitation Outcome*" OR “Treatment Efficacy" OR “Experienced health" OR HRQOL OR "health related quality of life" OR "life quality" OR "patient satisfaction" OR

Outcome

"Consumer Satisfaction" OR "patient experience

OR "workforce engagement" OR "work pressure" OR "job satisfaction" OR "work satisfaction" OR

"workforce satisfaction" OR "Health care cost"” OR "Health Expenditure*" OR "Cost-Benefit Analys" OR "Costs and Cost Analys" OR "cost effect" OR Costs)

AND

Time 1994-2019

TOPIC: ((((Pain* OR Ache*) AND (chronic* OR musculoskeletal* OR Neck OR cervical* OR lumb* OR ankle* OR knee* OR wrist* OR elbow* OR shoulder* OR hip* OR pelvic girdle OR Physical Suffering*)) OR arthralgia* OR polyarthralgia* OR "Muscular Rheumatism*" OR Lumbago OR Fibromyalgi* OR Neckache* OR “complex regional pain syndrome" OR "regional pain" OR Arthriti* OR osteoarthritis)) AND TOPIC: ((((interdisciplinary OR integrated OR intersectoral OR transmural OR multidisciplinary OR chain* OR Comprehensive OR deliver* OR network* OR coordination OR collaboration* OR level* OR appropriate OR outpatient OR ambulatory OR Patient focused OR transitional) AND (healthcare OR care OR health care OR service* OR system*)) OR "Patient Care

Management" OR "comprehensive health care" OR "Delivery of Health Care" OR "pain management" OR "integrated delivery system*" OR "managed clinical network*" OR "Intersectoral Collaboration*" OR "managed care" OR "shared care")) AND TOPIC: (("Physical and Rehabilitation Medicine" OR rehabilitation OR "Physical Therapy Specialty" OR "physical therap" ${ }^{*}$ OR "Physical Therapy Modalit*" OR "occupational therap*" OR Physiatr* OR Habilitation OR physiotherap* OR biopsychosocial treatment OR “exercise therapy")) AND TOPIC: ((“Quadruple Aim" OR “Triple Aim" OR health outcome* OR "quality of health care" OR "quality of healthcare" OR "Population health" OR "Quality of Life" OR "treatment Outcome" OR "Clinical Effect" OR "Rehabilitation

Outcome*" OR "Treatment Efficacy" OR "Experienced health" OR HRQOL OR "health related quality of life" OR "life quality" OR "patient satisfaction" OR "Consumer Satisfaction" OR "patient experience*" OR "meaning in work" OR "meaningful work" OR "workforce engagement" OR "work pressure" OR "job satisfaction" OR "work satisfaction" OR "workforce satisfaction" OR "Health care cost" OR "Health Expenditure*" OR "Cost-Benefit Analys" OR "Costs and Cost Analys" OR "cost effect*" OR Costs))

Timespan: 1994-2019. Indexes: SCI-EXPANDED, SSCI, A\&HCI, CPCI-S, CPCI-SSH, ESCI.

Search in psycinfo:

(((Pain* OR Ache*) AND (chronic* OR musculoskeletal* OR Neck OR cervical* OR lumb* OR ankle*

Patient OR knee* OR wrist* OR elbow* OR shoulder* OR hip* OR pelvic girdle OR Physical Suffering*)) OR arthralgia* OR polyarthralgia* OR "Muscular Rheumatism*" OR Lumbago OR Fibromyalgi* OR Neckache* OR "complex regional pain syndrome" OR "regional pain" OR Arthriti* OR osteoarthritis) 
AND

(((interdisciplinary OR integrated OR intersectoral OR transmural OR multidisciplinary OR chain*

OR Comprehensive OR deliver* OR network* OR coordination OR collaboration* OR level* OR

Intervention appropriate OR outpatient OR ambulatory OR Patient focused OR transitional) AND (healthcare OR care OR health care OR service* OR system*)) OR "Patient Care Management" OR "comprehensive health care" OR "Delivery of Health Care" OR "pain management" OR "integrated delivery system*" OR "managed clinical network" OR "Intersectoral Collaboration*" OR "managed care" OR "shared care")

\begin{tabular}{|c|c|}
\hline AND & \\
\hline Comparison & $\begin{array}{c}\text { ("Physical and Rehabilitation Medicine" OR rehabilitation OR "Physical Therapy Specialty" OR } \\
\text { "physical therap*" OR "Physical Therapy Modalit*" OR “occupational therap*" OR Physiatr* OR } \\
\text { Habilitation OR physiotherap* OR biopsychosocial OR “exercise therapy") }\end{array}$ \\
\hline \multicolumn{2}{|l|}{ AND } \\
\hline Outcome & 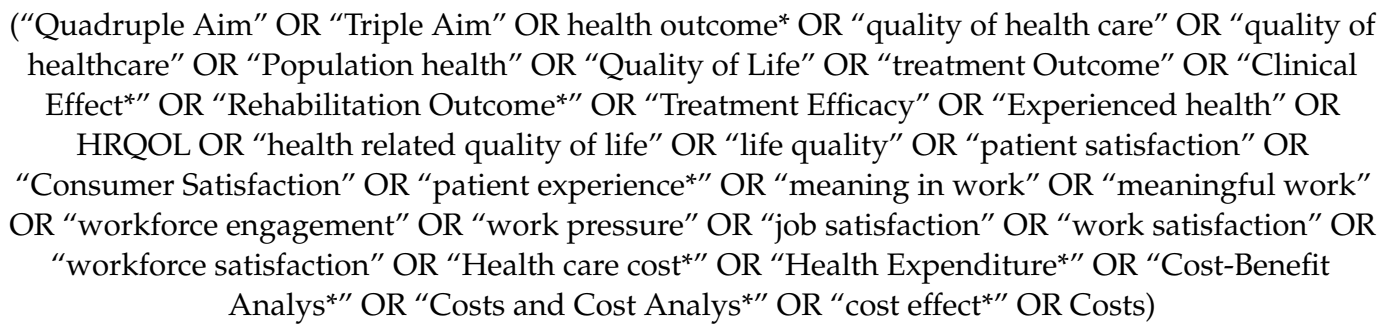 \\
\hline
\end{tabular}

AND

Time 1994-2019

((Pain* OR Ache*) AND (chronic* OR musculoskeletal* OR Neck OR cervical* OR lumb* OR ankle* OR knee* OR wrist* OR elbow* OR shoulder* OR hip* OR pelvic girdle OR Physical Suffering*)) OR arthralgia* OR polyarthralgia* OR “Muscular Rheumatism*” OR Lumbago OR Fibromyalgi* OR

Neckache* OR "complex regional pain syndrome" OR "regional pain" OR Arthriti* OR osteoarthritis)) AND (((interdisciplinary OR integrated OR intersectoral OR transmural OR multidisciplinary OR chain* OR Comprehensive OR deliver* OR network* OR coordination OR collaboration* OR level* OR appropriate OR outpatient OR ambulatory OR Patient focused OR transitional) AND (healthcare OR care OR health care OR service* OR system*)) OR "Patient Care

Management" OR "comprehensive health care" OR "Delivery of Health Care" OR "pain management" OR "integrated delivery system*" OR "managed clinical network*" OR "Intersectoral

Collaboration*" OR "managed care" OR "shared care")) AND (("Physical and Rehabilitation Medicine" OR rehabilitation OR "Physical Therapy Specialty" OR "physical therap" ${ }^{*}$ OR "Physical Therapy Modalit*" OR "occupational therap*" OR Physiatr* OR Habilitation OR physiotherap* OR biopsychosocial OR “exercise therapy”)) AND ((“Quadruple Aim” OR “Triple Aim” OR health outcome* OR "quality of health care" OR "quality of healthcare" OR "Population health" OR "Quality of Life" OR "treatment Outcome" OR "Clinical Effect*" OR "Rehabilitation Outcome*" OR "Treatment Efficacy" OR "Experienced health" OR HRQOL OR "health related quality of life" OR "life quality" OR "patient satisfaction" OR "Consumer Satisfaction" OR "patient experience*" OR "meaning in work" OR "meaningful work" OR "workforce engagement" OR "work pressure" OR "job satisfaction" OR "work satisfaction" OR "workforce satisfaction" OR "Health care cost*" OR "Health Expenditure" ${ }^{* \prime}$ OR “Cost-Benefit Analys" ${ }^{* \prime}$ OR "Costs and Cost Analys" OR "cost effect*" OR Costs))

Limiters-Published Date: 19941101-20191131

Expanders-Apply related words; Apply equivalent subjects Search modes-Boolean/Phrase

\section{References}

1. Breivik, H.; Collett, B.; Ventafridda, V.; Cohen, R.; Gallacher, D. Survey of chronic pain in Europe: Prevalence, impact on daily life, and treatment. Eur. J. Pain 2006, 10, 287. [CrossRef] [PubMed]

2. Cieza, A.; Causey, K.; Kamenov, K.; Hanson, S.W.; Chatterji, S.; Vos, T. Global estimates of the need for rehabilitation based on the Global Burden of Disease study 2019: A systematic analysis for the Global Burden of Disease Study. Lancet 2020, 396, 2006-2017. [CrossRef] 
3. Jordan, K.P.; Jöud, A.; Bergknut, C.; Croft, P.; Edwards, J.J.; Peat, G.; Petersson, I.F.; Turkiewicz, A.; Wilkie, R.; Englund, M. International comparisons of the consultation prevalence of musculoskeletal conditions using population-based healthcare data from England and Sweden. Ann. Rheum. Dis. 2013, 73, 212-218. [CrossRef] [PubMed]

4. Gaskin, D.J.; Richard, P. The economic costs of pain in the United States. J. Pain 2012, 13, 715-724. [CrossRef]

5. Breivik, H.; Eisenberg, E.; O'Brien, T. The individual and societal burden of chronic pain in Europe: The case for strategic prioritisation and action to improve knowledge and availability of appropriate care. BMC Public Health 2013, 13, 1229. [CrossRef]

6. $\quad$ Bekkering, G.E.; Bala, M.M.; Reid, K.; Kellen, E.; Harker, J.; Riemsma, R.; Huygen, F.J.P.M.; Kleijnen, J. Epidemiology of chronic pain and its treatment in The Netherlands. Neth. J. Med. 2011, 69, 141-153.

7. Hoy, D.G.; Smith, E.; Cross, M.; Sanchez-Riera, L.; Buchbinder, R.; Blyth, F.M.; Brooks, P.; Woolf, A.D.; Osborne, R.H.; Fransen, M.; et al. The global burden of musculoskeletal conditions for 2010: An overview of methods. Ann. Rheum. Dis. 2014, 73, 982-989. [CrossRef]

8. Brooks, P.M. The burden of musculoskeletal disease-A global perspective. Clin. Rheumatol. 2006, 25, 778-781. [CrossRef]

9. Coleman, K.; Mattke, S.; Perrault, P.J.; Wagner, E.H. Untangling practice redesign from disease management: How do we best care for the chronically ill? Annu. Rev. Public Health 2009, 30, 385-408. [CrossRef]

10. Anderson, G.; Knickman, J.R. Changing the chronic care system to meet people's needs. Health Aff. 2001, 20, 146-160. [CrossRef]

11. Wagner, E.H.; Austin, B.T.; Davis, C.; Hindmarsh, M.; Schaefer, J.; Bonomi, A. Improving chronic illness care: Translating evidence into action. Health Aff. 2001, 20, 64-78. [CrossRef]

12. Smith, B.H.; Torrance, N. Management of chronic pain in primary care. Curr. Opin. Support. Palliat. Care 2011, 5, 137-142. [CrossRef]

13. Starfield, B.; Shi, L.; Macinko, J. Contribution of primary care to health systems and health. Milbank Q. 2005, 83, 457-502. [CrossRef]

14. Rothman, A.A.; Wagner, E.H. Chronic illness management: What is the role of primary care? Ann. Intern. Med. 2003, 138, 256-261. [CrossRef]

15. Boeckxstaens, P.; Maeseneer, J.; Sutter, A. The role of general practitioners and family physicians in the management of multimorbidity. In Comorbidity of Mental and Physical Disorders; Karger Publishers: Basel, Switzerland, 2015 ; pp. 129-147.

16. World Health Organization. Rehabilitation in Health Systems; WHO: Geneva, Switzerland, 2017.

17. Gatchel, R.J.; Peng, Y.B.; Peters, M.L.; Fuchs, P.N.; Turk, D.C. The biopsychosocial approach to chronic pain: Scientific advances and future directions. Psychol. Bull. 2007, 133, 581-624. [CrossRef]

18. Kamper, S.J.; Apeldoorn, A.T.; Chiarotto, A.; Smeets, R.J.; Ostelo, R.W.J.G.; Guzman, J.; Van Tulder, M.W. Multidisciplinary biopsychosocial rehabilitation for chronic low back pain. Cochrane Database Syst. Rev. 2014, 2014, CD000963. [CrossRef]

19. Gatchel, R.J.; McGeary, D.D.; Peterson, A.; Moore, M.; LeRoy, K.; Isler, W.C.; Hryshko-Mullen, A.S.; Edell, T. Preliminary findings of a randomized controlled trial of an interdisciplinary military pain program. Mil. Med. 2009, 174, 270-277. [CrossRef]

20. Stanos, S.; Houle, T.T. Multidisciplinary and interdisciplinary management of chronic pain. Phys. Med. Rehabil. Clin. N. Am. 2006, 17, 435-450. [CrossRef]

21. International Association for the Study of Pain Terminology Working Group. Classification of Chronic Pain, 2nd ed.; IASP: Washington, DC, USA, 2011; Available online: https:/ / www.iasp-pain.org/PublicationsNews/Content.aspx?ItemNumber=1673 \&.navItemNumber $=677$ (accessed on 6 May 2021).

22. Berwick, D.M.; Nolan, T.W.; Whittington, J. The triple aim: Care, health, and cost. Health Aff. 2008, 27, 759-769. [CrossRef]

23. Bodenheimer, T.; Sinsky, C. From triple to quadruple aim: Care of the patient requires care of the provider. Ann. Fam. Med. 2014, 12, 573-576. [CrossRef]

24. D'Alleva, A.; Leigheb, F.; Rinaldi, C.; Di Stanislao, F.; Vanhaecht, K.; De Ridder, D.; Bruyneel, L.; Cangelosi, G.; Panella, M. Achieving quadruple aim goals through clinical networks: A systematic review. J. Health Qual. Res. 2019, 34, 29-39. [CrossRef] [PubMed]

25. Purcell, N.; Zamora, K.; Tighe, J.; Li, Y.; Douraghi, M.; Seal, K. The integrated pain team: A mixed-methods evaluation of the impact of an embedded interdisciplinary pain care intervention on primary care team satisfaction, confidence, and perceptions of care effectiveness. Pain Med. 2017, 19, 1748-1763. [CrossRef] [PubMed]

26. Metzelthin, S.F.; Daniëls, R.; Van Rossum, E.; Cox, K.; Habets, H.; De Witte, L.P.; Kempen, G.I. A nurse-led interdisciplinary primary care approach to prevent disability among community-dwelling frail older people: A large-scale process evaluation. Int. J. Nurs. Stud. 2013, 50, 1184-1196. [CrossRef] [PubMed]

27. Moher, D.; Liberati, A.; Tetzlaff, J.; Altman, D.G.; The PRISMA Group. Preferred reporting items for systematic reviews and meta-analyses: The PRISMA statement. PLoS Med. 2009, 6. [CrossRef] [PubMed]

28. Liberati, A.; Altman, D.G.; Tetzlaff, J.; Mulrow, C.D.; Gøtzsche, P.C.; Ioannidis, J.P.; Clarke, M.F.; Devereaux, P.J.; Kleijnen, J.; Moher, D. The PRISMA statement for reporting systematic reviews and meta-analyses of studies that evaluate health care interventions: Explanation and elaboration. Ann. Intern. Med. 2009, 151. [CrossRef] [PubMed]

29. Ouzzani, M.; Hammady, H.; Fedorowicz, Z.; Elmagarmid, A. Rayyan-A web and mobile app for systematic reviews. Syst. Rev. 2016, 5, 1-10. [CrossRef] [PubMed]

30. World Health Organization. Rehabilitation. 2020. Available online: https://www.who.int/news-room/fact-sheets/detail/ rehabilitation (accessed on 23 December 2020). 
31. Wright, J.; Williams, R.; Wilkinson, J.R. Health needs assessment: Development and importance of health needs assessment. Br. Med. J. 1998, 316, 1310-1313. [CrossRef] [PubMed]

32. Ainpradub, K.; Sitthipornvorakul, E.; Janwantanakul, P.; van der Beek, A.J. Effect of education on non-specific neck and low back pain: A meta-analysis of randomized controlled trials. Man. Ther. 2016, 22, 31-41. [CrossRef]

33. Egmond, D.L.; Schuitemaker, R.; Mink, A.J.F. Extremiteiten: Manuele Therapie in Enge en Ruime Zin; Bohn Stafleu van Loghum: Houten, The Netherlands, 2014.

34. McNeely, M.L.; Campbell, K.; Ospina, M.; Rowe, B.H.; Dabbs, K.; Klassen, T.P.; Mackey, J.; Courneya, K. Exercise interventions for upper-limb dysfunction due to breast cancer treatment. Cochrane Database Syst. Rev. 2010, 2010, CD005211. [CrossRef]

35. Mishra, S.I.; Scherer, R.W.; Geigle, P.M.; Berlanstein, D.R.; Topaloglu, O.; Gotay, C.C.; Snyder, C. Exercise interventions on health-related quality of life for cancer survivors. Cochrane Database Syst. Rev. 2012, 2012, CD007566. [CrossRef]

36. Husebø, A.M.L.; Husebø, T.L. Quality of life and breast cancer: How can mind-body exercise therapies help? An overview study. Sports 2017, 5, 79. [CrossRef]

37. Lindström, I.; Öhlund, C.; Eek, C.; Wallin, L.; Peterson, L.-E.; Fordyce, W.E.; Nachemson, A.L. The effect of graded activity on patients with subacute low back pain: A randomized prospective clinical study with an operant-conditioning behavioral approach. Phys. Ther. 1992, 72, 279-290. [CrossRef]

38. Linton, S.J.; Hellsing, A.-L.; Andersson, D. A controlled study of the effects of an early intervention on acute musculoskeletal pain problems. Pain 1993, 54, 353-359. [CrossRef]

39. Ostelo, R.W.J.G.; De Vet, H.C.W.; Berfelo, M.W.; Kerckhoffs, M.R.; Vlaeyen, J.W.S.; Wolters, P.M.J.C.; Brandt, P.A.V.D. Effectiveness of behavioral graded activity after first-time lumbar disc surgery: Short term results of a randomized controlled trial. Eur. Spine J. 2003, 12, 637-644. [CrossRef]

40. Fordyce, W.E.; Fowler, R.S.; Lehmann, J.F.; DeLateur, B.J.; Sand, P.L.; Trieschmann, R.B. Operant conditioning in the treatment of chronic pain. Arch. Phys. Med. Rehabil. 1973, 54, 399-408.

41. Vlaeyen, J.W.S.; Haazen, I.W.C.J.; Schuerman, J.A.; Kole-Snijders, A.M.J.; Eek, H. Behavioural rehabilitation of chronic low back pain: Comparison of an operant treatment, an operant-cognitive treatment and an operant-respondent treatment. Br. J. Clin. Psychol. 1995, 34, 95-118. [CrossRef]

42. Centers for Disease Control and Prevention Division of Population Health National Center for Chronic Disease Prevention and Health Promotion. Workplace Health Model. 2016. Available online: https://www.cdc.gov/workplacehealthpromotion/model/ index.html (accessed on 6 May 2021).

43. Egli, S.; Pfister, M.; Ludin, S.M.; De La Vega, K.P.; Busato, A.; Fischer, L. Long-term results of therapeutic local anesthesia (neural therapy) in 280 referred refractory chronic pain patients. BMC Complement. Altern. Med. 2015, 15, 200. [CrossRef]

44. Pellegrin, K.; Chan, F.; Pagoria, N.; Jolson-Oakes, S.; Uyeno, R.; Levin, A. A statewide medication management system: Health information exchange to support drug therapy optimization by pharmacists across the continuum of care. Appl. Clin. Inform. 2018, 9, 1-10. [CrossRef] [PubMed]

45. Higgins, J.P.T.; Altman, D.G.; Gøtzsche, P.C.; Jüni, P.; Moher, D.; Oxman, A.D.; Savović, J.; Schulz, K.F.; Weeks, L.; Sterne, J.A.C.; et al. The Cochrane Collaboration's tool for assessing risk of bias in randomised trials. Br. Med. J. 2011, 343 , d5928. [CrossRef] [PubMed]

46. Sterne, J.A.; Hernán, M.A.; Reeves, B.C.; Savović, J.; Berkman, N.D.; Viswanathan, M.; Henry, D.; Altman, D.G.; Ansari, M.T.; Boutron, I.; et al. ROBINS-I: A tool for assessing risk of bias in non-randomised studies of interventions. Br. Med. J. 2016, 355, i4919. [CrossRef] [PubMed]

47. The Joanna Briggs Institute. Critical Appraisal Tools, Checklist for Qualitative Research; JBI: Adelaide, Australia, 2020.

48. Burnham, R.; Day, J.; Dudley, W. Multidisciplinary chronic pain management in a rural Canadian setting. Can. J. Rural Med. 2010, 15, 7-13.

49. Claassen, A.A.O.M.; Schers, H.J.; Koëter, S.; Van Der Laan, W.H.; Kremers-van de Hei, K.C.A.L.C.; Botman, J.; Busch, V.J.J.F.; Rijnen, W.H.C.; van den Ende, C.H.M. Preliminary effects of a regional approached multidisciplinary educational program on healthcare utilization in patients with hip or knee osteoarthritis: An observational study. BMC Fam. Pract. 2018, 19, 82. [CrossRef]

50. Haldorsen, E.M.; Kronholm, K.; Skouen, J.S.; Ursin, H. Multimodal cognitive behavioral treatment of patients sicklisted for musculoskeletal pain: A randomized controlled study. Scand. J. Rheumatol. 1998, 27, 16-25.

51. Plagge, J.M.; Lu, M.W.; Lovejoy, T.I.; Karl, A.I.; Dobscha, S.K. Treatment of comorbid pain and PTSD in returning veterans: A collaborative approach utilizing behavioral activation. Pain Med. 2013, 14, 1164-1172. [CrossRef]

52. Rothman, M.G.; Ortendahl, M.; Rosenblad, A.; Johansson, A.C. Improved quality of life, working ability, and patient satisfaction after a pretreatment multimodal assessment method in patients with mixed chronic muscular pain: A randomized-controlled study. Clin. J. Pain 2013, 29, 195-204. [CrossRef]

53. Taylor-Gjevre, R.; Nair, B.; Bath, B.; Okpalauwaekwe, U.; Sharma, M.; Penz, E.; Trask, C.; Stewart, S.A. Addressing rural and remote access disparities for patients with inflammatory arthritis through video-conferencing and innovative inter-professional care models. Musculoskelet. Care 2018, 16, 90-95. [CrossRef]

54. Stoffer-Marx, M.A.; Klinger, M.; Luschin, S.; Meriaux-Kratochvila, S.; Zettel-Tomenendal, M.; Nell-Duxneuner, V.; Zwerina, J.; Kjeken, I.; Hackl, M.; Öhlinger, S.; et al. Functional consultation and exercises improve grip strength in osteoarthritis of the hand-A randomised controlled trial. Arthritis Res. 2018, 20, 253. [CrossRef] 
55. Bültmann, U.; Sherson, D.; Olsen, J.; Hansen, C.L.; Lund, T.; Kilsgaard, J. Coordinated and tailored work rehabilitation: A randomized controlled trial with economic evaluation undertaken with workers on sick leave due to musculoskeletal disorders. J. Occup. Rehabil. 2009, 19, 81-93. [CrossRef]

56. Heijbel, B.; Josephson, M.; Vingård, E. Implementation of a rehabilitation model for employees on long-term sick leave in the public sector: Difficulties, counter-measures, and outcomes. Work 2013, 45, 323-333. [CrossRef]

57. Anema, J.R.; Steenstra, I.A.; Bongers, P.M.; de Vet, H.C.; Knol, D.L.; Loisel, P.; van Mechelen, W. Multidisciplinary rehabilitation for subacute low back pain: Graded activity or workplace intervention or both? A randomized controlled trial. Spine 2007, 32, 291-298. [CrossRef]

58. Lambeek, L.C.; Anema, J.R.; Van Royen, B.J.; Buijs, P.C.; Wuisman, P.I.; Van Tulder, M.W.; Van Mechelen, W. Multidisciplinary outpatient care program for patients with chronic low back pain: Design of a randomized controlled trial and cost-effectiveness study [ISRCTN28478651]. BMC Public Health 2007, 7, 254. [CrossRef] [PubMed]

59. Lambeek, L.C.; Bosmans, J.E.; Van Royen, B.J.; Van Tulder, M.W.; Van Mechelen, W.; Anema, J.R. Effect of integrated care for sick listed patients with chronic low back pain: Economic evaluation alongside a randomised controlled trial. Br. Med. J. 2010, 341, c6414. [CrossRef]

60. Lambeek, L.C.; Van Mechelen, W.; Knol, D.L.; Loisel, P.; Anema, J.R. Randomised controlled trial of integrated care to reduce disability from chronic low back pain in working and private life. Br. Med. J. 2010, 340, c1035. [CrossRef]

61. Steenstra, I.A.; Anema, J.R.; Bongers, P.M.; De Vet, H.C.W.; Van Mechelen, W. Cost effectiveness of a multi-stage return to work program for workers on sick leave due to low back pain, design of a population based controlled trial [ISRCTN60233560]. BMC Musculoskelet. Disord. 2003, 4, 26. [CrossRef]

62. Bee, P.; McBeth, J.; Macfarlane, G.J.; Lovell, K. Managing chronic widespread pain in primary care: A qualitative study of patient perspectives and implications for treatment delivery. BMC Musculoskelet. Disord. 2016, 17, 354. [CrossRef]

63. Bennell, K.L.; Campbell, P.K.; Egerton, T.; Metcalf, B.; Kasza, J.; Forbes, A.; Bills, C.; Gale, J.; Harris, A.; Kolt, G.S.; et al. Telephone coaching to enhance a home-based physical activity program for knee osteoarthritis: A randomized clinical trial. Arthritis Care Res. 2017, 69, 84-94. [CrossRef]

64. Bennell, K.L.; Egerton, T.; Bills, C.; Gale, J.; Kolt, G.S.; Bunker, S.J.; Hunter, D.J.; Brand, C.A.; Forbes, A.; Harris, A.; et al. Addition of telephone coaching to a physiotherapist-delivered physical activity program in people with knee osteoarthritis: A randomised controlled trial protocol. BMC Musculoskelet. Disord. 2012, 13, 246. [CrossRef]

65. Hinman, R.S.; Delany, C.M.; Campbell, P.K.; Gale, J.; Bennell, K.L. Physical therapists, telephone coaches, and patients with knee osteoarthritis: Qualitative study about working together to promote exercise adherence. Phys. Ther. 2016, 96, 479-493. [CrossRef] [PubMed]

66. McBeth, J.; Prescott, G.; Scotland, G.; Lovell, K.; Keeley, P.; Hannaford, P.; McNamee, P.; Symmons, D.P.M.; Woby, S.; Gkazinou, C.; et al. Cognitive behavior therapy, exercise, or both for treating chronic widespread pain. Arch. Intern. Med. 2012, 172, 48-57. [CrossRef]

67. Dobscha, S.K.; Corson, K.; Leibowitz, R.Q.; Sullivan, M.D.; Gerrity, M.S. Rationale, design, and baseline findings from a randomized trial of collaborative care for chronic musculoskeletal pain in primary care. Pain Med. 2008, 9, 1050-1064. [CrossRef]

68. Bath, B.; Grona, S.L.; Milosavljevic, S.; Sari, N.; Imeah, B.; O'Connell, M.E. Advancing interprofessional primary health care services in rural settings for people with chronic low back disorders: Protocol of a community-based randomized controlled trial. JMIR Res. Protoc. 2016, 5, e212. [CrossRef] [PubMed]

69. Gustavsson, C.; Nordlander, J.; Söderlund, A. Activity and life-role targeting rehabilitation for persistent pain: Feasibility of an intervention in primary healthcare. Eur. J. Physiother. 2018, 20, 141-151. [CrossRef]

70. Calner, T.; Nordin, C.; Eriksson, M.; Nyberg, L.; Gard, G.; Michaelson, P. Effects of a self-guided, web-based activity programme for patients with persistent musculoskeletal pain in primary healthcare: A randomized controlled trial. Eur. J. Pain 2017, 21, 1110-1120. [CrossRef] [PubMed]

71. Chelimsky, T.C.; Fischer, R.L.; Levin, J.B.; Cheren, M.I.; Marsh, S.K.; Janata, J.W. The primary practice physician Program for Chronic Pain (C 4PCP): Outcomes of a primary physician-pain specialist collaboration for community-based training and support. Clin. J. Pain 2013, 29, 1036-1043. [CrossRef]

72. Dobscha, S.K.; Corson, K.; Perrin, N.A.; Hanson, G.C.; Leibowitz, R.Q.; Doak, M.N.; Dickinson, K.C.; Sullivan, M.D.; Gerrity, M.S. Collaborative care for chronic pain in primary care: A cluster randomized trial. J. Am. Med. Assoc. 2009, 301, $1242-1252$. [CrossRef]

73. Helminen, E.-E.; Sinikallio, S.H.; Valjakka, A.L.; Väisänen-Rouvali, R.H.; Arokoski, J.P.A. Effectiveness of a cognitive-behavioural group intervention for knee osteoarthritis pain: A randomized controlled trial. Clin. Rehabil. 2015, 29, 868-881. [CrossRef]

74. Nordin, C.A.; Michaelson, P.; Gard, G.; Eriksson, M.K.; Gustavsson, C.; Buhrman, M. Effects of the web behavior change program for activity and multimodal pain rehabilitation: Randomized controlled trial. J. Med. Internet Res. 2016, 18, e265. [CrossRef]

75. Hansson, E.E.; Sörensson, E.; Ronnheden, A.-M.; Lundgren, M.; Bjärnung, A.; Dahlberg, L.E. Arthritis school in primary health care. A pilot study of 14 years of experiences from Malmo. Lakartidningen 2008, 105, 2175-2177.

76. Sundberg, T.; Halpin, J.; Warenmark, A.; Falkenberg, T. Towards a model for integrative medicine in Swedish primary care. BMC Health Serv. Res. 2007, 7, 107. [CrossRef] 
77. DeBar, L.; Benes, L.; Bonifay, A.; Deyo, R.A.; Elder, C.R.; Keefe, F.J.; Leo, M.C.; McMullen, C.; Mayhew, M.; Owen-Smith, A.; et al. Interdisciplinary team-based care for patients with chronic pain on long-term opioid treatment in primary care (PPACT) - Protocol for a pragmatic cluster randomized trial. Contemp. Clin. Trials 2018, 67, 91-99. [CrossRef]

78. Helminen, E.-E.; Sinikallio, S.H.; Valjakka, A.L.; Väisänen-Rouvali, R.H.; Arokoski, J.P. Effectiveness of a cognitive-behavioral group intervention for knee osteoarthritis pain: Protocol of a randomized controlled trial. BMC Musculoskelet. Disord. 2013, 14, 46. [CrossRef]

79. Nordin, C.; Michaelson, P.; Eriksson, M.K.; Gard, G. It's about me: Patients' experiences of patient participation in the web behavior change program for activity in combination with multimodal pain rehabilitation. J. Med. Internet Res. 2017, 19, e22. [CrossRef]

80. Dunstan, D.A.; Covic, T. Can a rural community-based work-related activity program make a difference for chronic pain-disabled injured workers? Aust. J. Rural Health 2007, 15, 166-171. [CrossRef]

81. Dunstan, D.A. Participants' evaluation of a brief intervention for pain-related work disability. Int. J. Rehabil. Res. 2014, 37, 368-370. [CrossRef]

82. Gurden, M.; Morelli, M.; Sharp, G.; Baker, K.; Betts, N.; Bolton, J. Evaluation of a general practitioner referral service for manual treatment of back and neck pain. Prim. Health Care Res. Dev. 2012, 13, 204-210. [CrossRef]

83. Mårtensson, L.; Marklund, B.; Fridlund, B. Evaluation of a biopsychosocial rehabilitation programme in primary healthcare for chronic pain patients. Scand. J. Occup. Ther. 1999, 6, 157-165.

84. Mårtensson, L.; Marklund, B.; Baigi, A.; Gunnarsson, M.; Fridlund, B. Long-term influences of a biopsychosocial rehabilitation programme for chronic pain patients. Musculoskelet. Care 2004, 2, 152-164. [CrossRef]

85. Mårtensson, L.; Dahlin-Ivanoff, S. Experiences of a primary health care rehabilitation programme. A focus group study of persons with chronic pain. Disabil. Rehabil. 2006, 28, 985-995. [CrossRef]

86. Schütze, R.; Slater, H.; O'Sullivan, P.; Thornton, J.; Finlay-Jones, A.; Rees, C.S. Mindfulness-based functional therapy: A preliminary open trial of an integrated model of care for people with persistent low back pain. Front. Psychol. $2014,5,839$.

87. Stein, K.F.; Miclescu, A. Effectiveness of multidisciplinary rehabilitation treatment for patients with chronic pain in a primary health care unit. Scand. J. Pain 2013, 4, 190-197. [CrossRef]

88. Tyack, Z.; Frakes, K.-A.; Cornwell, P.; Kuys, S.S.; Barnett, A.G.; McPhail, S.M. The health outcomes and costs of people attending an interdisciplinary chronic disease service in regional Australia: Protocol for a longitudinal cohort investigation. BMC Health Serv. Res. 2013, 13, 410. [CrossRef]

89. Westman, A.; Linton, S.J.; Theorell, T.; Öhrvik, J.; Wåhlén, P.; Leppert, J. Quality of life and maintenance of improvements after early multimodal rehabilitation: A 5-year follow-up. Disabil. Rehabil. 2006, 28, 437-446. [CrossRef]

90. Westman, A.; Linton, S.J.; Öhrvik, J.; Wahlén, P.; Theorell, T.; Leppert, J. Controlled 3-year follow-up of a multidisciplinary pain rehabilitation program in primary health care. Disabil. Rehabil. 2009, 32, 307-316. [CrossRef]

91. Dorflinger, L.M.; Ruser, C.; Sellinger, J.; Edens, E.L.; Kerns, R.D.; Becker, W.C. integrating interdisciplinary pain management into primary care: Development and implementation of a novel clinical program. Pain Med. 2014, 15, 2046-2054. [CrossRef]

92. Lovo, S.; Harrison, L.; O'Connell, M.E.; Trask, C.; Bath, B. Experience of patients and practitioners with a team and technology approach to chronic back disorder management. J. Multidiscip. Health 2019, 12, 855-869. [CrossRef]

93. Holmner, E.P.; Stålnacke, B.-M.; Enthoven, P.; Stenberg, G. “The acceptance” of living with chronic pain-An ongoing process: A qualitative study of patient experiences of multimodal rehabilitation in primary care. J. Rehabil. Med. 2018, 50, 73-79. [CrossRef]

94. Stenberg, G.; Holmner, E.P.; Stålnacke, B.-M.; Enthoven, P. Healthcare professional experiences with patients who participate in multimodal pain rehabilitation in primary care-A qualitative study. Disabil. Rehabil. 2016, 38, 2085-2094. [CrossRef]

95. Sundberg, T.; Petzold, M.; Wändell, P.; Ryden, A.; Falkenberg, T. Exploring integrative medicine for back and neck pain-A pragmatic randomised clinical pilot trial. BMC Complement. Altern. Med. 2009, 9, 33. [CrossRef]

96. Quanjel, T.C.C.; Spreeuwenberg, M.D.; Struijs, J.N.; Baan, C.A.; Ruwaard, D. Substituting hospital-based outpatient cardiology care: The impact on quality, health and costs. PLoS ONE 2019, 14, e0217923. [CrossRef]

97. Sibbald, B.; Pickard, S.; McLeod, H.; Reeves, D.; Mead, N.; Gemmell, I.; Coast, J.; Roland, M.; Leese, B. Moving specialist care into the community: An initial evaluation. J. Health Serv. Res. Policy 2008, 13, 233-239. [CrossRef]

98. Nocon, A.; Rhodes, P.J.; Wright, J.P.; Eastham, J.; Williams, D.R.R.; Harrison, S.R.; Young, R.J. Specialist general practitioners and diabetes clinics in primary care: A qualitative and descriptive evaluation. Diabet. Med. 2004, 21, 32-38. [CrossRef] [PubMed]

99. Obucina, M.; Harris, N.; Fitzgerald, J.; Chai, A.; Radford, K.; Ross, A.; Carr, L.; Vecchio, N. The application of triple aim framework in the context of primary healthcare: A systematic literature review. Health Policy 2018, 122, 900-907. [CrossRef] [PubMed]

100. Kerman, N.; Kidd, S.A. The healthcare triple aim in the recovery era. Adm. Policy Ment. Health Ment. Health Serv. Res. 2020, 47, 492-496. [CrossRef] [PubMed]

101. Grant, M.; Joanne, O.; Froud, R.; Underwood, M.; Seers, K. The work of return to work. Challenges of returning to work when you have chronic pain: A meta-ethnography. Br. Med. J. Open 2019, 9, e025743. [CrossRef]

102. Furlan, A.D.; Zhao, J.; Voth, J.; Hassan, S.; Dubin, R.; Stinson, J.N.; Jaglal, S.; Fabico, R.; Smith, A.J.; Taenzer, P.; et al. Evaluation of an innovative tele-education intervention in chronic pain management for primary care clinicians practicing in underserved areas. J. Telemed. Telecare 2019, 25, 484-492. [CrossRef]

103. Skamagki, G.; King, A.; Duncan, M.; Wåhlin, C. A systematic review on workplace interventions to manage chronic musculoskeletal conditions. Physiother. Res. Int. 2018, 23, e1738. [CrossRef] 
104. Dubin, R.; King-VanVlack, C. The trajectory of chronic pain: Can a community-based exercise/education program soften the ride? Pain Res. Manag. 2010, 15, 361-368. [CrossRef]

105. World Health Organization. Integreated health services-What and why? In Technical Brief; WHO: Geneva, Switzerland, 2008.

106. Hoffmann, T.C.; Glasziou, P.P.; Boutron, I.; Milne, R.; Perera, R.; Moher, D.; Altman, D.G.; Barbour, V.; Macdonald, H.; Johnston, M.; et al. Better reporting of interventions: Template for intervention description and replication (TIDieR) checklist and guide. Br. Med. J. 2014, 348, 1687. [CrossRef] 